\title{
Spot Self-Replication and Dynamics for the Schnakenburg Model in a Two-Dimensional Domain
}

\author{
T. KOLOKOLNIKOV, M. J. WARD, and J. WEI \\ Theodore Kolokolnikov; Department of Mathematics, Dalhousie University, Halifax, Nova Scotia, B3H 3J5, Canada, \\ Michael Ward; Department of Mathematics, University of British Columbia, Vancouver, British Columbia, V6T 1Z2, Canada, \\ Juncheng Wei, Department of Mathematics, Chinese University of Hong Kong, Shatin, New Territories, Hong Kong.
}

(Received 10 November 200\%)

\begin{abstract}
The dynamical behavior of multi-spot solutions in a two-dimensional domain $\Omega$ is analyzed for the two-component Schnakenburg reaction-diffusion model in the singularly perturbed limit of small diffusivity $\varepsilon$ for one of the two components. In the limit $\varepsilon \rightarrow 0$, a quasi-equilibrium spot pattern in the region away from the spots is constructed by representing each localized spot as a logarithmic singularity of unknown strength $S_{j}$ for $j=1, \ldots, K$ at unknown spot locations $x_{j} \in \Omega$ for $j=1, \ldots, K$. A formal asymptotic analysis, which has the effect of summing infinite logarithmic series in powers of $-1 / \log \varepsilon$, is then used to derive an ODE differential algebraic system (DAE) for the collective coordinates $S_{j}$ and $x_{j}$ for $j=1, \ldots, K$, which characterizes the slow dynamics of a spot pattern. This DAE system involves the Neumann Green's function for the Laplacian. By numerically examining the stability thresholds for a single spot solution, a specific criterion in terms of the source strengths $S_{j}$, for $j=1, \ldots, K$, is then formulated to theoretically predict the initiation of a spot-splitting event. The analytical theory is illustrated for spot patterns in the unit disk and the unit square, and is compared with full numerical results computed directly from the Schnakenburg model.
\end{abstract}

Key words: singular perturbations, spots, self-replication, logarithmic expansions, Neumann Green's function, nonlocal eigenvalue problem.

\section{Introduction}

Localized spatio-temporal patterns consisting of spots or clusters of spots have been observed in many physical and chemical experiments. Such localized patterns can exhibit a variety of dynamical behaviors and instabilities including slow spot drift, temporal oscillations of spots, spot annihilation, and spot self-replication. Physical experiments where some of this phenomena has been observed include the ferrocyanide-iodate-sulphite reaction (cf. [28], the chloridedioxide-malonic acid reaction (cf. [11]), and certain semiconductor gas discharge systems (cf. [3], [4], [36]).

Numerical simulations of certain singularly perturbed two-component reaction-diffusion systems with very simple kinetics, such as the Gray-Scott model, have shown the occurrence of very complex spatio-temporal localized patterns consisting of either spots, stripes, or space-filling curves in a two-dimensional domain (cf. [39], [35], [27], [30]). Some of these reduced two-component reaction-diffusion systems model, at least qualitatively, the more complex chemically interacting systems of the experimental studies of [28] and [11]. Alternatively, three-component reaction diffusion systems (cf. [7]) have been used for modeling the dynamics and instabilities of spot patterns that have been observed in certain gas-discharge experiments (cf. [3], [4], [36]). A survey of experimental and theoretical studies, through reaction-diffusion modeling, of localized spot patterns in various physical or chemical contexts is given in [44].

Mathematically, a spot pattern for a reaction-diffusion system in a multi-dimensional domain $\Omega$ is a spatial pattern where at least one of the solution components is highly localized near certain discrete points in $\Omega$ that can evolve dynamically in time. For certain singularly perturbed two-component reaction-diffusion models in one space dimension, 
such as the Gray-Scott and Gierer-Meinhardt models, there has been considerable analytical progress in understanding both the dynamics and the various types of instabilities of spike patterns, including self-replicating instabilities (see $[\mathbf{3 7}],[\mathbf{3 8}],[\mathbf{4 3}],[\mathbf{1 5}],[\mathbf{1 2}],[\mathbf{2 5}],[\mathbf{4 1}]$ and many of the references therein). In contrast, in a two-dimensional spatial domain there are only a few analytical results characterizing spot dynamics, such as $[\mathbf{9}],[\mathbf{2 4}]$, and $[\mathbf{4 6}]$, for a one-spot solution of the Gierer-Meinhardt model, and the studies of $[\mathbf{1 6}],[\mathbf{1 7}]$, and $[\mathbf{1 8}]$, for exponentially weakly interacting spots in various contexts. Alternatively, for PDE models that admit a variational formulation, such as the Ginzburg-Landau type models of superconductivity, there are many formal asymptotic (cf. [14]) and rigorous (cf. [22]) results for the dynamics of localized vortices in two-dimensional domains. With regards to the stability of equilibrium multi-spot patterns for singularly perturbed reaction diffusion systems, an analytical theory based on the rigorous derivation and analysis of certain nonlocal eigenvalue problems (NLEP) has been developed in [48], [49], $[\mathbf{5 0}],[\mathbf{5 1}],[\mathbf{5 2}]$, and [53], for the Gierer-Meinhardt and Gray-Scott models. A survey of this theory, together with a further application of it to the Schnakenburg model, is given in [54].

The goal of this paper is to study the dynamics and instabilities of spot patterns for a certain limiting form of the singularly perturbed two-component Schnakenburg model

$$
\mathcal{V}_{t}=\varepsilon^{2} \Delta \mathcal{V}+b-\mathcal{V}+\mathcal{U} \mathcal{V}^{2}, \quad \mathcal{U}_{t}=D_{u} \Delta \mathcal{U}+a-\mathcal{U} \mathcal{V}^{2}, \quad x \in \Omega ; \quad \partial_{n} \mathcal{U}=\partial_{n} \mathcal{V}=0, \quad x \in \partial \Omega
$$

For this model, $\mathcal{V}$ is spatially localized and the full numerical computations of [31] have shown the occurrence of spot-splitting for $\mathcal{V}$ on a slowly growing time-dependent domain. In the simpler context of a one-dimensional domain, the stability problem for equilibrium spike patterns for (1.1) with $b=0$ has been studied analytically in $[\mathbf{2 3}]$ and [47]. Moreover, in certain parameter regimes it has been shown numerically in [5], [10], and [19], that spike patterns for (1.1) can undergo self-replication in a slowly growing one-dimensional domain.

To facilitate the analysis, in this paper we will consider (1.1) in the limit $\varepsilon \rightarrow 0$ with $D_{u}=D / \varepsilon^{2}$, where $D=\mathcal{O}(1)$. In this limit, we introduce the new variables $v$ and $u$ by $v=\varepsilon^{2} \mathcal{V}$ and $u=\varepsilon^{-2} \mathcal{U}$. Upon substituting these scalings into (1.1), and neglecting the asymptotically negligible $b \varepsilon^{2}$ term, we obtain the simplified system

$$
v_{t}=\varepsilon^{2} \Delta v-v+u v^{2}, \quad \varepsilon^{2} u_{t}=D \Delta u+a-\varepsilon^{-2} u v^{2}, \quad x \in \Omega ; \quad \partial_{n} u=\partial_{n} v=0, \quad x \in \partial \Omega .
$$

Here $0<\varepsilon \ll 1, D>0$, and $a>0$, are parameters. In this paper, we will also refer to (1.2) as the Schnakenburg model. This limiting system is similar to the Gray-Scott model, but its solution behavior is somewhat simpler.

The explicit goal of this paper is to develop a formal asymptotic analysis in the limit $\varepsilon \rightarrow 0$ to explicitly characterize the slow dynamics of quasi-equilibrium multi-spot patterns for (1.2). A combination of numerical and analytical techniques is then used to determine the stability of the quasi-equilibrium spot patterns and to make explicit predictions for the onset of any spot-splitting events.

In $\S 2.1$ we use the method of matched asymptotic expansions to construct a one-spot quasi-equlibrium solution to (1.2) centered at some $x=x_{0} \in \Omega$. This construction is done in terms of the solution $V(\rho)$ and $U(\rho)$, with $\rho \equiv \varepsilon^{-1}\left|x-x_{0}\right|$, to the following coupled nonlinear radially symmetric "core problem":

$$
\begin{aligned}
V_{\rho \rho}+\frac{1}{\rho} V_{\rho}-V+U V^{2} & =0, \quad U_{\rho \rho}+\frac{1}{\rho} U_{\rho}-U V^{2}=0, \quad 0<\rho<\infty, \\
V \rightarrow 0, \quad U & \sim S \log \rho+\chi(S)+O\left(\rho^{-1}\right), \quad \text { as } \quad \rho \rightarrow \infty .
\end{aligned}
$$

To construct a quasi-equilibrium one-spot pattern for (1.2), (1.3) is solved numerically for a range of source strength $S>0$, which then determines the function $\chi=\chi(S)$ in $(1.3 b)$. In the context of the Gray-Scott model in $\mathbb{R}^{2}$, 
this core problem, without the explicit far-field condition $(1.3 \mathrm{~b})$, was first identified in $\S 5$ of [34] and its solutions computed numerically. The far-field form $(1.3 b)$ for the (inner) core solution for $u$ then gives a Couloumb singularity $u \sim S \log \left|x-x_{0}\right|+S / \nu+\chi(S)$, where $\nu \equiv-1 / \log \varepsilon$, with a pre-specified non-singular part, for the corresponding outer solution for $u$ as $x \rightarrow x_{0}$. By analytically solving the outer problem for $u$ subject to this singularity structure, an algebraic equation for $S$ is derived that has the effect of summing all of the logarithmic terms in powers of $\nu$ involved in the determination of $S$. Related infinite logarithmic series in powers of $\nu$ also occur in the asymptotic analysis of various classes of linear and nonlinear eigenvalue problems and diffusion problems in two-dimensional domains that contain localized defects such as traps and holes (cf. $[\mathbf{8}],[\mathbf{4 0}],[\mathbf{4 2}],[\mathbf{4 5}])$. In contrast to the nonlinear core problem (1.3) involved in the analysis of (1.2), in all of these previous problems (cf. [8], [40], [42], [45]) the solution in the vicinity of the localized defect satisfies Laplace's equation, and hence the "inner", or local solution, can readily be found. In $\S 2.2$ we derive an explicit ODE for the dynamics of the spot location $x_{0}$ by first extending the asymptotic analysis of $\S 2.1$ in order to match transcendentally small $\mathcal{O}(\varepsilon)$ gradient terms in the inner and outer expansions of $u$, and then invoking a Fredholm solvability condition on a certain non self-adjoint linear operator. This analysis shows that the speed of the spot satisfies $x_{0}^{\prime}=\mathcal{O}\left(\varepsilon^{2}\right)$.

In $\S 2.3$ we numerically study the stability of a one-spot quasi-equilibrium solution to instabilities occuring on a fast $\mathcal{O}(1)$ time-scale relative to the slow spot dynamics of speed $\mathcal{O}\left(\varepsilon^{2}\right)$. Therefore, in the stability calculation we asymptotically freeze the spot location at some $x_{0} \in \Omega$. The perturbation in an $\mathcal{O}(\varepsilon)$ region near the spot is taken to have the angular dependence $e^{i m \theta}$, where $\theta=\arg (y)$ and $y=\varepsilon^{-1}\left(x-x_{0}\right)$. Potential instabilities on an $\mathcal{O}(1)$ time-scale are possible only with the integer angular modes $m=0,2,3, \ldots$, and not for the translation mode $m=1$. By numerically studing an eigenvalue problem associated with the linearization of the core problem, we show that each mode with $m \geq 2$ is unstable only when the source strength $S$ exceeds some critical value $\Sigma_{m}$. The ordering of these thresholds is such that $\Sigma_{2}<\Sigma_{3}<\Sigma_{4} \ldots$ Morevover, for values of $S$ on the range $0<S<\Sigma_{2}$, we show numerically that the one-spot quasi-equilibrium solution is stable to the $m=0$ mode corresponding to a locally radially symmetric perturbation. Therefore, as $S$ is increased, the dominant instability is to the peanut-splitting $m=2$ mode. This instability is found numerically to initiate a nonlinear spot-splitting event.

We remark that in the NLEP stability analyses for multi-spot patterns for the Gray-Scott, Gierer-Meinhardt, and Schnakenburg, models in $[\mathbf{4 8}],[\mathbf{4 9}],[\mathbf{5 0}],[\mathbf{5 1}],[\mathbf{5 2}],[\mathbf{5 3}]$, and [54], the scaling of parameters is such that the inner or "core problem" near each spot does not consist of a coupled system as in (1.3). Instead, to leading order in $\nu=-1 / \log \varepsilon$, in the inner region the fast variable is a multiple of the radially symmetric ground-state solution $w(\rho)$ satisfying $w^{\prime \prime}+\rho^{-1} w^{\prime}-w+w^{2}=0$, while the slow variable is locally constant. Therefore, for a perturbation with angular dependence $e^{i m \theta}$ with $m \geq 2$, the associated eigenvalue problem for $\Phi(\rho)$ is $\Phi^{\prime \prime}+\rho^{-1} \Phi^{\prime}-m^{2} \rho^{-2} \Phi-\Phi+2 w \Phi=$ $\lambda \Phi$ with $\Phi \rightarrow 0$ as $\rho \rightarrow \infty$. For this problem, $\operatorname{Re}(\lambda)<0$ for $m \geq 2$ (see Theorem 2.12 of [29]). Hence, when the core problem is determined by the scalar ground-state solution $w$ there is no peanut-splitting instability.

Although our asymptotic theory reliably predicts the onset of spot-splitting, the detailed nonlinear mechanism of this process is not well understood mathematically. However, in $\S 2.4$, we show how to determine the direction of spot-splitting relative to the direction of the spot motion for a one-spot quasi-equilibrium solution. This analysis, which takes into account the four-dimensional near zero eigenspace for $S$ near $\Sigma_{2}$ comprised of the two translational directions $\cos (\theta)$ and $\sin (\theta)$ together with the two independent peanut-splitting directions $\cos (2 \theta)$ and $\sin (2 \theta)$, shows that spot-splitting occurs in a direction perpendicular to the motion of the spot.

In $\S 3$ we extend the one-spot analysis of $\S 2$ to characterize the dynamics of a $K$-spot quasi-equilibrium solution 
with $K>1$. In the outer region, which is defined away from $\mathcal{O}(\varepsilon)$ neighborhoods of the spot locations, each spot for the outer solution for $u$ in (1.2) is represented as a logarithmic Coulomb singularity structure of unknown strength $S_{j}$, with a pre-specified non-singular part, at an instantaneous but unknown spot location $x_{j} \in \Omega$. By solving this outer problem for $u$ analytically, a nonlinear algebraic system for the source strengths $S_{j}$, for $j=1, \ldots, K$, is derived in terms of the $x_{j}$, for $j=1, \ldots, K$. Then, by matching $\mathcal{O}(\varepsilon)$ gradient terms in the inner and outer expansions of $u$, an ODE system for the slow dynamics of the spot locations $x_{j}$, with $x_{j}^{\prime}=\mathcal{O}\left(\varepsilon^{2}\right)$, is derived in terms of the source strengths $S_{j}$, for $j=1, \ldots, K$. The resulting differential algebraic system (DAE) for the collective coordinates $S_{j}$ and $x_{j}$, for $j=1 \ldots, K$, involves the Neumann Green's function for the Laplacian and its regular part, together with exactly two nonlinear functions of $S$ that must be computed from the nonlinear core problem.

In $\S 3.1$ we study the stability of a $K$-spot quasi-equilibrium solution to instabilities occuring on a fast $\mathcal{O}(1)$ timescale relative to the slow spot dynamics of speed $\mathcal{O}\left(\varepsilon^{2}\right)$. For non-radially symmetric perturbations near each spot of integer angular mode $m \geq 2$, we show that there is no effect due to inter-spot coupling and hence the $j^{\text {th }}$ spot is stable to these modes if and only if its source strength $S_{j}$ is below the peanut-instability threshold $\Sigma_{2} \approx 4.3$ for the $m=2$ mode associated with the one-spot solution of $\S 2$. In contrast, we show analytically that the stability problems near each spot for the locally radially symmetric $m=0$ mode must be coupled together through a global perturbation of the slow component $u$. This inter-spot coupling leads to a novel global matrix eigenvalue problem governing the stability of the $K$-spot pattern to the local $m=0$ modes. For $D=\mathcal{O}(1)$, and to leading order in $\nu=-1 / \log \varepsilon$ as $\nu \rightarrow 0$, we show that this global eigenvalue problem does not generate any instabilities.

For certain domains $\Omega$, in $\S 4$ we derive some explicit analytical formulae for the Neumann Green's function $G(x ; \xi)$ and its regular part $R(\xi ; \xi)$, defined by $R(\xi ; \xi) \equiv \lim _{x \rightarrow \xi}\left[G(x ; \xi)+\frac{1}{2 \pi} \log |x-\xi|\right]$. These formulae are required in order to numerically solve the asymptotic DAE system characterizing the dynamics of a $K$-spot solution for (1.2). When $\Omega$ is the unit disk, explicit and simple formulae for these functions are well-known (see [24] and [26]). However, such simple formulae are not readily available for a rectangular domain. For this case, starting from a very slowly converging Fourier series representation, we show how to represent $G(x ; \xi)$ and its regular part $R(\xi ; \xi)$ in terms of rapidly converging series that can readily be used in the asymptotic DAE system for the spot dynamics. Our method for obtaining this alternative improved representation is closely related to the well-known technique of Ewald summation for summing slowly converging series. It is also related to the method developed in $[\mathbf{3 2}]$ and $[\mathbf{3 3}]$ that was used recently in $[\mathbf{4 0}]$ and $[\mathbf{8}]$ to analyze some linear diffusion problems in perforated domains.

In a series of numerical experiments, in $\S 5$ we favorably compare results obtained from the asymptotic DAE system for the dynamics of $K$-spot quasi-equilibria, together with our predictions for the onset of spot-splitting instablities, with corresponding full numerical results computed from (1.2) when $\Omega$ is either a disk or a square domain. As an illustration of our results, let $\Omega=[0,1] \times[0,1]$ be the unit square and consider an initial six-spot pattern when $\varepsilon=0.02, a=51$, and $D=0.1$, in (1.2). The six spots are initially taken to be equi-distributed on a circle of radius $r_{c}=0.33$ centered at the midpoint $x_{c}=(0.5,0.5)$ of the square. The initial spot locations $x_{j}$ at $t=0$, for $j=1, \ldots, 6$, are labeled in a counterclockwise way starting from the spot in Fig. 1(a) with the largest horizontal cartesian coordinate. The initial source strengths from the asymptotic DAE system are computed as $S_{1}=S_{4} \approx 4.01$, and $S_{2}=S_{3}=S_{5}=S_{6} \approx 4.44$. Since $S_{j}>\Sigma_{2} \approx 4.3$ for $j=2,3,5,6$, the asymptotic theory predicts that these four spots will undergo a spot-splitting process beginning at $t=0$. This prediction is confirmed by the full numerical results shown in Fig. 1 as computed from (1.2). Moreover, as shown in Experiment 5 of $\S 5$, if we use the full numerical results to give initial values for the ten spot locations for the asymptotic DAE system at a time slightly after the spot 


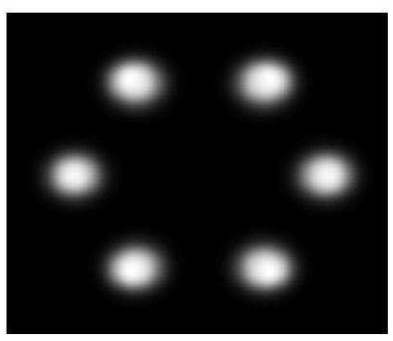

(a) $t=4.0$

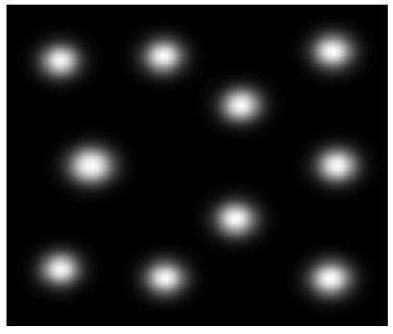

(d) $t=280.3$

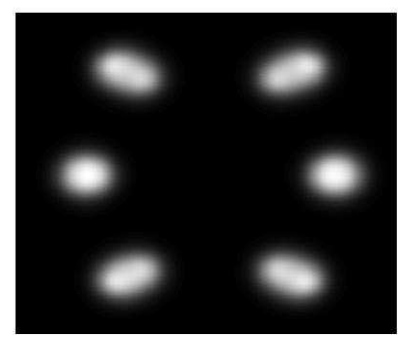

(b) $t=25.5$

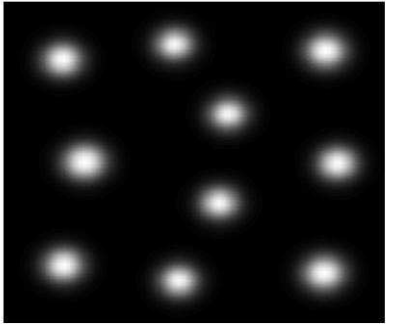

(e) $t=460.3$

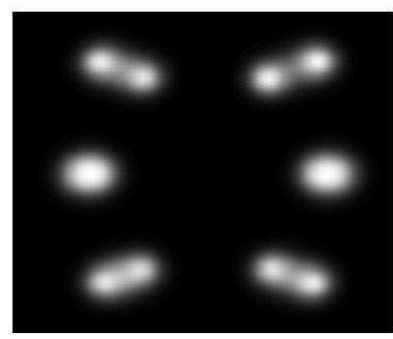

(c) $t=40.3$

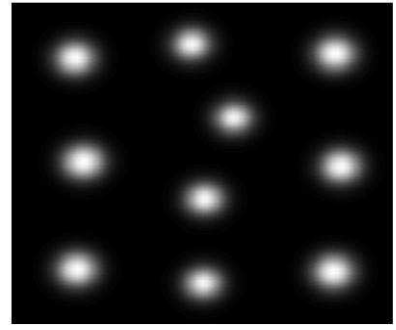

(f) $t=940.3$

Figure 1. Grayscale plot of $v$ computed from (1.2) in the unit square for the parameter values $\varepsilon=0.02, a=51, D=0.1$. The initial condition is a six-spot pattern with spots equi-distributed on a ring of radius $r_{c}=0.33$ centered at the midpoint of the square. Four of the initial spots undergo a splitting process, leading to a final ten-spot equilibrium pattern.

self-replication processes have terminated, then the asymptotic DAE system accurately predicts the spot trajectores at all later times, and in particular it predicts the final equilibrium state in Fig. 1(f) (see Fig. 12 below in $\S 5$ ).

When $\Omega$ is the unit disk, in $\S 5.1$ and $\S 5.2$ we show that the asymptotic DAE system for the spot dynamics can be studied analytically for two types of ring configurations of spots. For the case of $K>1$ spots equi-distributed on a ring, we derive a nonlinear first order ODE for the time-dependent ring radius that has a unique equilibrium point inside the disk. For this pattern, the spots have a common source strength $S_{c}=S_{j}$, for $j=1, \ldots, K$. We show numerically that all of the spots will split simulataneously if $S_{c}>\Sigma_{2} \approx 4.3$. Our second type of ring pattern in the unit disk involves $K-1$, with $K \geq 3$, spots equi-distributed on a ring together with one spot at the center of the disk. For this pattern, we show analytically how to construct a ring pattern that is initially stable to spot-splitting at time $t=0$ but that will become unstable to spot-splitting at a later time before the ring radius approaches its equilibrium value. This type of instability is referred to as a dynamically induced or triggered instability. Different types of dynamically induced instabilities are well-known to occur for spike patterns in one spatial dimension for the Gierer-Meinhardt and Gray-Scott models (see [41] and the references therein). This is the first illustration of such a phenomena in a two-dimensional domain.

In the asymptotic limit of large diffusivity $D=D_{0} / \nu \gg \mathcal{O}(1)$, where $\nu \equiv-1 / \log \varepsilon$ and $D_{0}=\mathcal{O}(1)$, in $\S 6$ we analyze the stability of a $K$-spot quasi-equilibrium pattern to a locally radially symmetric perturbation near each spot. For $D=\mathcal{O}\left(\nu^{-1}\right) \gg 1$ we show that each core solution for $v$ can be closely approximated by a scalar multiple 
of the radially-symmetric ground state solution $w(\rho)$ satisfying $w^{\prime \prime}+\rho^{-1} w-w+w^{2}=0$. Moreover, in the limit $D \gg 1$, we show that the stability problem of $\S 3.1$ for the $m=0$ mode reduces to the vectorial nonlocal eigenvalue problem (NLEP) of [54] that governs the stability of a $K$-spot quasi-equilibrium pattern to locally radially symmetric perturbations near each spot. The stability requirement of [54] that $D_{0}<D_{0 K}$, for some explicit threshold $D_{0 K}$, is then recovered. Finally, some open problems suggested by this study are listed in $\S 7$.

\section{One-Spot Solutions}

We first consider a one-spot solution to (1.2). We construct the quasi-equilibrium profile for the spot, we study its stability, and we derive an ODE for the center of the spot as it tends to its equilibrium location inside $\Omega$.

\subsection{A Quasi-Equilibrium One-Spot Solution}

We use matched asymptotic expansions to construct a quasi-equilibrium one-spot solution to (1.2) centered at some point $x_{0} \in \Omega$. The construction of such a solution consists of an outer region where $v$ is exponentially small and $u=\mathcal{O}(1)$, and an inner region of extent $\mathcal{O}(\varepsilon)$ centered at $x_{0}$ where both $v$ and $u$ have sharp gradients.

In the inner region we introduce new variables $\mathcal{V}(y)$ and $\mathcal{U}(y)$ by

$$
u=\frac{1}{\sqrt{D}} \mathcal{U}, \quad v=\sqrt{D} \mathcal{V}, \quad y=\varepsilon^{-1}\left(x-x_{0}\right) .
$$

Let $\Delta_{y}$ denote the Laplacian in $y$. Then, substituting (2.1) into the steady-state equations of (1.2) we get

$$
\Delta_{y} \mathcal{V}-\mathcal{V}+\mathcal{U} \mathcal{V}^{2}=0, \quad \Delta_{y} \mathcal{U}+\frac{a \varepsilon^{2}}{\sqrt{D}}-\mathcal{U} \mathcal{V}^{2}=0, \quad y \in \mathbb{R}^{2}
$$

To leading order, we look for a radially symmetric solution to $(2.2)$ given by $\mathcal{U} \sim U(\rho)$ and $\mathcal{V} \sim V(\rho)$ with $\rho=|y|$. Therefore, $U$ and $V$ satisfy

$$
V_{\rho \rho}+\frac{1}{\rho} V_{\rho}-V+U V^{2}=0, \quad U_{\rho \rho}+\frac{1}{\rho} U_{\rho}-U V^{2}=0, \quad 0<\rho<\infty .
$$

In order to match this solution to the outer solution constructed below, we require that $U$ grows logarithmically as $\rho \rightarrow \infty$. Therefore, we will solve $(2.3 a)$ subject to $U^{\prime}(0)=V^{\prime}(0)=0$ and the far-field condition

$$
V \rightarrow 0, \quad U \sim S \log \rho+\chi(S)+O\left(\rho^{-1}\right), \quad \text { as } \quad \rho \rightarrow \infty .
$$

We refer to (2.3) as the core problem. The Divergence theorem on (2.3) yields that $S=\int_{0}^{\infty} \rho U V^{2} d \rho>0$. We solve (2.3) numerically using COLSYS [2] for a range of values of the constant $S$. In terms of this solution, at each $S$ we numerically compute the constant $\chi(S)$ in $(2.3 b)$. In Fig. 2 we plot $U(0), 10 V(0)$, and $\chi$, versus $S$. In addition, we plot the solution $V(\rho)$ and $U(\rho)$ for several values of $S$. For $S>S_{v} \approx 4.78$ our computations show that $V(\rho)$ has a volcano shape, where the maximum of $V$ occurs at some interior value $\rho>0$. The two-dimensional core problem (2.3) was first identified and its solutions computed numerically in $\S 5$ of [34]. The one-dimensional version of (2.3) plays a central role in understanding pulse-splitting for the Gray-Scott model in one spatial dimension (cf. [12], [34], [25]). A detailed bifurcation analysis of the one-dimensional core problem was given in [13].

Next, we determine the unknown source strength $S$ for the core problem by matching the far-field behavior of the core solution to an outer solution for $u$ valid away from $\mathcal{O}(\varepsilon)$ distances from $x_{0}$. In the outer region, $v$ is exponentially 


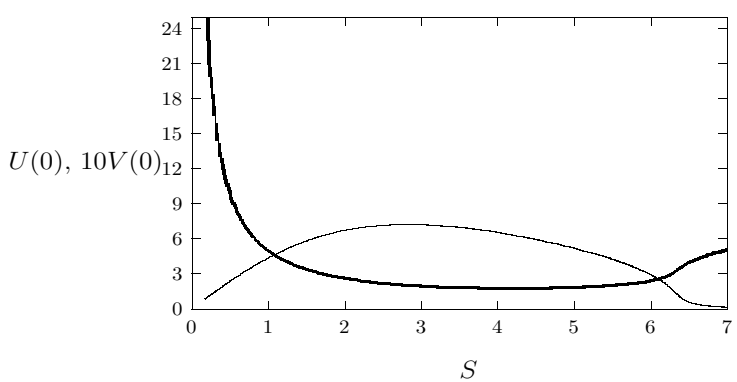

(a) $U(0)$ and $10 V(0)$ vs. $S$

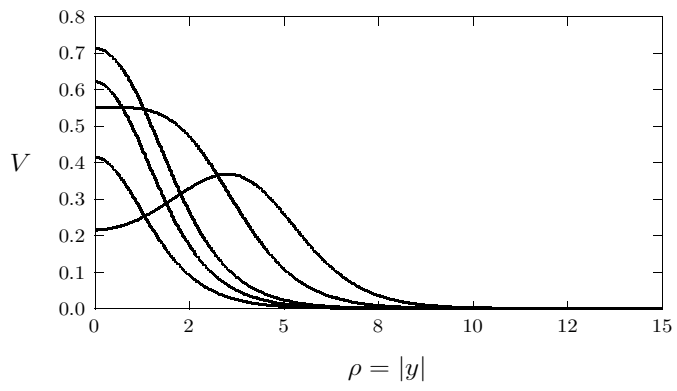

(c) $V$ vs. $\rho$

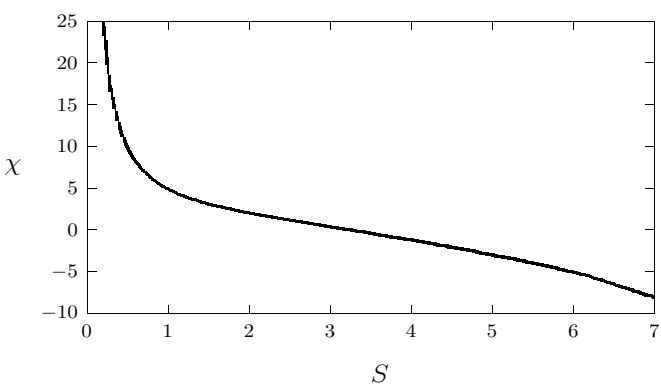

(b) $\chi$ vs. $S$

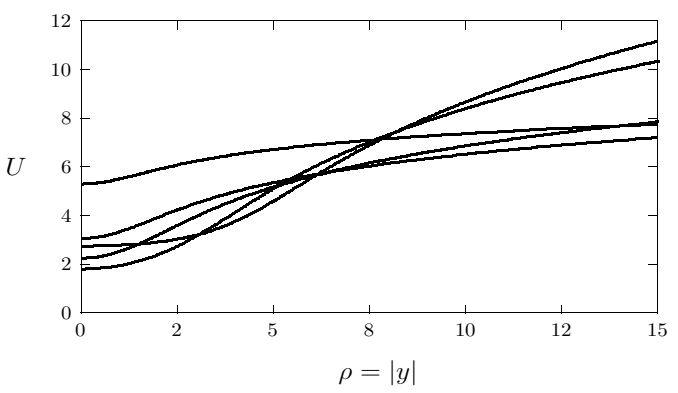

(d) $U$ vs. $\rho$

Figure 2. Numerical results computed from the core problem (2.3). Top left: $U(0)$ (heavy solid curve) and 10V(0) (solid curve) vs. $S$. Top right: $\chi$ vs. $S$. Bottom Row: $V(\rho)$ (left) and $U(\rho)$ (right) for $S=0.94, S=1.68, S=2.44, S=4.79$, and $S=6.19$. The specific labels of these curves correspond to the values of $U(0)$ and $10 V(0)$ in the top right figure. Notice that the profile for $V$ has a volcano shape when $S>S_{v} \approx 4.78$.

small, and from $(2.1)$ and $(2.3 b)$ we get

$$
\varepsilon^{-2} u v^{2} \rightarrow \frac{2 \pi \sqrt{D}}{\varepsilon^{2}}\left(\varepsilon^{2} \int_{0}^{\infty} \rho U V^{2} d \rho\right) \delta\left(x-x_{0}\right)=2 \pi \sqrt{D} S \delta\left(x-x_{0}\right) .
$$

Therefore, from (1.2), the outer steady-state solution for $u$ is

$$
\Delta u=-\frac{a}{D}+\frac{2 \pi}{\sqrt{D}} S \delta\left(x-x_{0}\right), \quad x \in \Omega ; \quad \partial_{n} u=0, \quad x \in \partial \Omega .
$$

The matching condition is that the near-field behavior of $u$ agrees with the far-field form of the core solution given by $u \sim D^{-1 / 2}[S \log |y|+\chi(S)]$, where $y=\varepsilon^{-1}\left(x-x_{0}\right)$. This matching condition yields

$$
u \sim \frac{1}{\sqrt{D}}\left[S \log \left|x-x_{0}\right|+\chi(S)+\frac{S}{\nu}\right] \quad \text { as } \quad x \rightarrow x_{0}, \quad \nu \equiv-1 / \log \varepsilon .
$$

The specification of a precise expression for the regular part of the singularity structure in $(2.5 b)$ for $u$ is the condition that yields a unique outer solution $u$. By using the Divergence theorem on $(2.5 a)$ we calculate $S$ as

$$
S=\frac{a|\Omega|}{2 \pi \sqrt{D}},
$$

where $|\Omega|$ denotes the area of $\Omega$. In order to conveniently represent the outer solution satisfying (2.5) we introduce 
the unique Neumann (or modified) Green's function $G\left(x ; x_{0}\right)$ and its regular part $R\left(x ; x_{0}\right)$ satisfying

$$
\begin{aligned}
& \Delta G=\frac{1}{|\Omega|}-\delta\left(x-x_{0}\right), \quad x \in \Omega ; \quad \partial_{n} G=0 \quad \text { on } \quad \partial \Omega, \\
& G\left(x ; x_{0}\right)=-\frac{1}{2 \pi} \log \left|x-x_{0}\right|+R\left(x ; x_{0}\right) ; \quad \int_{\Omega} G d x=0 .
\end{aligned}
$$

The self-interaction term $R_{0,0}$ is defined by $R_{0,0} \equiv R\left(x_{0} ; x_{0}\right)$. In $\S 4$ we analytically calculate $G\left(x ; x_{0}\right)$ and its regular part $R\left(x_{0} ; x_{0}\right)$ for either a disk or a rectangular domain.

The solution to $(2.5)$ is readily calculated as

$$
u(x)=-\frac{2 \pi}{\sqrt{D}}\left(S G\left(x ; x_{0}\right)+u_{c}\right),
$$

in terms of an as yet unknown constant $u_{c}$. We then use (2.7b) to expand $u$ as $x \rightarrow x_{0}$. Upon comparing the resulting expression with $(2.5 b)$, we determine $u_{c}$ in terms of $S$ as

$$
S+2 \pi \nu S R\left(x_{0} ; x_{0}\right)+\nu \chi(S)=-2 \pi \nu u_{c}, \quad \nu \equiv-1 / \log \varepsilon .
$$

Equation (2.6) determines $S$, and $u_{c}$ is determined in terms of $S$ by (2.9). With $S$ known, the core solution for $U$ and $V$ in an $\mathcal{O}(\varepsilon)$ neighborhood of the spot is given by

$$
u \sim \frac{1}{\sqrt{D}} U(\rho), \quad v \sim \sqrt{D} V(\rho),
$$

where $U$ and $V$ satisfy (2.3) with $S$ as given in (2.6). The outer solution for $u$, valid for $\left|x-x_{0}\right| \gg \mathcal{O}(\varepsilon)$, is given by (2.8). This completes the construction of a one-spot quasi-equilibrium solution.

\subsection{The Slow Dynamics of a One-Spot Solution}

We now derive an ODE for the slow dynamics of a one-spot solution. In the inner region near $x=x_{0}$ we expand

$$
u=\frac{1}{\sqrt{D}}\left(U(\rho)+\varepsilon U_{1}(y)+\cdots\right), \quad v=\sqrt{D}\left(V(\rho)+\varepsilon V_{1}(y)+\cdots\right), \quad y=\varepsilon^{-1}\left[x-x_{0}(\tau)\right], \quad \tau=\varepsilon^{2} t .
$$

Here $U(\rho)$ and $V(\rho)$, with $\rho=|y|$ are the radial symmetric solutions of the core problem (2.3) with $S$ as given in (2.6). By substituting (2.11) into (1.2), and collecting terms of order $\mathcal{O}(\varepsilon)$, we derive that $V_{1}$ and $U_{1}$ satisfy

$$
\Delta_{y} W_{1}+\mathcal{M}_{0} W_{1}=f, \quad y \in \mathbb{R}^{2},
$$

where the vectors $W_{1}, f$, and the $2 \times 2$ matrix $\mathcal{M}_{0}$, are defined by

$$
W_{1} \equiv\left(\begin{array}{c}
V_{1} \\
U_{1}
\end{array}\right), \quad f \equiv\left(\begin{array}{c}
-V^{\prime} x_{0}^{\prime} \cdot e_{\theta} \\
0
\end{array}\right), \quad e_{\theta} \equiv\left(\begin{array}{c}
\cos \theta \\
\sin \theta
\end{array}\right), \quad \mathcal{M}_{0} \equiv\left(\begin{array}{cc}
-1+2 U V & V^{2} \\
-2 U V & -V^{2}
\end{array}\right) .
$$

In the definition of $f, \cdot$ denotes the dot product.

The determination of an appropriate far-field condition for $W_{1}$ requires a higher order matching of the inner and outer solution for $u$. To do so, we expand the outer solution in (2.8) to include the gradient term by using

$$
G\left(x ; x_{0}\right) \sim-\frac{1}{2 \pi} \log \left|x-x_{0}\right|+R\left(x_{0} ; x_{0}\right)+\nabla R\left(x_{0} ; x_{0}\right) \cdot\left(x-x_{0}\right)+\cdots, \quad \text { as } \quad x \rightarrow x_{0} .
$$

Then, the matching condition for the inner and outer solutions for $u$ becomes

$$
U(\rho)+\varepsilon U_{1}(y)+\cdots \sim S \log \left|x-x_{0}\right|-2 \pi\left(S R\left(x_{0} ; x_{0}\right)+u_{c}\right)-2 \pi S \varepsilon \nabla R\left(x_{0} ; x_{0}\right) \cdot y+\cdots,
$$

where $U \sim S \log \rho+\chi(S)$ as $\rho=\varepsilon^{-1}\left|x-x_{0}\right| \rightarrow \infty$. Upon matching the gradient term in (2.14) with $U_{1}$, we conclude 
that $U_{1} \sim-2 \pi S \nabla R\left(x_{0} ; x_{0}\right) \cdot y$ as $y \rightarrow \infty$. Therefore, the solution to (2.12) must satisfy the far-field behavior

$$
W_{1} \sim\left(\begin{array}{c}
0 \\
\alpha \cdot y
\end{array}\right) \quad \text { as } y \rightarrow \infty, \quad \alpha \equiv-2 \pi S \nabla R\left(x_{0} ; x_{0}\right) .
$$

The problem (2.12) subject to (2.15) determines $x_{0}^{\prime}$ in terms of the vector $\alpha$. The result is written as follows:

Principal Result 2.1: Let $S$ be given as in (2.6). Then, a necessary condition for the existence of a solution of (2.12), subject to the far-field condition (2.15), is that

$$
x_{0}^{\prime}=\gamma(S) \alpha, \quad \gamma \equiv \gamma(S)=\frac{-2}{\int_{0}^{\infty} \rho V^{\prime}(\rho) \hat{\Phi}^{*}(\rho) d \rho} .
$$

Here $V(\rho)$ satisfies the core problem (2.3) at the given value of $S$, and $\hat{\Phi}^{*}(\rho)$ is the first component of the radially symmetric adjoint solution $\hat{P}^{*}(\rho) \equiv\left(\hat{\Phi}^{*}(\rho), \hat{\Psi}^{*}(\rho)\right)^{t}$ satisfying

$$
\Delta_{\rho} \hat{P}^{*}+\mathcal{M}_{0}^{t} \hat{P}^{*}=\mathbf{0}, \quad 0<\rho<\infty,
$$

subject to the far-field conditions that $\hat{\Phi}^{*} \rightarrow 0$ exponentially as $\rho \rightarrow \infty$ and that $\hat{\Psi}^{*} \sim 1 / \rho$ as $\rho \rightarrow \infty$. Here $\mathcal{M}_{0}^{t}$ denotes the transpose of the matrix $\mathcal{M}_{0}$ in (2.12b) and $\Delta_{\rho} \hat{P}^{*} \equiv \partial_{\rho \rho} \hat{P}^{*}+\rho^{-1} \partial_{\rho} \hat{P}^{*}-\rho^{-2} \hat{P}^{*}$.

We now derive this result. We begin by writing the homogeneous adjoint problem to $(2.12 a)$ as

$$
\Delta_{y} P^{*}+\mathcal{M}_{0}^{t} P^{*}=0, \quad y \in \mathbb{R}^{2}, \quad P^{*} \equiv\left(\begin{array}{c}
\Phi^{*} \\
\Psi^{*}
\end{array}\right) .
$$

We seek solutions to this problem as either $P_{c}^{*} \equiv \hat{P}^{*} \cos \theta$ or $P_{s}^{*} \equiv \hat{P}^{*} \sin \theta$, where $\hat{P}^{*}$ satisfies the radially symmetric problem (2.17). We write the two-component vector $\hat{P}^{*}$ as $\hat{P}^{*}=\left(\hat{\Phi}^{*}, \hat{\Psi}^{*}\right)$ and we impose the asymptotic boundary conditions $\hat{\Phi}^{*} \rightarrow 0$ exponentially as $\rho \rightarrow \infty$ and $\hat{\Psi}^{*} \sim \rho^{-1}$ as $\rho \rightarrow \infty$.

Next, we apply a solvability condition to the solution of (2.12) with (2.15) by applying Green's identity over a large ball $B_{\sigma}$ of radius $\sigma \gg 1$ centered at $y=0$. Upon using Green's identity to $P_{c}^{*}$ and $W_{1}$ we derive

$$
\begin{aligned}
\lim _{\sigma \rightarrow \infty} \int_{B_{\sigma}}\left[\left(P_{c}^{*}\right)^{t}\left(\Delta_{y} W_{1}+\mathcal{M}_{0} W_{1}\right)-\left(W_{1}\right)^{t}\left(\Delta_{y} P_{c}^{*}+\mathcal{M}_{0}^{t} P_{c}^{*}\right)\right] d y & \\
& =\left.\lim _{\sigma \rightarrow \infty} \int_{\partial B_{\sigma}}\left[\left(P_{c}^{*}\right)^{t} \partial_{\rho} W_{1}-W_{1}^{t} \partial_{\rho} P_{c}^{*}\right]\right|_{\rho=\sigma} d y .
\end{aligned}
$$

Then, upon using (2.12), together with the asymptotic boundary conditions for $W_{1}$ in $(2.15)$ and for $P_{c}^{*}$, we obtain that (2.19) reduces to

$$
-x_{01}^{\prime} \int_{0}^{2 \pi} \int_{0}^{\infty} \hat{\Phi}^{*} V^{\prime} \cos ^{2} \theta \rho d \rho d \theta=\left.\lim _{\sigma \rightarrow \infty} \int_{0}^{2 \pi}\left(\left(\frac{\cos \theta}{\rho}\right) \alpha_{1} \cos \theta-\alpha_{1} \rho \cos \theta\left(\frac{-1}{\rho^{2}}\right) \cos \theta\right)\right|_{\rho=\sigma} \sigma d \theta,
$$

where $x_{01}$ and $\alpha_{1}$ are the first components of $x_{0}$ and $\alpha$, respectively. Therefore, $x_{01}^{\prime} \int_{0}^{\infty} \rho V^{\prime} \hat{\Phi}^{*} d \rho=-2 \alpha_{1}$, which is the first component of (2.16). The second component of (2.16) follows by repeating this calculation with $P_{s}^{*}$.

By using (2.15) for $\alpha$ and recalling $\tau=\varepsilon^{2} t$, the slow dynamics of a one-spot solution satisfies the gradient flow

$$
\frac{d x_{0}}{d t} \sim-2 \pi \varepsilon^{2} \gamma(S) S \nabla R\left(x_{0} ; x_{0}\right) .
$$

The constant $\gamma=\gamma(S)$, defined in (2.16), must be computed numerically by first solving the core problem (2.3) and then computing the adjoint solution in (2.17). We plot $\gamma(S)$ in Fig. 3(a), and in Fig. 3(b) we plot the solution to the adjoint problem (2.17) when $S=3.51$. Our numerical computations show that $\gamma(S)>0$. Therefore, a stable equilibrium solution for (2.21) occurs at a minimum point of $R\left(x_{0} ; x_{0}\right)$. Since $S$ is independent of $x_{0}$ from (2.6), the constant multiplying $\nabla R\left(x_{0} ; x_{0}\right)$ in $(2.21)$ is independent of $x_{0}$. 


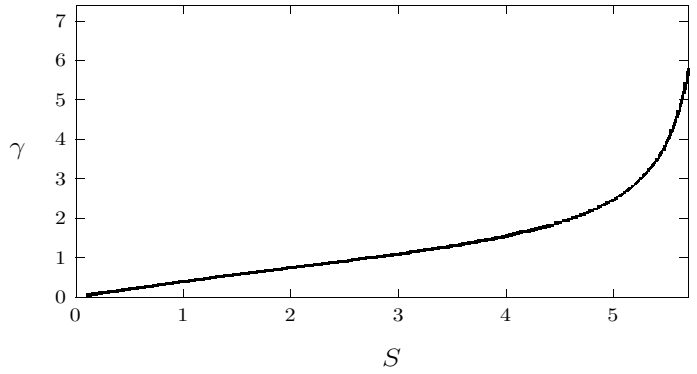

(a) $\gamma$ vs. $S$

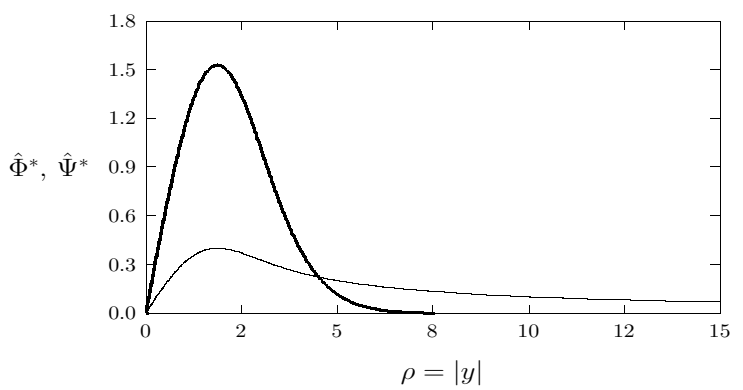

(b) $\hat{\Phi}^{*}$ and $\hat{\Psi}^{*}$ vs. $\rho=|y|$

Figure 3. Left figure: Numerical results for $\gamma(S)$ defined in (2.16). Right figure: the numerical solution $\hat{\Phi}^{*}(\rho)$ (heavy solid curve) and $\hat{\Psi}^{*}(\rho)$ (solid curve) to the adjoint problem (2.17) when $S=3.51$.

\subsection{The Stability of a One-Spot Solution}

Next, we study the stability of the quasi-equilibrium one-spot solution constructed above to instabilities occurring on a fast $\mathcal{O}(1)$ time-scale. Since the speed of the slow drift of the one-spot solution in $(2.21)$ is $\mathcal{O}\left(\varepsilon^{2}\right) \ll 1$, in our stability analysis we will assume that the spot is asymptotically stationary. We begin the stability analysis by letting $u_{e}$ and $v_{e}$ denote the quasi-equilibrium solution, and we introduce the perturbation

$$
u=u_{e}+\mathrm{e}^{\lambda t} \eta, \quad v=v_{e}+\mathrm{e}^{\lambda t} \phi .
$$

By substituting (2.22) into (1.2) and linearizing, we obtain the following eigenvalue problem for $\phi$ and $\eta$ :

$$
\varepsilon^{2} \Delta \phi-\phi+2 u_{e} v_{e} \phi+v_{e}^{2} \eta=\lambda \phi, \quad D \Delta \eta-2 \varepsilon^{-2} u_{e} v_{e} \phi-\varepsilon^{-2} v_{e}^{2} \eta=\varepsilon^{2} \lambda \eta, \quad x \in \Omega ; \quad \partial_{n} \phi=\partial_{n} \eta=0, \quad x \in \partial \Omega .
$$

In the inner region near $x_{0}$ we look for an $\mathcal{O}(1)$ time-scale instability associated with the local angular integer mode $m$ by introducing the new variables $N(\rho)$ and $\Phi(\rho)$ by

$$
\eta=\frac{1}{D} e^{i m \theta} N(\rho), \quad \phi=e^{i m \theta} \Phi(\rho), \quad \rho=|y|, \quad y=\varepsilon^{-1}\left(x-x_{0}\right),
$$

where $y^{t}=\rho(\cos \theta, \sin \theta)$. Substituting (2.24) into (2.23), and by using $u_{e} \sim D^{-1 / 2} U(\rho)$ and $v_{e} \sim \sqrt{D} V(\rho)$, where $U$ and $V$ satisfy the core problem (2.3), we obtain the following radially symmetric eigenvalue problem:

$$
\mathcal{L}_{m} \Phi-\Phi+2 U V \Phi+V^{2} N=\lambda \Phi, \quad \mathcal{L}_{m} N-2 U V \Phi-V^{2} N=0, \quad 0 \leq \rho<\infty .
$$

Here $\mathcal{L}_{m} \Phi \equiv \partial_{\rho \rho} \Phi+\rho^{-1} \partial_{\rho} \Phi-m^{2} \rho^{-2} \Phi$. We impose the usual regularity condition for $\Phi$ and $N$ at $\rho=0$. As we show below, the appropriate far-field boundary conditions for (2.25) as $\rho \rightarrow \infty$ depends on whether $m=0$ or $m \geq 2$.

The eigenvalue problem (2.25) does not appear to be amenable to analysis, and thus we solve it numerically for various integer values of $m$. We denote $\lambda_{0}$ to be the eigenvalue of (2.25) with the largest real part. Since $U$ and $V$ depend on $S$ from (2.3), we have implicitly that $\lambda_{0}=\lambda_{0}(S, m)$. To determine the onset of any instabilities, we compute any threshold values $S=\Sigma_{m}$ where $\operatorname{Re}\left(\lambda_{0}\left(\Sigma_{m}, m\right)\right)=0$. In our computations, we only consider $m=0,2,3,4, \ldots$, since $\lambda_{0}=0$ for any value of $S$ for the translational mode $m=1$. A higher order perturbation analysis for the $m=1$ mode generates only weak instabilities occurring on an asymptotically long $\mathcal{O}\left(\varepsilon^{-2}\right)$ time-scale. Any such instabilities are reflected in instabilities in the ODE (2.21). We consider the cases $m=0$ or $m \geq 2$ separately.

When $m \geq 2$ we can impose the asymptotic decay conditions that $\Phi$ decays exponentially as $\rho \rightarrow \infty$ while 


$$
|m| \quad \Sigma_{m} \mid
$$

$\left|\begin{array}{l|l}2 & 4.303 \\ 3 & 5.439 \\ 4 & 6.143 \\ 5 & 6.403 \\ 6 & 6.517\end{array}\right|$

Table 1. Numerical results computed from (2.25) for the threshold values of $S$, denoted by $\Sigma_{m}$, as a function of the integer angular mode $m$ where an instability first occurs for the core problem (2.3) as $S$ increases.

$N \sim \mathcal{O}\left(\rho^{-m}\right) \rightarrow 0$ as $\rho \rightarrow \infty$. With these conditions (2.25) is discretized with centered differences on a large but finite domain. We then determine $\lambda_{0}(S, m)$ by computing the eigenvalues of a matrix eigenvalue problem using LAPACK (cf. [1]). For $m \geq 2$ our computations show that $\lambda_{0}(S, m)$ is real and that $\lambda_{0}(S, m)>0$ when $S>\Sigma_{m}$. The threshold value $\Sigma_{m}$ is tabulated in Table 1 for $m=2, \ldots, 6$. In our computations we took 300 meshpoints on the interval $0 \leq \rho<20$. To the number of significant digits shown in Table 1, the results there are insensitive to increasing either the domain length or the number of grid points. It follows from Table 1 that the smallest value of $S$ where an instability is triggered occurs for the "peanut-splitting" instability $m=2$ at the threshold value $S=\Sigma_{2} \approx 4.3$. In Fig. 4(a) we plot $\lambda_{0}(S, m)$ as a function of $S$ for $m=2, m=3$ and $m=4$.

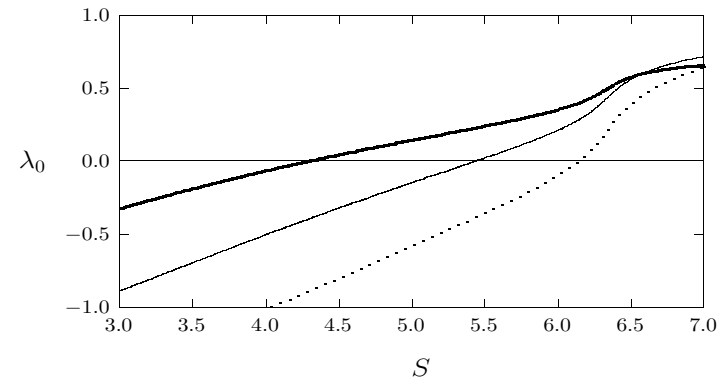

(a) $\lambda_{0}$ vs. $S$ for $m=2,3,4$

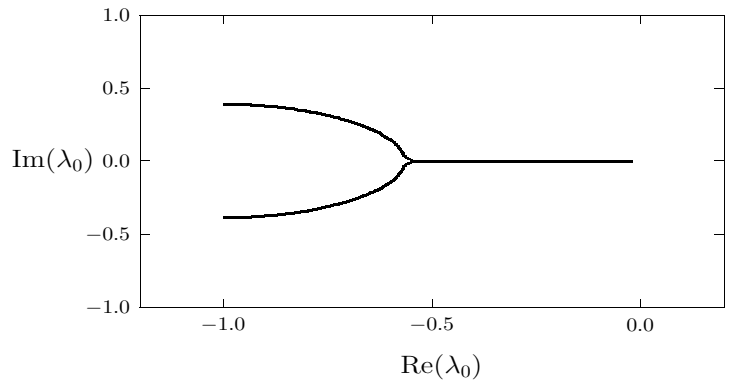

(b) $\operatorname{Im}\left(\lambda_{0}\right)$ and $\operatorname{Re}\left(\lambda_{0}\right)$ for $m=0$

Figure 4. Left figure: Plot of the largest (real) eigenvalue $\lambda_{0}(S, m)$ of (2.25) vs. $S$ for $m=2$ (heavy solid), $m=3$ (solid), and $m=4$ (dotted). Right figure: Plot in the complex plane of the path of the eigenvalue $\lambda_{0}(S, 0)$ of largest real part of $(2.25)$ with $m=0$ and $2.8<S<7.5$. For $S<2.8, \lambda_{0} \approx-1.0$ and arises from the discretization of the continuous spectrum (not shown). For $2.8<S<4.98, \lambda_{0}(S, 0)$ occurs as a complex conjugate pair which monotonically approaches the real axis as $S$ increases. This pair merges onto the real axis at $S \approx 4.79$. As $S$ increases further, $\lambda_{0}(S, 0)$ remains real but negative.

Next, we treat the case $m=0$. For this mode, $\Phi$ in (2.25) still decays exponentially as $\rho \rightarrow \infty$. However, we cannot apriori impose that $N$ in (2.25) is bounded as $\rho \rightarrow \infty$. Instead we must allow for the possibility of a logarithmic growth with $N(\rho) \sim C \log \rho$ as $\rho \rightarrow \infty$. The Divergence theorem on $\mathcal{L}_{0}$ in (2.25) identifies $C$ as

$$
C=\int_{0}^{\infty}\left(2 U V \Phi+N V^{2}\right) \rho d \rho .
$$

The constant $C$ will be determined by matching $N$ to an outer eigenfunction $\eta$, valid away from $x_{0}$, that satisfies (2.23). For this outer solution, since $v_{e}$ is localized near $x_{0}$, we can calculate in the sense of distributions that

$$
2 \varepsilon^{-2} u_{e} v_{e} \phi+\varepsilon^{-2} \eta v_{e}^{2} \rightarrow\left(\int_{\mathbb{R}^{2}}\left(2 U V \Phi+N V^{2}\right) d y\right) \delta\left(x-x_{0}\right)=2 \pi C D \delta\left(x-x_{0}\right) .
$$


By using this expression in (2.23), we obtain that $\eta$ satisfies

$$
\Delta \eta=2 \pi C \delta\left(x-x_{0}\right), \quad x \in \Omega ; \quad \partial_{n} \eta=0, \quad x \in \partial \Omega ; \quad \eta \sim C \log \left|x-x_{0}\right| \quad \text { as } \quad x \rightarrow x_{0} .
$$

From applying the Divergence theorem on (2.28) we conclude that $C=0$. Therefore, in numerically computing $\lambda_{0}(S, 0)$ for the $m=0$ mode from (2.25) we must impose that $N$ is bounded as $\rho \rightarrow \infty$. In Fig. 4(b) we plot the path of $\lambda_{0}(S, 0)$ in the complex plane showing that $\lambda_{0}(S, 0)$ remains in the left half-plane until at least $S<7.5$.

\subsection{The Direction of Splitting}

We now determine the direction of splitting relative to the direction of spot motion when $S \approx \Sigma_{2}$, so that the eigenvalue for the $m=2$ mode associated with the peanut-splitting instability is nearly zero. Recall that the two translational eigenvalues, corresponding to the $m=1$ mode, are always zero for the infinite-domain core eigenvalue problem (2.25). Therefore, for $S \approx \Sigma_{2}$, there are four near-zero eigenvalues in the spectrum of the linearization of the core solution: two corresponding to translations and two corresponding to splitting. By deriving a certain solvability condition for the quasi-stationary spot solution centered at $x_{0}$, we will determine the direction of splitting relative to spot motion.

Recall that the quasi-equilibrium core solution is constructed from the asymptotic expansion

$$
u=\frac{1}{\sqrt{D}} \mathcal{U}(y), \quad v=\sqrt{D} \mathcal{V}(y), \quad y=\varepsilon^{-1}\left[x-x_{0}(\tau)\right], \quad \tau=\varepsilon^{2} t,
$$

where, for $\rho=|y|$,

$$
\mathcal{U}=U(\rho)+\varepsilon U_{1}(y)+\cdots, \quad \mathcal{V}=V(\rho)+\varepsilon V_{1}(y)+\cdots .
$$

Here $U, V$ are the radially symmetric solutions of the core problem $(2.3 a)$, while $U_{1}, V_{1}$ satisfy $(2.12)$ subject to the far-field condition (2.15). Since $x_{0}^{\prime}=-2 \pi \gamma(S) S \nabla R$, we can write this problem for $W_{1}=\left(V_{1}, U_{1}\right)^{t}$ in $y \in \mathbb{R}^{2}$ as

$$
\Delta_{y} W_{1}+\mathcal{M}_{0} W_{1}=\gamma(S)\left(\begin{array}{c}
2 \operatorname{Re}\left(g e^{i \theta}\right) V^{\prime} \\
0
\end{array}\right), \quad W_{1} \sim\left(\begin{array}{c}
0 \\
-2 \operatorname{Re}\left(g e^{i \theta}\right) \rho
\end{array}\right), \quad \text { as } \quad \rho \rightarrow \infty,
$$

where $\mathcal{M}_{0}$ is the matrix defined in $(2.12 b)$. Here Re indicates the real part, and $g$ is the complex constant defined by

$$
g \equiv \pi S\left(R_{x_{1}}-i R_{x_{2}}\right),
$$

where $\nabla R=\left(R_{x_{1}}, R_{x_{2}}\right)$ is the gradient of the regular part of the Neumann Green's function at $x_{0}$. Therefore,

$$
W_{1}=\left(V_{1}, U_{1}\right)^{t}=g e^{i \theta} \hat{W}_{1}(\rho)+\text { c.c }, \quad \hat{W}_{1}(\rho)=\left(\hat{V}_{1}(\rho), \hat{U}_{1}(\rho)\right)^{t},
$$

where c.c denotes complex conjugate. Here $\hat{W}_{1}(\rho)$ is the real-valued radially symmetric vector function satisfying

$$
\hat{W}_{1 \rho \rho}+\frac{1}{\rho} \hat{W}_{1 \rho}-\frac{1}{\rho^{2}} \hat{W}_{1}+\mathcal{M}_{0} \hat{W}_{1}=\gamma(S)\left(\begin{array}{c}
V^{\prime} \\
0
\end{array}\right), \quad 0 \leq \rho<\infty, \quad \hat{W}_{1} \sim\left(\begin{array}{c}
0 \\
-\rho
\end{array}\right), \quad \text { as } \quad \rho \rightarrow \infty .
$$

Next, we derive the eigenvalue problem by susbtituting

$$
u=\frac{1}{\sqrt{D}} \mathcal{U}+\frac{e^{\lambda t}}{D} N(y), \quad v=\sqrt{D} \mathcal{V}+e^{\lambda t} \Phi(y), \quad y=\varepsilon^{-1}\left[x-x_{0}(\tau)\right], \quad \tau=\varepsilon^{2} t,
$$

into (1.2), and then retaining linear terms in $N$ and $\Phi$. Accurate to within terms of order $\mathcal{O}(\varepsilon)$, we obtain that

$$
\Delta_{y} \Phi-\Phi+2 \mathcal{U} \mathcal{V} \Phi+\mathcal{V}^{2} N=\lambda \Phi-\varepsilon \nabla \Phi \cdot x_{0}^{\prime}, \quad \Delta_{y} N-\mathcal{V}^{2} N-2 \mathcal{U} \mathcal{V} \Phi=0 .
$$

Here the gradient term of order $\mathcal{O}(\varepsilon)$ in the equation for $\Phi$ arises as a result of the dependence of $y$ on the spot 
trajectory $x_{0}\left(\varepsilon^{2} t\right)$. For $S \approx \Sigma_{2}$, we then expand

$$
\Phi=\Phi_{0}+\varepsilon \Phi_{1}+\cdots, \quad N=N_{0}+\varepsilon N_{1}+\cdots, \quad \lambda=\varepsilon \lambda_{1}+\cdots .
$$

Upon subsitituting $(2.35)$ and $(2.29 b)$ into $(2.34)$, and collecting powers of $\varepsilon$, we obtain for $y \in \mathbb{R}^{2}$ that

$$
\begin{gathered}
\Delta_{y} P_{0}+\mathcal{M}_{0} P_{0}=0, \quad P_{0} \equiv\left(\Phi_{0}, N_{0}\right)^{t}, \\
\Delta_{y} P_{1}+\mathcal{M}_{0} P_{1}+\mathcal{M}_{1} P_{0}=\Lambda_{1} P_{0}-\mathcal{F} P_{0}, \quad P_{1} \equiv\left(\Phi_{1}, N_{1}\right)^{t} .
\end{gathered}
$$

Here the matrices $\mathcal{M}_{1}, \mathcal{F}$, and $\Lambda_{1}$, are defined by

$$
\mathcal{M}_{1} \equiv\left(\begin{array}{cc}
2 U_{1} V+2 U V_{1} & 2 V V_{1} \\
-2 U_{1} V-2 U V_{1} & -2 V V_{1}
\end{array}\right), \quad \Lambda_{1} \equiv\left(\begin{array}{cc}
\lambda_{1} & 0 \\
0 & 0
\end{array}\right), \quad \mathcal{F} \equiv\left(\begin{array}{cc}
x_{0}^{\prime} \cdot \nabla & 0 \\
0 & 0
\end{array}\right) .
$$

Upon using (2.31) for $W_{1}=\left(V_{1}, U_{1}\right)^{t}$, and upon noting that $x_{0}^{\prime} \cdot \nabla=-2 \gamma(S) \operatorname{Re}\left(g e^{i \theta}\right) \partial_{\rho}$, we can write $\mathcal{M}_{1}$ and $\mathcal{F}$ as

$$
\mathcal{M}_{1}=g e^{i \theta} \hat{\mathcal{M}}_{1}+\text { c.c }, \quad \mathcal{F}=-\gamma(S)\left(g e^{i \theta}+\text { c.c }\right) \hat{\mathcal{F}}, \quad \hat{\mathcal{F}} \equiv\left(\begin{array}{cc}
\partial_{\rho} & 0 \\
0 & 0
\end{array}\right) .
$$

Here $\hat{\mathcal{M}}_{1}$ is the real-valued $2 \times 2$ matrix, with radially symmetric matrix entries, obtained by replacing $V_{1}$ and $U_{1}$ in (2.37) with $\hat{V}_{1}$ and $\hat{U}_{1}$, respectively.

When $S=\Sigma_{2}$ the leading-order problem (2.36a) admits four independent nontrivial solutions. We write these solutions in complex form as

$$
P_{0}=A \hat{P}_{01}(\rho) e^{i \theta}+B \hat{P}_{02}(\rho) e^{2 i \theta}+\text { c.c. }, \quad \hat{P}_{0 j}(\rho)=\left(\hat{\Phi}_{0 j}(\rho), \hat{N}_{0 j}(\rho)\right)^{t}, \quad j=1,2 .
$$

Here $A$ and $B$ are complex constants to be determined, $\rho=|y|, \tan (\theta)=y_{2} / y_{1}$, and c.c. denotes the complex conjugate. In addition, $\hat{P}_{0 j}(\rho)$ for $j=1,2$ are the real-valued radially symmetric functions satisfying

$$
\left(\hat{P}_{0 j}\right)_{\rho \rho}+\frac{1}{\rho}\left(\hat{P}_{0 j}\right)_{\rho}-\frac{j^{2}}{\rho^{2}} \hat{P}_{0 j}+\mathcal{M}_{0} \hat{P}_{0 j}=0, \quad 0 \leq \rho<\infty ; \quad \hat{P}_{0 j} \rightarrow 0 \quad \text { as } \quad \rho \rightarrow \infty .
$$

As a result of translation invariance, it readily follows that $\hat{P}_{01}$ is given explicitly by

$$
\hat{P}_{01}=\left(\hat{\Phi}_{01}, \hat{N}_{01}\right)^{t}=\left(V^{\prime}, U^{\prime}\right)^{t}
$$

where $V$ and $U$ satisfy the core problem $(2.3 a)$ when $S=\Sigma_{2}$. The solution $\hat{W}_{1}$ to $(2.30 a)$ has the form $\hat{W}_{1}=$ $\hat{W}_{1 p}+\beta \hat{P}_{01}$, where $\hat{W}_{1 p}$ is the particular solution to $(2.30 a)$, and $\beta$ is any constant. Without loss of generality, we impose that $\beta=0$ so that $\hat{W}_{1}$ is uniquely determined.

Since $(2.36 a)$ has four independent solutions, the corresponding adjoint problem for $y \in \mathbb{R}^{2}$ given by

$$
\Delta_{y} P^{*}+\mathcal{M}_{0}^{t} P^{*}=0, \quad P^{*} \rightarrow 0 \quad \text { as }|y| \rightarrow \infty, \quad P^{*} \equiv\left(\Phi^{*}, \Psi^{*}\right)^{t},
$$

admits a four-dimensional null space. We write these four independent solutions in complex form as

$$
P_{11}^{*} \equiv \hat{P}_{1}^{*}(\rho) e^{i \theta}+\text { c.c }, \quad P_{12}^{*} \equiv \hat{P}_{1}^{*}(\rho) i e^{i \theta}+\text { c.c }, \quad P_{21}^{*} \equiv \hat{P}_{2}^{*}(\rho) e^{2 i \theta}+\text { c.c }, \quad P_{22}^{*} \equiv \hat{P}_{2}^{*}(\rho) i e^{2 i \theta}+\text { c.c } .
$$

Here $\hat{P}_{j}^{*}(\rho)=\left(\hat{\Phi}_{j}^{*}(\rho), \hat{N}_{j}^{*}(\rho)\right)^{t}$ for $j=1,2$ are real-valued radially symmetric functions satisfying

$$
\left(\hat{P}_{j}^{*}\right)_{\rho \rho}+\frac{1}{\rho}\left(\hat{P}_{j}^{*}\right)_{\rho}-\frac{j^{2}}{\rho^{2}} \hat{P}_{j}^{*}+\mathcal{M}_{0}^{t} \hat{P}_{j}^{*}=0, \quad 0 \leq \rho<\infty ; \quad \hat{P}_{j}^{*} \rightarrow 0 \quad \text { as } \quad \rho \rightarrow \infty .
$$

Next, we impose four solvability conditions for the solution $P_{1}$ to $(2.36 b)$. Upon multiplying $(2.36 b)$ by $P_{j k}^{*}$, and then integrating the resulting expression by parts over $\mathbb{R}^{2}$, we obtain that

$$
J_{j k}=I_{j k}+F_{j k}, \quad j, k=1,2,
$$


where the integrals $J_{j k}, I_{j k}$, and $F_{j k}$, are defined explicitly by

$$
J_{j k} \equiv \int_{\mathbb{R}^{2}}\left(P_{j k}^{*}\right)^{t} \Lambda_{1} P_{0} d y, \quad I_{j k} \equiv \int_{\mathbb{R}^{2}}\left(P_{j k}^{*}\right)^{t} \mathcal{M}_{1} P_{0} d y, \quad F_{j k} \equiv \int_{\mathbb{R}^{2}}\left(P_{j k}^{*}\right)^{t} \mathcal{F} P_{0} d y .
$$

We then substitute (2.43), (2.39), and (2.38), into (2.45 b), and calculate the resulting integrals. The only integrals that do not vanish are the ones for which the integrand is radially symmetric. In this way, we obtain that

$$
\begin{gathered}
I_{11}=4 \pi \operatorname{Re}(g \bar{B}) \int_{0}^{\infty} \rho\left(\hat{P}_{1}^{*}\right)^{t} \hat{\mathcal{M}}_{1} \hat{P}_{02} d \rho, \quad I_{12}=4 \pi \operatorname{Re}(i g \bar{B}) \int_{0}^{\infty} \rho\left(\hat{P}_{1}^{*}\right)^{t} \hat{\mathcal{M}}_{1} \hat{P}_{02} d \rho, \\
I_{21}=4 \pi \operatorname{Re}(g A) \int_{0}^{\infty} \rho\left(\hat{P}_{2}^{*}\right)^{t} \hat{\mathcal{M}}_{1} \hat{P}_{01} d \rho, \quad I_{22}=4 \pi \operatorname{Re}(-i g A) \int_{0}^{\infty} \rho\left(\hat{P}_{2}^{*}\right)^{t} \hat{\mathcal{M}}_{1} \hat{P}_{01} d \rho .
\end{gathered}
$$

Here the overbar on $B$ denotes complex conjugate. In a similar way, we calculate

$$
\begin{gathered}
J_{11}=4 \pi \operatorname{Re}(A) \lambda_{1} \int_{0}^{\infty} \rho \hat{\Phi}_{1}^{*} \hat{\Phi}_{01} d \rho, \quad J_{12}=4 \pi \operatorname{Re}(i \bar{A}) \lambda_{1} \int_{0}^{\infty} \rho \hat{\Phi}_{1}^{*} \hat{\Phi}_{01} d \rho, \\
J_{21}=4 \pi \operatorname{Re}(B) \lambda_{1} \int_{0}^{\infty} \rho \hat{\Phi}_{2}^{*} \hat{\Phi}_{02} d \rho, \quad J_{22}=4 \pi \operatorname{Re}(i \bar{B}) \lambda_{1} \int_{0}^{\infty} \rho \hat{\Phi}_{2}^{*} \hat{\Phi}_{02} d \rho
\end{gathered}
$$

and

$$
\begin{aligned}
& F_{11}=-4 \pi \gamma(S) \operatorname{Re}(g \bar{B}) \int_{0}^{\infty} \rho\left(\hat{P}_{1}^{*}\right)^{t} \hat{\mathcal{F}} \hat{P}_{02} d \rho, \quad F_{12}=-4 \pi \gamma(S) \operatorname{Re}(i g \bar{B}) \int_{0}^{\infty} \rho\left(\hat{P}_{1}^{*}\right)^{t} \hat{\mathcal{F}} \hat{P}_{02} d \rho, \\
& F_{21}=-4 \pi \gamma(S) \operatorname{Re}(g A) \int_{0}^{\infty} \rho\left(\hat{P}_{2}^{*}\right)^{t} \hat{\mathcal{F}} \hat{P}_{01} d \rho, \quad F_{22}=-4 \pi \gamma(S) \operatorname{Re}(-i g A) \int_{0}^{\infty} \rho\left(\hat{P}_{2}^{*}\right)^{t} \hat{\mathcal{F}} \hat{P}_{01} d \rho
\end{aligned}
$$

Finally, upon substituting (2.46), (2.47), and (2.48), into the solvability conditions $(2.45 a)$, we obtain

$$
\lambda_{1} \operatorname{Re}(A) \kappa_{1}=\operatorname{Re}(g \bar{B}), \quad \lambda_{1} \operatorname{Re}(i \bar{A}) \kappa_{1}=\operatorname{Re}(i g \bar{B}), \quad \lambda_{1} \operatorname{Re}(B) \kappa_{2}=\operatorname{Re}(g A), \quad \lambda_{1} \operatorname{Re}(i \bar{B}) \kappa_{2}=\operatorname{Re}(-i g A) .
$$

Here $\kappa_{1}$ and $\kappa_{2}$ are the real constants defined by

$$
\kappa_{1} \equiv \frac{\int_{0}^{\infty} \rho \hat{\Phi}_{1}^{*} \hat{\Phi}_{01} d \rho}{\int_{0}^{\infty} \rho\left(\hat{P}_{1}^{*}\right)^{t}\left[\hat{\mathcal{M}}_{1}-\gamma(S) \hat{\mathcal{F}}\right] \hat{P}_{02} d \rho}, \quad \kappa_{2} \equiv \frac{\int_{0}^{\infty} \rho \hat{\Phi}_{2}^{*} \hat{\Phi}_{02} d \rho}{\int_{0}^{\infty} \rho\left(\hat{P}_{2}^{*}\right)^{t}\left[\hat{\mathcal{M}}_{1}-\gamma(S) \hat{\mathcal{F}}\right] \hat{P}_{01} d \rho} .
$$

Without loss of generality, we can assume that the complex constant $g$, defined in $(2.30 \mathrm{~b})$, is real and positive so that the motion of the spot is directed along the $y_{1}$ axis. More generally, this can always be achieved by multiplying the original coordinate vector $\left(y_{1}, y_{2}\right)^{t}$ by an appropriate orthogonal matrix. Assuming that $g$ is real and positive, we then write $A=A_{r}+i A_{i}$ and $B=B_{r}+i B_{i}$ to obtain that (2.49) reduces to

$$
\lambda_{1} A_{r} \kappa_{1}=g B_{r}, \quad \lambda_{1} A_{i} \kappa_{1}=g B_{i}, \quad \lambda_{1} B_{r} \kappa_{2}=g A_{r}, \quad \lambda_{1} B_{i} \kappa_{2}=g A_{i} .
$$

In this way, (2.49) decomposes into two $2 \times 2$ matrix eigenvalue problems. The first such problem is

$$
\left(\begin{array}{cc}
0 & g / \kappa_{1} \\
g / \kappa_{2} & 0
\end{array}\right)\left(\begin{array}{c}
A_{r} \\
B_{r}
\end{array}\right)=\lambda_{1}\left(\begin{array}{c}
A_{r} \\
B_{r}
\end{array}\right)
$$

while the second eigenvalue problem is obtain by replacing $A_{r}$ and $B_{r}$ with $A_{i}$ and $B_{i}$, respectively. The eigenvalues of this matrix problem are

$$
\lambda_{1}= \pm \frac{g}{\sqrt{\kappa_{1} \kappa_{2}}} .
$$

Our numerical computations described below show that $\kappa_{1}<0$ and $\kappa_{2}<0$. Therefore, the unstable eigenvalue is 
$\lambda_{1}=+g / \sqrt{\kappa_{1} \kappa_{2}}$ and the corresponding eigenvector satisfies

$$
B=\kappa A, \quad \kappa \equiv \frac{\kappa_{1}}{\sqrt{\kappa_{1} \kappa_{2}}} .
$$

Hence, the first component of $P_{0}$ in (2.39) is proportional to

$$
\Phi_{0}=\hat{\Phi}_{01}(\rho) \cos (\theta)+\kappa \hat{\Phi}_{02}(\rho) \cos (2 \theta) .
$$

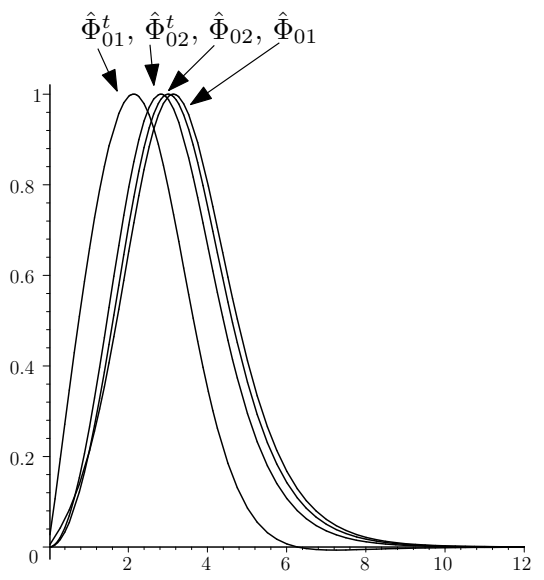

(a)

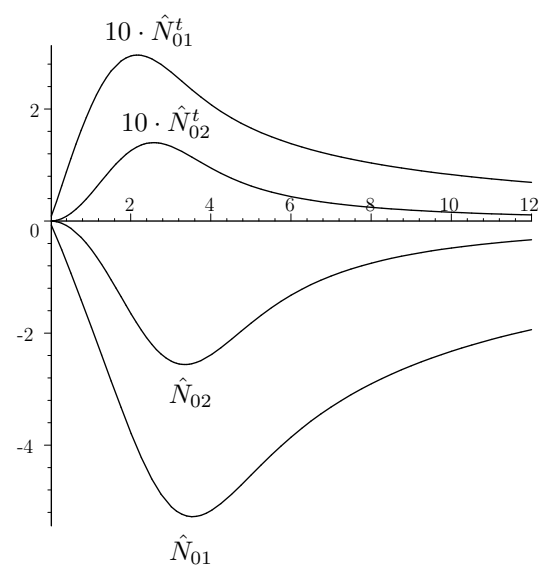

(b)

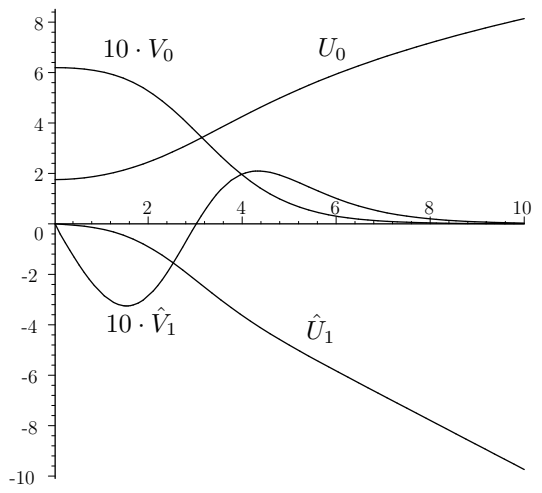

(c)

FiguRE 5. For $S=\Sigma_{2}=4.3$ we show plots of (a) $\hat{\Phi}_{01}^{*}, \hat{\Phi}_{02}^{*}, \hat{\Phi}_{01}, \hat{\Phi}_{02}$; (b) $\hat{N}_{01}^{*}, \hat{N}_{02}^{*}, \hat{N}_{02}, \hat{N}_{01}$; and (c) $U, \hat{U}_{1}, V, \hat{V}_{1}$.

In Fig. 5 we plot $\hat{\Phi}_{01}(\rho)$ and $\hat{\Phi}_{02}(\rho)$ obtained by solving (2.40) numerically. Since we scaled them to have a maximum of one, it follows that the direction of the splitting (controlled by $\cos 2 \theta$ ) is perpendicular to the direction of the motion (controlled by $\cos \theta$ ) provided that $\kappa$ in $(2.55)$ is negative.

Finally, we outline our numerical approach for showing that $\kappa_{j}$, for $j=1,2$, as defined in (2.50), are both negative when $S=\Sigma_{2} \approx 4.3$, and hence $\kappa<0$ in (2.55). For this value of $S$, we compute numerically from (2.16) that $\gamma(S) \approx 1.703$. First, we compute $U, V$ and $\hat{V}_{1}, \hat{U}_{1}$ using Maple's boundary value problem solver. Next, we compute the eigenfunctions corresponding to $m=1$ and $m=2$ and their adjoints from (2.40) and (2.44), respectively. All of these problems are ODE problems. To solve them numerically, we discretized the radial Laplacian using central differences on a grid of $N$ points and solved the corresponding $2 N$-dimensional matrix eigenvalue problem. The resulting matrix is 5-banded, and its spectrum is easily computed using Maple's linear algebra package. We performed the computations with $N=200$ on an interval [0,12]. Doubling $N$ or the interval length did not affect the answer in the first three significant digits. The graphs of $U, V, \hat{V}_{1}$, and $\hat{U}_{1}$, are shown in Fig. $5(\mathrm{c})$. The graphs of eigenfunctions and their adjoints are shown in Fig. 5(a,b). This numerical procedure yields the results

$$
\kappa_{1}=-0.926, \quad \kappa_{2}=-1.800, \quad \kappa=-0.717,
$$

so that $\kappa$ is indeed negative. The constant $\kappa$ measures the rate at which the spot splits. Since $\kappa$ is independent of $g$, the rate of splitting is proportional to the velocity, with $\kappa$ being the constant of proportionality.

We now compare our theoretical prediction for the direction of spot-splitting with that obtained from a full numerical simulation of (1.2) in the unit disk for the parameter values $\varepsilon=0.03, D=1$, and $a=8.8$. The initial spot location is taken to be at $(0.5,0)$. Since $|\Omega|=\pi$, we calculate from $(2.6)$ that $S=4.4>\Sigma_{2} \approx 4.3$. Our prediction of 
spot-splitting is confirmed in Fig. 6, where we plot the the position of the spot at increments of 5 time units. Spot self-replication is observed at $t \approx 100$. Notice that the two newly created spots move in a direction orthogonal to the motion of the original spot.

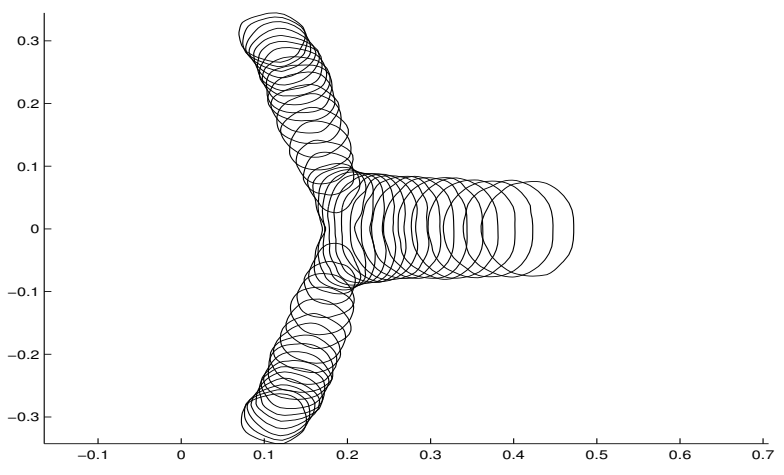

(a) contour plot of $v$

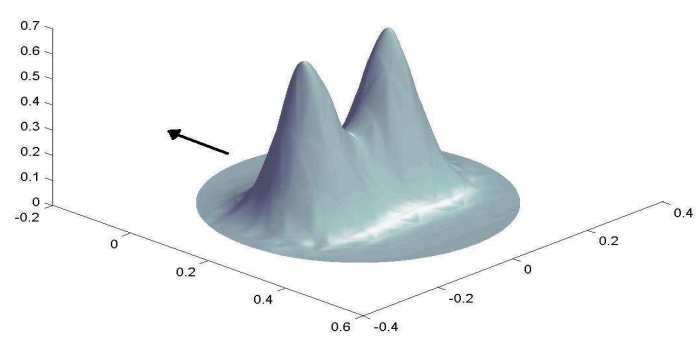

(b) spatial profile of $v$

Figure 6. Spot-splitting for (1.2) in the unit disk for the parameter values $\varepsilon=0.03, D=1$, and $a=8.8$. (a) Trace of the contour $v=0.5$ from $t=15$ to $t=175$ with increments $\Delta t=5$. Spot-splitting is perpendicular to the direction of motion. (b) The spatial profile of the spot at $t=105$ during the splitting event.

\section{Multi-Spot Solutions}

We now extend the analysis in $\S 2$ in order to construct a quasi-equilibrium solution to (1.2) with $K$ spots and to derive an ODE system governing its slow evolution. To construct a $K$-spot quasi-equilibrium solution we "freeze" the spots at locations $x_{1}, \ldots, x_{K}$ with $x_{j} \in \Omega$ for $j=1, \ldots, K$. We also assume that the distance between any two spots is $\mathcal{O}(1)$ as $\varepsilon \rightarrow 0$. In the inner region near the $j^{\text {th }}$ spot we introduce the new variables

$$
u=\frac{1}{\sqrt{D}} \mathcal{U}_{j}, \quad v=\sqrt{D} \mathcal{V}_{j}, \quad y=\varepsilon^{-1}\left(x-x_{j}\right) .
$$

As in $\S 2.1$, to leading order we look for a radially symmetric solution of the form $\mathcal{U}_{j} \sim U_{j}(\rho)$ and $\mathcal{V}_{j} \sim V_{j}(\rho)$ with $\rho=|y|$. Thus, for each $j=1, \ldots, K$, we have that $U_{j}$ and $V_{j}$, with primes denoting derivatives in $\rho$, satisfy

$$
\begin{gathered}
V_{j}^{\prime \prime}+\frac{1}{\rho} V_{j}^{\prime}-V_{j}+U_{j} V_{j}^{2}=0, \quad U_{j}^{\prime \prime}+\frac{1}{\rho} U_{j}^{\prime}-U_{j} V_{j}^{2}=0, \quad 0<\rho<\infty, \\
U_{j}^{\prime}(0)=V_{j}^{\prime}(0)=0 ; \quad V_{j} \rightarrow 0, \quad U_{j} \sim S_{j} \log \rho+\chi\left(S_{j}\right) \quad \text { as } \quad \rho \rightarrow \infty .
\end{gathered}
$$

The function $\chi(S)$ was computed numerically in $\S 2.1$ (see Fig. 2(b))).

The source strengths $S_{j}$, for $j=1, \ldots, K$, are determined by matching the solution to the core problems (3.2) to an outer solution for $u$. By proceeding as in $\S 2.1$ (see equation (2.4)), we can readily derive the outer problem

$$
\begin{aligned}
\Delta u & =-\frac{a}{D}+\frac{2 \pi}{\sqrt{D}} \sum_{j=1}^{K} S_{j} \delta\left(x-x_{j}\right), \quad x \in \Omega ; \quad \partial_{n} u=0, \quad x \in \partial \Omega, \\
u & \sim \frac{1}{\sqrt{D}}\left[S_{j} \log \left|x-x_{j}\right|+\chi\left(S_{j}\right)+\frac{S_{j}}{\nu}\right] \quad \text { as } \quad x \rightarrow x_{j}, \quad j=1, \ldots, K,
\end{aligned}
$$


where $\nu \equiv-1 / \log \varepsilon$. The Divergence theorem enforces that $2 \pi \sum_{j=1}^{K} S_{j}=a|\Omega| / \sqrt{D}$, and the solution to (3.3) is

$$
u(x)=-\frac{2 \pi}{\sqrt{D}}\left(\sum_{i=1}^{K} S_{i} G\left(x ; x_{i}\right)+u_{c}\right) .
$$

Here $u_{c}$ is a constant to be found and $G\left(x ; x_{i}\right)$ is the Neumann Green's function satisfying (2.7). As in $\S 2$, we use $(2.7 b)$ to get the near-field behavior of the outer solution as $x \rightarrow x_{j}$. Upon matching this near-field behavior of the outer solution with the far-field behavior of each leading order core solution $U_{j}$, we obtain for each $j=1, \ldots, K$ that

$$
S_{j} \log \left|x-x_{j}\right|-2 \pi S_{j} R\left(x_{j} ; x_{j}\right)-2 \pi u_{c}-2 \pi \sum_{\substack{i=1 \\ i \neq j}}^{K} S_{i} G\left(x_{j} ; x_{i}\right) \sim S_{j} \log \left|x-x_{j}\right|+\chi\left(S_{j}\right)+\frac{S_{j}}{\nu} .
$$

These matching conditions gives $K$ equations relating $S_{j}$ and $u_{c}$. We summarize our construction as follows:

Principal Result 3.1: For given spot locations $x_{j}$ for $j=1, \ldots, K$, let $S_{j}$ for $j=1, \ldots, K$ and $u_{c}$ satisfy the nonlinear algebraic system

$$
S_{j}+2 \pi \nu\left(S_{j} R_{j, j}+\sum_{\substack{i=1 \\ i \neq j}}^{K} S_{i} G_{j, i}\right)+\nu \chi\left(S_{j}\right)=-2 \pi \nu u_{c} ; \quad \sum_{j=1}^{K} S_{j}=\frac{a|\Omega|}{2 \pi \sqrt{D}} .
$$

Here $\nu \equiv-1 / \log \varepsilon$ with $G_{j, i} \equiv G\left(x_{j} ; x_{i}\right)$ and $R_{j, j} \equiv R\left(x_{j} ; x_{j}\right)$, where $G$ is the Neumann Green's function of (2.7) with regular part $R$. The nonlinear term $\chi\left(S_{j}\right)$ in (3.6) is as given in (3.2b) (see Fig. 2(b)). Then, for $\varepsilon \rightarrow 0$, the outer solution for a $K$-spot quasi-equilibrium solution is given by (3.4) and the leading order inner solutions are given by $u \sim D^{-1 / 2} U_{j}$ and $v \sim \sqrt{D} V_{j}$, where $U_{j}$ and $V_{j}$ is the solution to core problem (3.2).

We emphasize that the system (3.6) contains all of the logarithmic correction terms of order $\mathcal{O}\left(\nu^{k}\right)$ for any $k$ that are required in the construction of the quasi-equilibrium solution. Hence, we say that (3.6) has "summed" all of the logarithmic terms in powers of $\nu$ for the source strengths $S_{j}, j=1, \ldots, K$. Related, but linear, algebraic systems of equations determining unknown source strengths arising from a singular perturbation analysis of certain linear steady-state diffusion problems on perforated two-dimensional domains have been derived in $[\mathbf{8}],[\mathbf{2 6}]$, and $[\mathbf{4 2}]$.

It is convenient to write (3.6) in matrix form as

$$
(I+2 \pi \nu \mathcal{G}) S_{v}+\nu \chi_{v}=-2 \pi \nu u_{c} e ; \quad e^{t} S_{v}=\frac{a|\Omega|}{2 \pi \sqrt{D}} .
$$

Here $I$ is the identity matrix, $\mathcal{G}$ is the Green's matrix, and the vectors $S_{v}, \chi_{v}$, and $e$, are defined by

$$
\mathcal{G} \equiv\left(\begin{array}{cccc}
R_{1,1} & G_{1,2} & \cdots & G_{1, K} \\
G_{2,1} & \ddots & \ddots & \vdots \\
\vdots & \ddots & \ddots & G_{K-1, K} \\
G_{K, 1} & \cdots & G_{K, K-1} & R_{K, K}
\end{array}\right), \quad S_{v} \equiv\left(\begin{array}{c}
S_{1} \\
\vdots \\
S_{K}
\end{array}\right), \quad e \equiv\left(\begin{array}{c}
1 \\
\vdots \\
1
\end{array}\right), \quad \chi_{v} \equiv\left(\begin{array}{c}
\chi\left(S_{1}\right) \\
\vdots \\
\chi\left(S_{K}\right)
\end{array}\right)
$$

By multiplying the first equation in (3.7) by $e^{t}$, and then using the expression for $e^{t} S_{v}$ in (3.7), we can obtain $u_{c}$ as

$$
u_{c}=-\frac{1}{2 \pi K \nu}\left[\frac{a|\Omega|}{2 \pi \sqrt{D}}+2 \pi \nu e^{t} \mathcal{G} S_{v}+\nu e^{t} \chi_{v}\right]
$$

By using this expression in the first equation in (3.7), we can eliminate $u_{c}$ to get an equation solely for $S_{v}$.

Principal Result 3.2: The nonlinear algebraic system in (3.6) can be decoupled into an equation for $S_{v}$ given by

$$
S_{v}+\nu(I-\mathcal{E})\left(\chi_{v}+2 \pi \mathcal{G} S_{v}\right)=\frac{a|\Omega|}{2 \pi K \sqrt{D}} e, \quad \mathcal{E} \equiv \frac{1}{K} e e^{t} .
$$


In terms of $S_{v}$, the constant $u_{c}$ in (3.4) is given in (3.9).

The following condition on the Green's matrix $\mathcal{G}$, which reflects both the symmetry of $\Omega$ and of the configuration of the spot locations $x_{1}, \ldots, x_{K}$, gives a necessary condition for the $K$ spots to have a common source strength $S_{c}$ :

Principal Result 3.3: Suppose that $e=(1, \ldots, 1)^{t}$ is an eigenvector of $\mathcal{G}$, so that

$$
\mathcal{G} e=\frac{p}{K} e, \quad p=p\left(x_{1}, \ldots, x_{K}\right) \equiv \sum_{i=1}^{K} \sum_{j=1}^{K} \mathcal{G}_{i j} .
$$

Then, $S_{v}=S_{c}$ e, where the common (scalar) spot source strength $S_{c}$ and the constant $u_{c}$ are given explicitly by

$$
S_{c} \equiv \frac{a|\Omega|}{2 \pi K \sqrt{D}}, \quad u_{c}=-\frac{a|\Omega|}{4 \pi^{2} K \nu \sqrt{D}}-\frac{S_{c} p}{K}-\frac{\chi\left(S_{c}\right)}{2 \pi} .
$$

Finally, for $\nu \equiv-1 / \log \varepsilon \ll 1$, and for arbitrary spot locations $x_{1}, \ldots, x_{K}$, we can readily derive the following two-term expansion for $S_{v}$ and $u_{c}$ from (3.10) and (3.9) in terms of $S_{c}, \mathcal{G}$ and $p$ :

$$
S_{v} \sim S_{c} e-2 \pi \nu S_{c}\left(\mathcal{G}-\frac{p}{K} I\right) e+\mathcal{O}\left(\nu^{2}\right) ; \quad u_{c} \sim-\frac{a|\Omega|}{4 \pi^{2} K \nu \sqrt{D}}-\frac{S_{c} p}{K}-\frac{\chi\left(S_{c}\right)}{2 \pi}+\mathcal{O}(\nu) .
$$

Next, we proceed as in $\S 2.2$ in order to derive an ODE system for the slow evolution of the spots $x_{j}$ for $j=1, \ldots, K$. Since the analysis is similar to that in $\S 2$ we only give a brief outline of it here. In the inner region near $x=x_{j}$ we expand the solution to $(1.2)$ as

$$
u=\frac{1}{\sqrt{D}}\left(U_{j}(\rho)+\varepsilon U_{1 j}\left(y_{j}\right)+\cdots\right), \quad v=\sqrt{D}\left(V_{j}(\rho)+\varepsilon V_{1 j}\left(y_{j}\right)+\cdots\right), \quad y_{j}=\varepsilon^{-1}\left[x-x_{j}(\tau)\right], \quad \tau=\varepsilon^{2} t .
$$

Here $U_{j}(\rho)$ and $V_{j}(\rho)$, with $\rho=\left|y_{j}\right|$, are the radial symmetric solutions of the core problem (3.2). We then substitute (3.14) into (1.2) and collect terms of order $\mathcal{O}(\varepsilon)$ to derive that $V_{1 j}$ and $U_{1 j}$ for each $j=1, \ldots, K$ satisfies

$$
\Delta_{y_{j}} W_{1 j}+\mathcal{M}_{j} W_{1 j}=f_{j}, \quad y_{j} \in \mathbb{R}^{2},
$$

where $y_{j}=\rho e_{\theta}$, and the vectors $W_{1 j}, f_{j}, e_{\theta}$ and the $2 \times 2$ matrices $\mathcal{M}_{j}$ are defined by

$$
W_{1 j} \equiv\left(\begin{array}{c}
V_{1 j} \\
U_{1 j}
\end{array}\right), \quad f \equiv\left(\begin{array}{c}
-V_{j}^{\prime} x_{j}^{\prime} \cdot e_{\theta} \\
0
\end{array}\right), \quad e_{\theta} \equiv\left(\begin{array}{c}
\cos \theta \\
\sin \theta
\end{array}\right), \quad \mathcal{M}_{j} \equiv\left(\begin{array}{cc}
-1+2 U_{j} V_{j} & V_{j}^{2} \\
-2 U_{j} V_{j} & -V_{j}^{2}
\end{array}\right) .
$$

The determination of a far-field condition for $W_{1 j}$ is derived as in $\S 2.2$ by performing a higher order matching of the outer and inner solutions. In this way, we obtain that the solution to (3.15) must satisfy

$$
W_{1 j} \sim\left(\begin{array}{c}
0 \\
\alpha_{j} \cdot y_{j}
\end{array}\right) \quad \text { as } \quad y_{j} \rightarrow \infty, \quad \alpha_{j} \equiv-2 \pi S_{j} \nabla R\left(x_{j} ; x_{j}\right)-2 \pi \sum_{\substack{i=1 \\
i \neq j}}^{K} S_{i} \nabla G\left(x_{j} ; x_{i}\right) .
$$

As shown in Principal Result 2.1, the problem (3.15) subject to (3.16) determines $x_{j}^{\prime}$ in terms of the vector $\alpha_{j}$. In this way, we obtain the following main result for the dynamics of a $K$-spot quasi-equilibrium solution.

Principal Result 3.4: For $\varepsilon \rightarrow 0$ the slow dynamics of a collection $x_{1}, \ldots, x_{K}$ of spots satisfies the differentialalgebraic system $(D A E)$,

$$
x_{j}^{\prime} \sim-2 \pi \varepsilon^{2} \gamma\left(S_{j}\right)\left(S_{j} \nabla R\left(x_{j} ; x_{j}\right)+\sum_{\substack{i=1 \\ i \neq j}}^{K} S_{i} \nabla G\left(x_{j} ; x_{i}\right)\right), \quad j=1, \ldots, K .
$$

Here the source strengths $S_{j}$, for $j=1, \ldots, K$, are determined in terms of $x_{1}, \ldots, x_{K}$ by the nonlinear algebraic system (3.6). The function $\gamma\left(S_{j}\right)$, plotted in Fig. 3(a), is defined in (2.16) of Principal Result 2.1. 


\subsection{The Stability of a $K$-Spot Quasi-Equilibrium Solution}

To determine the stability of the quasi-equilibrium $K$-spot solution to fast $\mathcal{O}(1)$ time-scale instabilities we proceed as in $\S 2.3$. We let $u_{e}, v_{e}$ be the quasi-equilibrium solution, and we introduce the perturbation (2.22) to derive the eigenvalue problem (2.23). Following $§ 2.3$, in the inner region near the $j^{\text {th }}$ spot we introduce $N_{j}$ and $\Phi_{j}$ by

$$
\eta=e^{i m \theta} N_{j}(\rho), \quad \phi=e^{i m \theta} \Phi_{j}(\rho), \quad \rho=|y|, \quad y=\varepsilon^{-1}\left(x-x_{j}\right),
$$

where $y^{t}=\rho(\cos \theta, \sin \theta)$. Substituting (3.18) into (2.23), and by using $u_{e} \sim D^{-1 / 2} U_{j}(\rho)$ and $v_{e} \sim \sqrt{D} V_{j}(\rho)$, where $U_{j}$ and $V_{j}$ satisfy the core problem (3.2), we obtain in terms of the operator $\mathcal{L}_{m} \Phi \equiv \partial_{\rho \rho} \Phi+\rho^{-1} \partial_{\rho} \Phi-m^{2} \rho^{-2} \Phi$ that

$$
\mathcal{L}_{m} \Phi_{j}-\Phi_{j}+2 U_{j} V_{j} \Phi_{j}+D V_{j}^{2} N_{j}=\lambda \Phi_{j}, \quad \mathcal{L}_{m} N_{j}-V_{j}^{2} N_{j}=\frac{2}{D} U_{j} V_{j} \Phi_{j}, \quad 0 \leq \rho<\infty .
$$

First consider the modes with $m \geq 2$. For these modes, we can impose that $\Phi_{j}$ decays exponentially as $\rho \rightarrow \infty$ and that $N_{j} \sim \mathcal{O}\left(\rho^{-m}\right) \rightarrow 0$ as $\rho \rightarrow \infty$. Therefore, for $m \geq 2$ there is no inter-spot coupling through the outer solution for $\eta$, and hence the stability problem reduces to the one studied numerically in $\S 2.3$ for a one-spot solution. The numerical results of $\S 2.3$ and Table 1 then show that the smallest value of $S_{j}$ for which an instability occurs is at the threshold value $S_{j}=\Sigma_{2}$, which corresponds to a peanut-splitting instability.

Next, we derive an eigenvalue problem for the $m=0$ mode that has the effect of coupling the local spot problems (3.19) for $j=1, \ldots, K$ through the outer solution for $\eta$. When $m=0$, (3.19) becomes

$$
\Phi_{j}^{\prime \prime}+\frac{1}{\rho} \Phi_{j}^{\prime}-\Phi_{j}+2 U_{j} V_{j} \Phi_{j}+D V_{j}^{2} N_{j}=\lambda \Phi_{j}, \quad N_{j}^{\prime \prime}+\frac{1}{\rho} N_{j}^{\prime}-V_{j}^{2} N_{j}=\frac{2}{D} U_{j} V_{j} \Phi_{j}, \quad 0 \leq \rho<\infty .
$$

Although $\Phi_{j} \rightarrow 0$ exponentially as $\rho \rightarrow \infty$, we must allow as in $\S 2.3$ that $N_{j} \sim C_{j} \log \rho$ as $\rho \rightarrow \infty$ for some constant $C_{j}$. In terms of this solution, we compute a $B_{j}$ such that

$$
N_{j} \sim C_{j} \log \rho+B_{j} \quad \text { as } \quad \rho \rightarrow \infty .
$$

Since (3.20) is a linear homogeneous problem, it follows that

$$
B_{j}=\hat{B}_{j} C_{j}
$$

where $\hat{B}_{j} \equiv \hat{B}_{j}\left(\lambda, D, S_{j}\right)$. By using the Divergence theorem on the $N_{j}$ equation in (3.20), we identify $C_{j}$ as

$$
C_{j}=\int_{0}^{\infty}\left(V_{j}^{2} N_{j}+\frac{2}{D} U_{j} V_{j} \Phi_{j}\right) \rho d \rho .
$$

By proceeding as in (2.27) of $\S 2.3$, we derive in place of (2.28) that the outer eigenfunction component $\eta$ satisfies

$$
\Delta \eta=2 \pi \sum_{j=1}^{K} C_{j} \delta\left(x-x_{j}\right), \quad x \in \Omega ; \quad \partial_{n} \eta=0, \quad x \in \partial \Omega .
$$

Therefore, $\sum_{j=1}^{K} C_{j}=0$ by the Divergence theorem. We then write the solution to (3.23) as

$$
\eta=-2 \pi \sum_{j=1}^{K} C_{j} G\left(x ; x_{j}\right)+\nu^{-1} \bar{\eta} .
$$

Here $\bar{\eta}$ is an unknown constant and $G(x ; \xi)$ is the Neumann Green's function of (2.7). By expanding the outer solution $\eta$ as $x \rightarrow x_{j}$ and then equating it with the far-field form of the inner solution $N_{j}$ as $|y|=\varepsilon^{-1}\left|x-x_{j}\right| \rightarrow \infty$ given in 
$(3.20 b)$, we obtain the following matching condition for each $j=1, \ldots, K$ :

$$
C_{j} \log \left|x-x_{j}\right|-2 \pi C_{j} R_{j, j}-2 \pi \sum_{\substack{i=1 \\ i \neq j}}^{K} C_{i} G_{j, i}+\frac{\bar{\eta}}{\nu} \sim C_{j} \log \left|x-x_{j}\right|+\frac{C_{j}}{\nu}+B_{j} .
$$

Here $G_{j, i} \equiv G\left(x_{j} ; x_{i}\right)$, and $R_{j, j}=R\left(x_{j} ; x_{j}\right)$, where $R$ is the regular part of $G$. In this way, we obtain the following system for $C_{j}, j=1, \ldots, K$, and $\bar{\eta}$ :

$$
C_{j}+\nu B_{j}+2 \pi \nu C_{j} R_{j, j}+2 \pi \nu \sum_{\substack{i=1 \\ i \neq j}}^{K} C_{i} G_{j, i}=\bar{\eta}, \quad j=1, \ldots, K ; \quad \sum_{j=1}^{K} C_{j}=0 .
$$

Finally, we use (3.21) for $B_{j}$ to write (3.26) as a homogeneous linear system for $c \equiv\left(C_{1}, \ldots, C_{K}\right)^{t}$. By taking the inner product with $e=(1, \ldots, 1)^{t}$, and then using the constraint $e^{t} c=0$, we can isolate $\bar{\eta}$ as

$$
\bar{\eta}=\left(\frac{1}{e^{t} \mathcal{B}^{-1} e}\right) e^{t} \mathcal{B}^{-1}(I+2 \pi \nu \mathcal{G}) c .
$$

Here $\mathcal{G}$ is the Green's matrix of (3.8), and $\mathcal{B}$ is the diagonal matrix with diagonal entries $\mathcal{B}_{j j}=\hat{B}_{j}$ for $j=1, \ldots, K$. By using (3.27) to eliminate $\bar{\eta}$ in (3.26), we readily obtain the following homogeneous linear system for $c$ :

$$
\mathcal{A} c=0, \quad \mathcal{A} \equiv\left(I-\frac{K}{e^{t} \mathcal{B}^{-1} e} \mathcal{E} \mathcal{B}^{-1}\right)(I+2 \pi \nu \mathcal{G})+\nu \mathcal{B}, \quad \mathcal{E} \equiv \frac{1}{K} e e^{t} .
$$

Since $\mathcal{B}_{j j} \equiv \hat{B}_{j}\left(\lambda, D, S_{j}\right)$ (cf. (3.21)), the eigenvalues $\lambda$ are obtained by seeking nontrivial solutions $c$ of (3.28) that $\operatorname{occur}$ when $\operatorname{Det}(\mathcal{A})=0$.

It is beyond the scope of this paper to provide a detailed numerical study of this non-standard eigenvalue problem for a fixed small value of $\nu \equiv-1 / \log \varepsilon$. However, to leading order in $\nu,(3.28)$ reduces to

$$
(I-\mathcal{D}) c=0, \quad \mathcal{D} \equiv\left(\frac{K}{e^{t} \mathcal{B}^{-1} e}\right) \mathcal{E B}^{-1}
$$

Clearly $\mathcal{D}$ is a rank-one matrix with $\mathcal{D} e=e$. Therefore, we conclude that for $\nu \ll 1, c=\alpha e$ for some constant $\alpha$. The constraint that $e^{t} c=0$ then enforces that $\alpha=0$, and consequently that $c=0$. Hence, $C_{j}=0$ for $j=1, \ldots, K$.

This leads to the important conclusion that, to leading order in $\nu$, the eigenvalue for the $m=0$ mode is obtained by solving $(3.20 a)$ subject to the condition that $N_{j}$ is bounded as $\rho \rightarrow \infty$. This was precisely the eigenvalue problem studied numerically in $\S 2.3$ for the case of a one-spot solution. However, this decoupling property for the stability problem does not occur for the case where $D$ is asymptotically large with $D=\mathcal{O}\left(\nu^{-1}\right)$. In this limiting regime, we show in $\S 6$ that (3.20) and (3.26) reduces to the vectorial nonlocal eigenvalue problem of [54].

In summary, for $D=\mathcal{O}(1)$ we conclude that to leading order in $\nu$ a $K$-spot pattern with $K>1$ is stable with respect to the $m=0$ mode, but will become unstable to the local $m=2$ mode when, for at least one value of $j, S_{j}$ exceeds the threshold value $\Sigma_{2} \approx 4.3$. This leads to the following spot-splitting criterion:

Spot-Splitting Criterion: Let $D=\mathcal{O}(1)$ and $\varepsilon \rightarrow 0$ and consider a $K$-spot quasi-equilibrium solution to (1.2). Let $S_{j}$ for $j=1, \ldots, K$, satisfy the nonlinear algebraic system (3.6) when $K>1$. For $K=1$, $S_{1}$ is given in (2.6). To leading order in $\nu$, a $K$-spot solution with $K>1$ is stable to the local angular mode $m=0$, whereas a one-spot solution is unconditionally stable to the $m=0$ mode. For $K \geq 1$ the quasi-equilibrium solution is stable with respect to the other local angular modes $m=2,3,4, \ldots$ provided that $S_{j}<\Sigma_{2} \approx 4.303$ for all $j=1, \ldots, K$. The $J^{\text {th }}$ spot will become unstable to the $m=2$ mode if $S_{J}$ exceeds the threshold value $\Sigma_{2}$. This peanut-splitting instability from the linearized problem is found to initiate a nonlinear spot self-replication process. 
We now illustrate our prediction for spot-splitting with regards to pattern formation on a slowly growing domain. Let $\varepsilon \ll 1, D$, and $a$, be fixed parameters. Suppose that $\Omega=[0, L] \times[0, L]$ is a square with side length $L=L(t)$ that grows slowly in time with growth rate $L^{\prime} \ll \mathcal{O}\left(\varepsilon^{2}\right)$, so that the domain growth is quasi-steady relative to the dynamics of spot motion. Then, from the Spot-Splitting Criterion above, we conclude that a one-spot quasi-equilibrium solution will begin to undergo self-replication when $S>\Sigma_{2} \approx 4.3$. By using (2.6) for $S$, and noting that $|\Omega|=L^{2}$, we solve for $L$ to obtain that splitting is initiated when $L>L_{1}$, where

$$
L_{1}=\left(\frac{2 \pi \sqrt{D} \Sigma_{2}}{a}\right)^{1 / 2}
$$

Moreover, to leading order in $\nu$ for $\nu \ll 1$, we conclude from the expression for $S_{j}$ in (3.13) that a $K$-spot quasiequilibrium solution will begin to self-replicate into a $2 K$-spot pattern when $L>L_{K} \equiv L_{1} \sqrt{K}$.

\section{The Neumann Green's Function}

The asymptotic results in $\S 2$ and $\S 3$ rely on detailed knowledge of the Neumann Green's function $G$ and its regular part $R$ satisfying (2.7). We now give some properties of these functions for both the unit disk and a rectangle.

\subsection{The Neumann Green's Function for the Unit Disk}

Let $\Omega:=\{x|| x \mid \leq 1\}$ be the unit disk and represent the point $x \in \Omega$ as a complex number. Then, from equation (4.3) of [26], the Neumann Green's function and its regular part are given explicitly by

$$
\begin{aligned}
& G(x ; \xi)=\frac{1}{2 \pi}\left(-\log |x-\xi|-\log |x| \xi\left|-\frac{\xi}{|\xi|}\right|+\frac{1}{2}\left(|x|^{2}+|\xi|^{2}\right)-\frac{3}{4}\right) \\
& R(\xi ; \xi)=\frac{1}{2 \pi}\left(-\log |\xi| \xi\left|-\frac{\xi}{|\xi|}\right|+|\xi|^{2}-\frac{3}{4}\right) .
\end{aligned}
$$

A simple calculation then yields

$$
\nabla G(x ; \xi)=-\frac{1}{2 \pi}\left[\frac{(x-\xi)}{|x-\xi|^{2}}+\frac{|\xi|^{2}}{\bar{x}|\xi|^{2}-\bar{\xi}}-x\right], \quad \nabla R(\xi ; \xi)=\frac{1}{2 \pi}\left(\frac{2-|\xi|^{2}}{1-|\xi|^{2}}\right) \xi,
$$

where ${ }^{-}$denotes complex conjugate. By substituting (4.2) in (3.17) of Principal Result 3.4, we can obtain explicit ODE's for the spots dynamics in terms of the source strengths $S_{j}, j=1, \ldots, K$.

\subsection{The Neumann Green's Function for a Rectangle}

In the rectangular domain $\Omega \equiv[0, L] \times[0, d]$, we now calculate the Neumann Green's function $G\left(x ; x^{\prime}\right)$ satisfying (2.7), where $x^{\prime}=\left(x^{\prime}, y^{\prime}\right)$ is located strictly inside $\Omega$. In this sub-section $x \in \Omega$ is written in the cartesian coordinates $x=(x, y)$. The Fourier series representation of $G\left(x ; x^{\prime}\right)$ is

$$
\begin{aligned}
G\left(x ; x^{\prime}\right)=\frac{2}{|\Omega|} \sum_{n=1}^{\infty} \frac{\cos \left(\frac{n \pi x}{L}\right) \cos \left(\frac{n \pi x^{\prime}}{L}\right)}{\left(\frac{n \pi}{L}\right)^{2}}+\frac{2}{|\Omega|} \sum_{m=1}^{\infty} \frac{\cos \left(\frac{m \pi y}{d}\right) \cos \left(\frac{m \pi y^{\prime}}{d}\right)}{\left(\frac{m \pi}{d}\right)^{2}} & \\
& +\frac{4}{|\Omega|} \sum_{m=1}^{\infty} \sum_{n=1}^{\infty} \frac{\cos \left(\frac{n \pi x}{L}\right) \cos \left(\frac{n \pi x^{\prime}}{L}\right) \cos \left(\frac{m \pi y}{d}\right) \cos \left(\frac{m \pi y^{\prime}}{d}\right)}{\left(\frac{n \pi}{L}\right)^{2}+\left(\frac{m \pi}{d}\right)^{2}},
\end{aligned}
$$


where $|\Omega|=L d$. Clearly, the constraint $\int_{\Omega} G d x=0$ is satisfied. Upon recalling the identity (cf. page 45 of $[\mathbf{2 0}]$ )

$$
\sum_{k=1}^{\infty} \frac{\cos (k \pi \theta)}{k^{2}}=\left(\frac{\pi^{2}}{12}\right) h(\theta), \quad h(\theta) \equiv 2-6|\theta|+3 \theta^{2}, \quad|\theta| \leq 2,
$$

and by using the angle addition formula for cosine, we can sum the first term in (4.3) to obtain

$$
\frac{2}{|\Omega|} \sum_{n=1}^{\infty} \frac{\cos \left(\frac{n \pi x}{L}\right) \cos \left(\frac{n \pi x^{\prime}}{L}\right)}{\left(\frac{n \pi}{L}\right)^{2}}=\frac{1}{d} H\left(x, x^{\prime}\right), \quad H\left(x, x^{\prime}\right) \equiv \frac{L}{12}\left[h\left(\frac{x-x^{\prime}}{L}\right)+h\left(\frac{x+x^{\prime}}{L}\right)\right] .
$$

The function $H\left(x ; x^{\prime}\right)$ is precisely the one-dimensional Green's function in the horizontal $x$-direction.

Next, upon recalling the formula (cf. page 46 of [20])

$$
\sum_{k=1}^{\infty} \frac{\cos (k \pi \theta)}{k^{2}+b^{2}}=\frac{\pi}{2 b}\left(\frac{\cosh (b \pi(1-|\theta|))}{\sinh (b \pi)}\right)-\frac{1}{2 b^{2}}, \quad|\theta| \leq 2,
$$

we can sum the third term of (4.3) over the index $n$ to obtain

$$
\frac{1}{2 \pi} \sum_{m=1}^{\infty}\left[\cos \left(\frac{m \pi}{d}\left(y-y^{\prime}\right)\right)+\cos \left(\frac{m \pi}{d}\left(y+y^{\prime}\right)\right)\right] \frac{F_{m}\left(x, x^{\prime}\right)}{m}-\frac{2}{|\Omega|} \sum_{m=1}^{\infty} \frac{\cos \left(\frac{m \pi y}{d}\right) \cos \left(\frac{m \pi y^{\prime}}{d}\right)}{\left(\frac{m \pi}{d}\right)^{2}} .
$$

Here $F_{m}\left(x, x^{\prime}\right)$ is defined by

$$
F_{m}\left(x, x^{\prime}\right) \equiv \frac{\cosh \left[\frac{m L \pi}{d}\left(1-\frac{\left|x-x^{\prime}\right|}{L}\right)\right]+\cosh \left[\frac{m L \pi}{d}\left(1-\frac{\left|x+x^{\prime}\right|}{L}\right)\right]}{\sinh \left(\frac{m L \pi}{d}\right)} .
$$

Therefore, upon substituting (4.6) and (4.4 b) into (4.3), we obtain that the second sum in (4.3) cancels so that

$$
G\left(x ; x^{\prime}\right)=\frac{1}{d} H\left(x, x^{\prime}\right)+\frac{1}{2 \pi} \sum_{m=1}^{\infty}\left[\cos \left(\frac{m \pi\left(y-y^{\prime}\right)}{d}\right)+\cos \left(\frac{m \pi\left(y+y^{\prime}\right)}{d}\right)\right] \frac{F_{m}\left(x, x^{\prime}\right)}{m} .
$$

Next we rewrite $F_{m}\left(x, x^{\prime}\right)$ in (4.7) by using the following identity that holds for any constants $a, b$, and $c$ :

$$
\frac{\cosh (a-b)+\cosh (a-c)}{\sinh a}=\frac{1}{1-\mathrm{e}^{-2 a}}\left[\mathrm{e}^{-b}+\mathrm{e}^{-c}+\mathrm{e}^{b-2 a}+\mathrm{e}^{c-2 a}\right] .
$$

Then, we use (4.9), together with the identities $\cos (\theta)=\operatorname{Re}\left(e^{i \theta}\right)$ for $\theta$ real and $\left(1-q^{m}\right)^{-1}=\sum_{n=0}^{\infty}\left(q^{m}\right)^{n}$ whenever $|q|<1$. In this way, the infinite sum in (4.8) can be written compactly as the doubly infinite sum

$$
\frac{1}{2 \pi} \operatorname{Re}\left[\sum_{m=1}^{\infty} \sum_{n=0}^{\infty} \frac{\left(q^{n}\right)^{m}}{m}\left(z_{+,+}^{m}+z_{+,-}^{m}+z_{-,+}^{m}+z_{-,-}^{m}+\zeta_{+,+}^{m}+\zeta_{+,-}^{m}+\zeta_{-,+}^{m}+\zeta_{-,-}^{m}\right)\right],
$$

where the eight complex constants $z_{ \pm, \pm}, \zeta_{ \pm, \pm}$are defined in terms of additional complex constants $r_{ \pm, \pm}, \rho_{ \pm, \pm}$by

$$
\begin{aligned}
& z_{ \pm, \pm} \equiv \mathrm{e}^{\mu r_{ \pm, \pm} / 2}, \quad \zeta_{ \pm, \pm} \equiv \mathrm{e}^{\mu \rho_{ \pm, \pm} / 2}, \quad \mu \equiv \frac{2 \pi}{d} \quad q \equiv \mathrm{e}^{-\mu L}<1, \\
& r_{+, \pm} \equiv-\left|x+x^{\prime}\right|+i\left(y \pm y^{\prime}\right), \quad r_{-, \pm} \equiv-\left|x-x^{\prime}\right|+i\left(y \pm y^{\prime}\right), \\
& \rho_{+, \pm} \equiv\left|x+x^{\prime}\right|-2 L+i\left(y \pm y^{\prime}\right), \quad \rho_{-, \pm} \equiv\left|x-x^{\prime}\right|-2 L+i\left(y \pm y^{\prime}\right) .
\end{aligned}
$$

The doubly infinite sum in (4.10) is absolutely convergent provided that $z_{ \pm, \pm} \neq 1$ and $\zeta_{ \pm, \pm} \neq 1$. Under this condition, we can interchange the order of summation in (4.10) and then perform the sum over the index $m$ by using 
the identity $\operatorname{Re}\left[\sum_{m=1}^{\infty} m^{-1} \omega^{m}\right]=-\log |1-\omega|$ for $|\omega|<1$. In this way, (4.10) becomes

$$
\begin{aligned}
-\frac{1}{2 \pi} \sum_{n=0}^{\infty} \log \left(\mid 1-q^{n} z_{+,+}\left\|1-q^{n} z_{+,-}\right\| 1\right. & \left.-q^{n} z_{-,+} \| 1-q^{n} z_{-,-}\right) \\
& -\frac{1}{2 \pi} \sum_{n=0}^{\infty} \log \left(\mid 1-q^{n} \zeta_{+,+}\left\|1-q^{n} \zeta_{+,-}\right\| 1-q^{n} \zeta_{-,+} \| 1-q^{n} \zeta_{-,-}\right) .
\end{aligned}
$$

The only singularity exhibited by (4.12) in $\Omega$ is at $(x, y)=\left(x^{\prime}, y^{\prime}\right)$, in which case $z_{-,-}=1$ and $\log \left|1-z_{-,-}\right|$diverges. We then write $\log \left|1-z_{-,-}\right|=\log \left|r_{-,-}\right|+\log \left(\left|1-z_{-,-}\right| /\left|r_{-,-}\right|\right)$, and note that $\log \left|r_{-,-}\right|=\log \left|x-x^{\prime}\right|$ and that $\log \left(\left|1-z_{-,-}\right| /\left|r_{-,-}\right|\right)$is finite at $x=x^{\prime}$. Finally, upon using (4.12) to replace the sum in (4.8), and upon extracting the singular term in (4.12), we readily obtain that (4.8) reduces to

$$
G\left(x ; x^{\prime}\right)=-\frac{1}{2 \pi} \log \left|x-x^{\prime}\right|+R\left(x ; x^{\prime}\right),
$$

where the regular part $R\left(x ; x^{\prime}\right)$ is given explicitly by

$$
\begin{array}{r}
R\left(x ; x^{\prime}\right)=-\frac{1}{2 \pi} \sum_{n=0}^{\infty} \log \left(\left|1-q^{n} z_{+,+}\left\|1-q^{n} z_{+,-}\right\| 1-q^{n} z_{-,+}\right|\left|1-q^{n} \zeta_{+,+}\left\|1-q^{n} \zeta_{+,-}\right\| 1-q^{n} \zeta_{-,+} \| 1-q^{n} \zeta_{-,-}\right|\right) \\
-\frac{1}{2 \pi} \log \frac{\left|1-z_{-,-}\right|}{\left|r_{-,-}\right|}+\frac{1}{d} H\left(x, x^{\prime}\right)-\frac{1}{2 \pi} \sum_{n=1}^{\infty} \log \left|1-q^{n} z_{-,-}\right| .
\end{array}
$$

In $(4.13 b), H\left(x, x^{\prime}\right)$ is defined in (4.4), and the complex constants $z_{ \pm, \pm}, \zeta_{ \pm, \pm}, r_{-,-}$and the real constant $q<1$ are defined in (4.11). Finally, we calculate the self-interaction term $R\left(x^{\prime} ; x^{\prime}\right)$ by taking the limit $x \rightarrow x^{\prime}$ in $(4.13 b)$. A simple calculation using $(4.13 b),(4.4)$, and $(4.11)$, yields the exact formula

$$
\begin{array}{r}
R\left(x^{\prime} ; x^{\prime}\right)=-\frac{1}{2 \pi} \sum_{n=0}^{\infty} \log \left(\left|1-q^{n} z_{+,+}^{0}\left\|1-q^{n} z_{+,-}^{0}\right\| 1-q^{n} z_{-,+}^{0}\left\|1-q^{n} \zeta_{+,+}^{0}\right\| 1-q^{n} \zeta_{+,-}^{0}\left\|1-q^{n} \zeta_{-,+}^{0}\right\| 1-q^{n} \zeta_{-,-}\right|\right) \\
+\frac{L}{d}\left(\frac{1}{3}-\frac{x^{\prime}}{L}+\left(\frac{x^{\prime}}{L}\right)^{2}\right)-\frac{1}{2 \pi} \log \left(\frac{\pi}{d}\right)-\frac{1}{2 \pi} \sum_{n=1}^{\infty} \log \left(1-q^{n}\right), \quad(4.14
\end{array}
$$

where $q \equiv e^{-2 L \pi / d}$. Here $z_{ \pm, \pm}^{0}$ and $\zeta_{ \pm, \pm}^{0}$ are the limits of $z_{ \pm, \pm}$and $\zeta_{ \pm, \pm}$as $x \rightarrow x^{\prime}=\left(x^{\prime}, y^{\prime}\right)$ given explicitly by

$$
\begin{aligned}
& z_{+,+}^{0}=\mathrm{e}^{\mu\left(-x^{\prime}+i y^{\prime}\right)}, \quad z_{+,-}^{0}=\mathrm{e}^{-\mu x^{\prime}}, \quad z_{-,+}^{0}=\mathrm{e}^{\mu i y^{\prime}}, \quad \mu \equiv 2 \pi / d, \\
& \zeta_{+,+}^{0}=\mathrm{e}^{\mu\left(x^{\prime}-L+i y^{\prime}\right)}, \quad \zeta_{-,+}^{0}=\mathrm{e}^{\mu\left(-L+i y^{\prime}\right)}, \quad \zeta_{+,-}^{0}=\mathrm{e}^{\mu\left(x^{\prime}-L\right)}, \quad \zeta_{-,-}^{0}=\mathrm{e}^{-\mu L} .
\end{aligned}
$$

We can assume that $L \geq d$, since otherwise we can interchange $x$ and $y$. Therefore, $q \leq e^{-2 \pi}$ and $q^{n}$ rapidly tends to zero, leading to rapidly converging infinite series in (4.14) and (4.13). Therefore, even for the unit square where $L=d=1$ and $q=\mathrm{e}^{-2 \pi}$, in practice the infinite sums in (4.14) and (4.13) can be approximated to a high degree of accuracy by retaining only a few terms in each sum. This rapid convergence of the representation (4.14) and (4.13) is in direct contrast to the poor convergence property of the Fourier series representation (4.3). In addition, we remark that we can also readily calculate $\nabla R\left(x^{\prime} ; x^{\prime}\right)$ and $\nabla G\left(x ; x^{\prime}\right)$, which are needed for the slow dynamics in (3.17). We also remark that a partial summation of (4.3) was given previously in [32] and [33]. Analogous infinite source term representations for a modified Green's function satisfying different boundary conditions than in (4.3) was given in [8] in the study of the diffusion of protein receptors on a dendritic cable in the presence of traps. 


\section{Comparison of the Asymptotic Theory with Full Numerical Simulations}

In this section we compare full numerical results computed from (1.2) with those obtained from numerically solving the asymptotic DAE system (3.6) and (3.17) that describes the dynamics of a $K$-spot quasi-equilibrium pattern. We will consider different initial configurations $x_{1}, \ldots, x_{K}$ of spot locations inside either a square domain or the unit disk, and we will test the Spot-Splitting Criterion of $\S 3$.

The asymptotic DAE system is solved numerically by using Newton's method to solve the nonlinear algebraic system (3.6) and a Runge-Kutta ODE solver to evolve the dynamics (3.17). We emphasize that there are exactly two functions $\gamma(S)$ and $\chi(S)$ in the DAE system that depend on the nonlinear (inner) core problem. These functions are pre-computed at 100 grid points in $S$ and a cubic spline interpolation is fitted to the discretely sampled functions in order to determine them at an arbitrary value of $S$. For the unit disk or a square domain, the Green's functions $G$ and $R$, together with their gradients, which are required in the DAE system, can be calculated by using the results in $\S 4$. The results obtained in this way are referred to below as the "asymptotic" results.

In computing full numerical solutions to (1.2) we have used several different codes. The adaptive-grid finite difference solver VLUGR2 (cf. [6]) was used to compute numerical solutions of (1.2) in the square, while the finite element solver of [27] was used to compute solutions in the unit disk. Unless otherwise stated below, the initial condition for (1.2) is chosen as

$$
v=\sqrt{D} \sum_{j=1}^{K} v_{j} \operatorname{sech}^{2}\left(\frac{\left|x-x_{j}\right|}{2 \varepsilon}\right), \quad u=-\frac{2 \pi}{\sqrt{D}}\left(\sum_{j=1}^{K} S_{j} G\left(x ; x_{j}\right)+u_{c}\right),
$$

where a cut-off was imposed for $G$ whenever $\left|x-x_{j}\right| \leq \varepsilon$. Here $S_{j}$, for $j=1, \ldots, N$, and $u_{c}$ is obtained from the numerical solution to (3.6) and (3.17) for the initial spot configuration $x_{1}, \ldots, x_{K}$. In addition, $v_{j}=V_{j}(0)$, where $V_{j}(\rho)$ is the radially symmetric solution of the core problem. In terms of the numerical solution to (1.2), we track the time-dependent spot locations by identifying points in $\Omega$ where $v$ has $\mathcal{O}(1)$ local maxima on the computational grid. We did not attempt to determine more accurate interpolated local maxima by fitting a bi-cubic spline to points in a neighborhood of this discrete local maximum. As a consequence our numerical results for the spot trajectories have a small degree of raggedness.

Experiment 1 (Slow Drift of One Spot): Let $\Omega:=\{x|| x \mid \leq 1\}$. For this case, the dynamics of the spot is given by (2.21) where $\nabla R\left(x_{0} ; x_{0}\right)$ was given in (4.2) and $S$ was given in (2.6). This gives the ODE

$$
\frac{d x_{0}}{d t} \sim-\varepsilon^{2} S \gamma(S)\left(\frac{2-\left|x_{0}\right|^{2}}{1-\left|x_{0}\right|^{2}}\right) x_{0}, \quad S=\frac{a}{2 \sqrt{D}} .
$$

We fix $\varepsilon=0.03, a=6.6$, and $D=1$, so that $S=3.3$. Since $S=3.3<\Sigma_{2} \approx 4.3$, the asymptotic theory predicts that the spot should slowly drift towards the origin without splitting. The initial spot location is $x_{0}=(0.5,0)$. In Fig. 7(a) we plot the distance $\left|x_{0}\right|$ of the spot to the origin as a function of time as obtained from the ODE (5.2) (solid curve) and the full numerical solution of (1.2) (discrete points). The asymptotic results agree very closely with the full numerical results.

Next, we let $\Omega$ to be the unit square with an initial spot located at $x_{0}=(0.2,0.8)$ with $\varepsilon=0.02, a=8.25$, and $D=0.1$. For this case, the source strength $S=4.15$ is slightly below the spot-splitting threshold. In Fig. 7(b) we show a very close agreement between the asymptotic and full numerical results for the dynamics of the distance of the spot to the center of the square. 


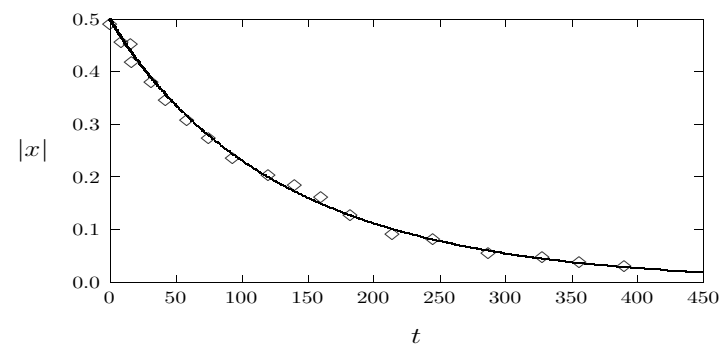

(a) $\left|x_{0}\right|$ vs. $t$

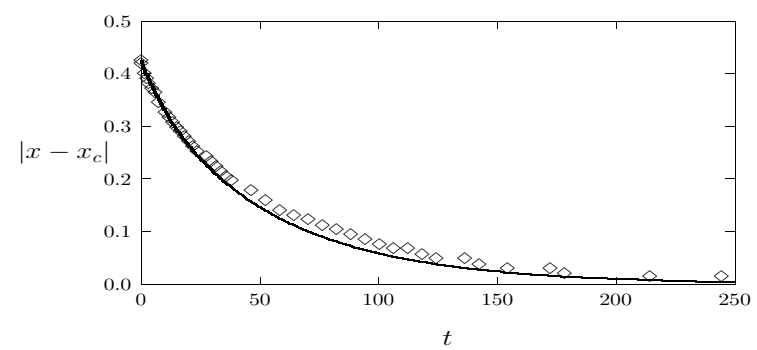

(b) $\left|x_{0}-x_{c}\right|$ vs. $t$

FIgURE 7. Left figure: plot of the distance $\left|x_{0}\right|$ to the origin vs. $t$ for an initial spot at $x_{0}=(0.5,0)$ in the unit disk with $\varepsilon=0.03, a=6.6$, and $D=1$. Right figure: plot of the distance $\left|x_{0}-x_{c}\right|$ of the spot to the center $x_{c}=(0.5,0.5)$ of the unit square vs. $t$ for an initial spot at $x_{0}=(0.2,0.8)$ with $\varepsilon=0.02, a=8.25$, and $D=0.1$. In these figures the solid curves are the asymptotic results and the discrete points are obtained from the full numerical solution of (1.2).

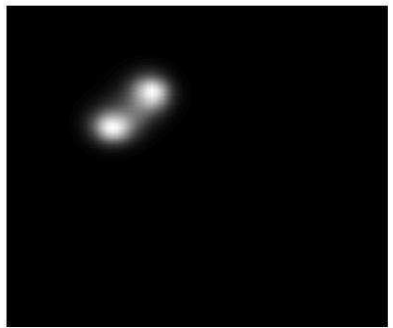

(a) $t=23.6$

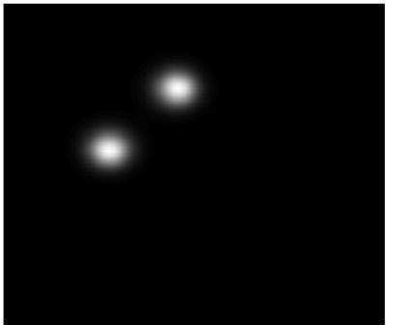

(b) $t=40.2$

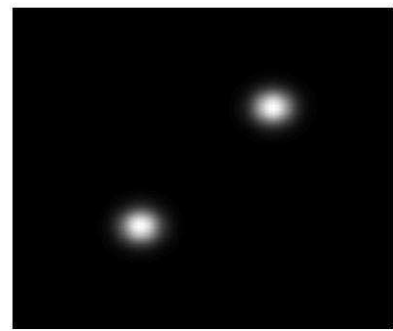

(c) $t=322.7$

FiguRE 8. Grayscale plot of $v$ computed from (1.2) for $\varepsilon=0.02, a=10, D=0.1$, for an initial one-spot pattern in the unit square with initial spot location $x_{0}=(0.2,0.8)$. The spot-splitting event leads to a two-spot equilibrium state.

Experiment 2 (The Splitting of One Spot): Next, we consider an initial one-spot quasi-equilibrium solution in the unit square for $\varepsilon=0.02, a=10$, and $D=0.1$. The initial spot location is $x_{0}=(0.2,0.8)$. For this case, we calculate from $(2.6)$ that $S \approx 5.03$. Since $S>\Sigma_{2} \approx 4.3$, we predict that the spot will undergo splitting starting at $t=0$. This is confirmed in Fig. 8 where we plot $v$ at several values of $t$. From Fig. 4(a) the growth rate of the initial peanut-splitting instability is $\lambda_{0}(S, 2) \approx 0.15$ when $S \approx 5.03$. As $t$ increases, the two-spot pattern converges to an equilibrium solution with spots at $x_{1 e} \approx(0.322,0.318)$ and $x_{2 e} \approx(0.677,0.682)$ (see Fig. 8(c)).

Next, we test the sharpness of the asymptotic instability threshold $\Sigma_{2} \approx 4.3$ for $\varepsilon=0.02$. Since $S$ is independent of the spot location, the asymptotic theory predicts that the occurrence of spot-splitting is independent of the initial spot location. By fixing $\varepsilon=0.02$ and $D=0.1$, and then solving (1.2) numerically for different values of $a$ and initial spot location in the unit square we obtain the following results:

$$
\begin{aligned}
& x_{0}=(0.2,0.8), \quad a=8.5, \quad S \approx 4.28, \quad \text { Splitting Observed, } \quad x_{1 e} \approx(0.322,0.318), \quad x_{2 e} \approx(0.677,0.682), \\
& x_{0}=(0.2,0.8), \quad a=8.25, \quad S \approx 4.15, \quad \text { No Splitting Observed, } \quad x_{1 e}=(0.5,0.5), \\
& x_{0}=(0.7,0.4), \quad a=8.25, \quad S \approx 4.15, \quad \text { No Splitting Observed, } \quad x_{1 e}=(0.5,0.5), \\
& x_{0}=(0.7,0.4), \quad a=8.5, \quad S \approx 4.28, \quad \text { Splitting Observed, } \quad x_{1 e} \approx(0.354,0.292), \quad x_{2 e} \approx(0.646,0.708) .
\end{aligned}
$$


The last entry in each of the rows is the computed equilibrium locations. Therefore, for $\varepsilon=0.02$, the numerically predicted instability threshold is between 4.15 and 4.28 , which is rather close to the asymptotic result of $\Sigma_{2} \approx 4.3$.

Experiment 3 (Two-Spot Patterns in a Square): Next we consider instabilities of two-spot solutions in the unit square. In the four experiments below we have fixed $\varepsilon=0.02, D=0.1$, and have chosen $x_{1}=(0.3,0.3)$ to be the initial location of one of the spots. We will vary only $a$ and the initial location $x_{2}$ of the second spot.

First we take $a=15$ and set $x_{2}=(0.5,0.8)$ at $t=0$. For this case, we calculate from (3.6) that $S_{1} \approx 3.83$ and $S_{2} \approx 3.72$, which are both below the instability threshold. In the first row of Fig. 9 we plot $v$ at several values of $t$ showing a slow drift of the spot locations towards their equilibrium values at $x_{1 e} \approx(0.32,0.32)$ and $x_{2 e} \approx(0.68,0.68)$. In the first row of Fig. 10 we show a very favorable comparison between the asymptotic and full numerical results for the $x$ and $y$ coordinates of the two spot locations versus time.

Next, we increase $a$ to $a=18$ and again set $x_{2}=(0.5,0.8)$ at $t=0$. For this case, we calculate from (3.6) that $S_{1} \approx 4.60$ and $S_{2} \approx 4.46$, which are both above the instability threshold of $\Sigma_{2} \approx 4.3$. Therefore, the asymptotic theory predicts that both spots will begin a splitting process at $t=0$. The full numerical results in the second row of Fig. 9 show two splitting events and an eventual four-spot equilibrium solution as $t$ increases. The DAE system (3.6) and (3.17) is not valid during a spot-splitting event. However, if we choose the initial conditions for (3.17) to be the spot locations from the full numerical solution at some time slightly after the splitting has occurred, then as shown in the second row of Fig. 10 the asymptotic results for the spot trajectories compare very favorably with the full numerical results at subsequent times. For Fig. 10(c) and Fig. 10(d) this calibration of the initial condition for (3.17) was done at $t \approx 40$. The final four-spot equilibrium solution has spots at $x_{1 e} \approx(0.25,0.25), x_{2 e} \approx(0.75,0.25)$, $x_{3 e}=(0.25,0.75)$, and $x_{4 e} \approx(0.75,0.75)$, with $S_{j} \approx 2.265$ for $j=1, \ldots, 4$.

For our third example we again set $a=18$ but now take $x_{2}=(0.8,0.8)$. For this case, we calculate from (3.6) that $S_{1} \approx 5.27$ and $S_{2} \approx 3.79$. Since $S_{1}>\Sigma_{2}$, the asymptotic theory predicts that only the spot at $x_{1}$ will split. This asymptotic prediction is confirmed from the full numerical results shown in the third row of Fig. 9. The initial locations for the asymptotic spot dynamics (3.17) are calibrated from the full numerical results at $t=20$. In the third row of Fig. 10 we show that asymptotic predictions for the two cartesian coordinates of the three spot locations compare very closely with corresponding full numerical results for $t \geq 20$. The asymptotic theory predicts an equilibrium three-spot solution with spots at $x_{1 e} \approx(0.21,0.59), x_{2 e} \approx(0.59,0.21)$, and $x_{3 e} \approx(0.73,0.73)$.

Finally, we keep $a=18$, but change the initial spot location $x_{2}$ to $x_{2}=(0.5,0.6)$. For this case, we calculate from (3.6) that $S_{1} \approx 3.67$ and $S_{2} \approx 5.39$. Therefore, in contrast to the previous example, we predict that only the spot at $x_{2}=(0.5,0.6)$ will split. The full numerical results in the fourth row of Fig. 9 again confirm this asymptotic prediction. As shown in the fourth row of Fig. 10, the asymptotic DAE system (3.6) and (3.17) also again accurately predicts the spot trajectories after the splitting event. The final three-spot equilibrium solution has spots at $x_{1 e} \approx(0.31,0.24)$, $x_{2 e} \approx(0.35,0.78)$, and $x_{3 e}=(0.79,0.47)$.

We emphasize that in the last three rows of Fig. 9 we have fixed $\varepsilon=0.02, a=18, D=0.1$, and $x_{1}=(0.3,0.3)$, and have only varied the initial location $x_{2}$ of the other spot. Our results have shown three different possible dynamical behaviors depending on our choice for $x_{2}$.

Experiment 4 (An Initial Four-Spot Pattern): Next we consider an initial four-spot pattern in the unit square when $\varepsilon=0.2, a=28$, and $D=0.1$. The initial spot locations $x_{j}$, for $j=1, \ldots, 4$, are taken to be equi-distributed on a circle of radius $r_{c}=0.2$ centered at $(0.6,0.6)$ and are labeled as $x_{1}=(0.6,0.8), x_{2}=(0.4,0.6), x_{3}=(0.6,0.4)$, and $x_{4}=(0.8,0.6)$. From (3.6) we compute numerically that $S_{1}=S_{4} \approx 2.34$ and $S_{2}=S_{3} \approx 4.71$. Therefore, since 


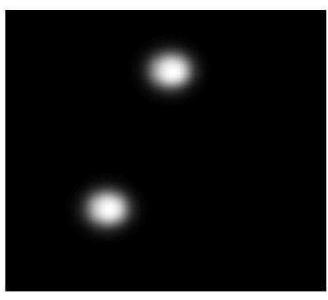

(a) $t=2.0$

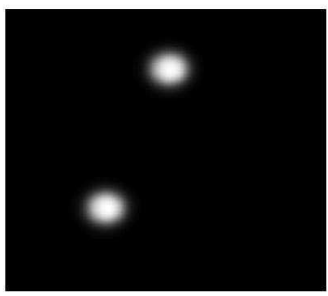

(e) $t=2.0$

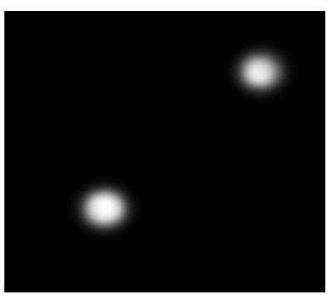

(i) $t=2.5$

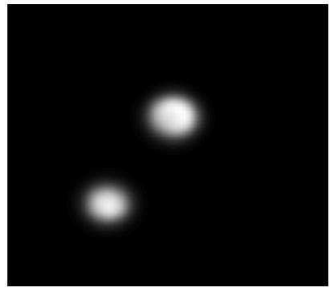

(m) $t=4.0$

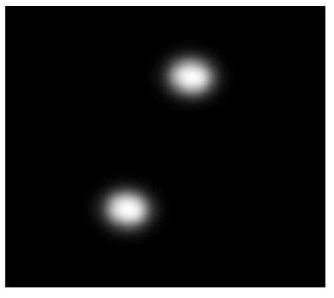

(b) $t=33.6$

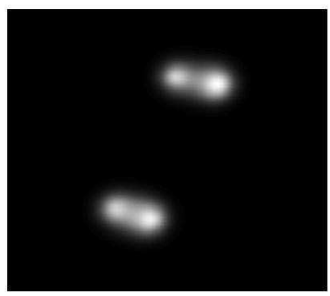

(f) $t=33.5$

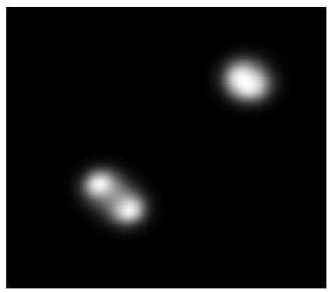

(j) $t=19.9$

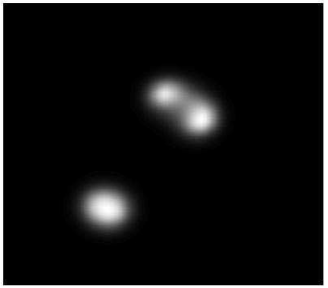

(n) $t=16.5$

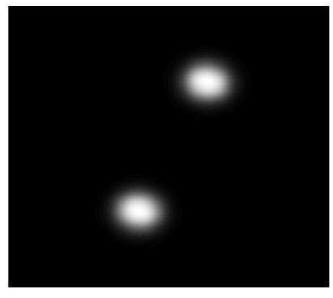

(c) $t=46.3$

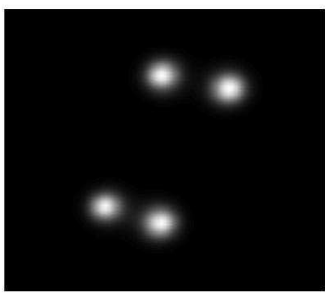

(g) $t=46.3$

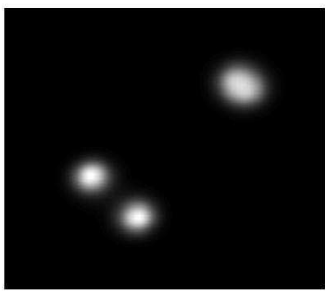

(k) $t=29.4$

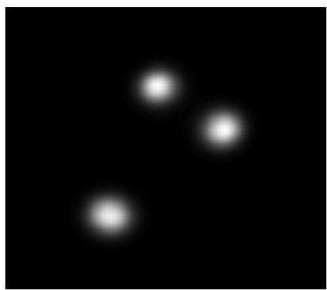

(o) $t=29.4$

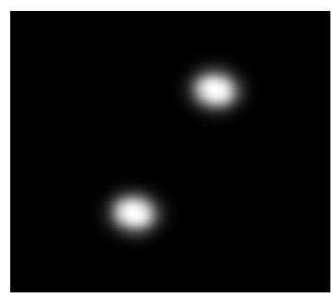

(d) $t=280.3$

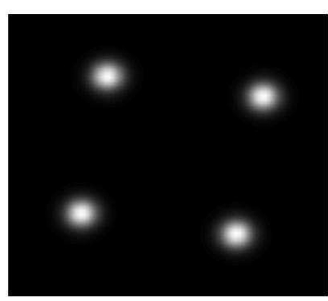

(h) $t=280.3$

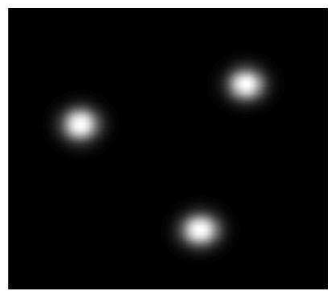

(l) $t=220.3$

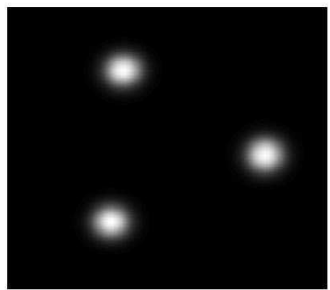

(p) $t=322.7$

FiguRE 9. Grayscale plots of $v$ for an initial two-spot quasi-equilibrium solution at different times for various parameter sets when $\Omega=[0,1] \times[0,1]$ with $\varepsilon=0.02, D=0.1$, and $x_{1}=(0.3,0.3)$. First Row: $a=15$ and $x_{2}=(0.5,0.8)$. No splitting occurs and there is a two-spot equilibrium solution. Second Row: $a=18$ and $x_{2}=(0.5,0.8)$. Both spots split, leading to a four-spot equilibrium solution. Third Row: $a=18$ and $x_{2}=(0.8,0.8)$. The spot at $x_{1}$ splits, and there is a three-spot equilibrium solution. Fourth Row: $a=18$ and $x_{2}=(0.5,0.6)$. The spot at $x_{2}$ splits, and there is a three-spot equilibrium solution. 


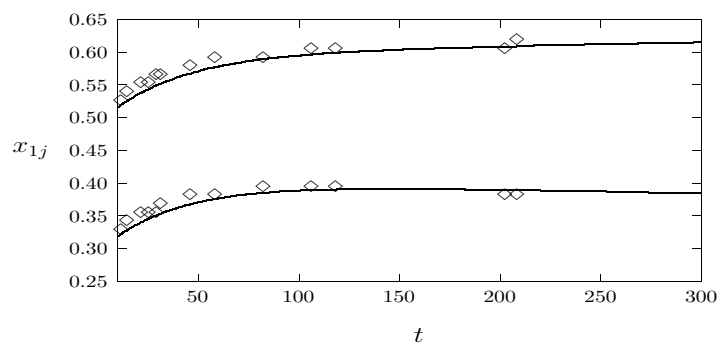

(a) $x_{j}$ vs. $t$

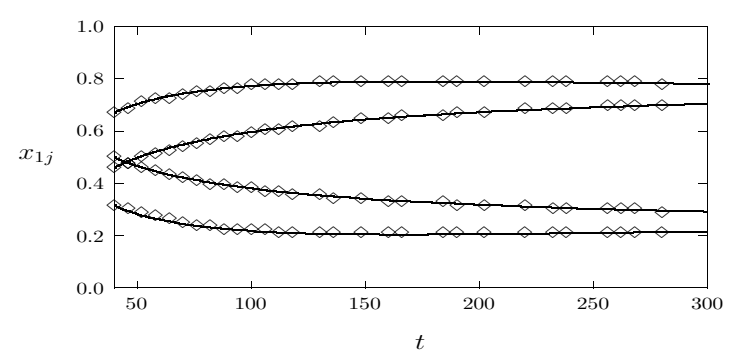

(c) $x_{j}$ vs. $t$

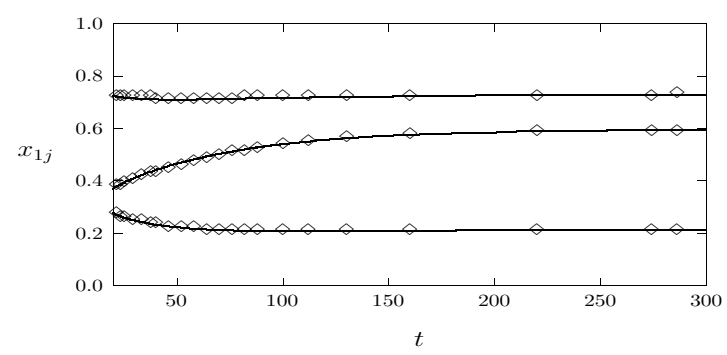

(e) $x_{j}$ vs. $t$

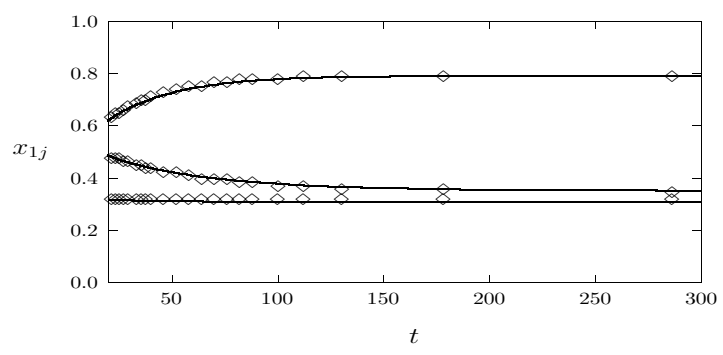

(g) $x_{j}$ vs. $t$

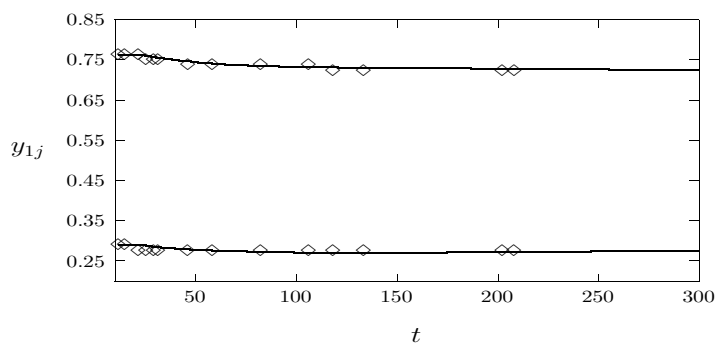

(b) $y_{j}$ vs. $t$

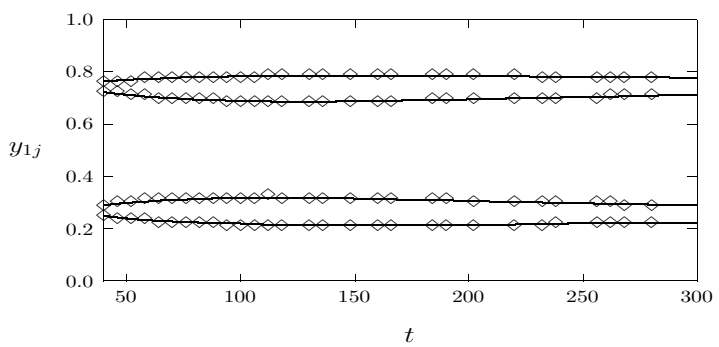

(d) $y_{j}$ vs. $t$

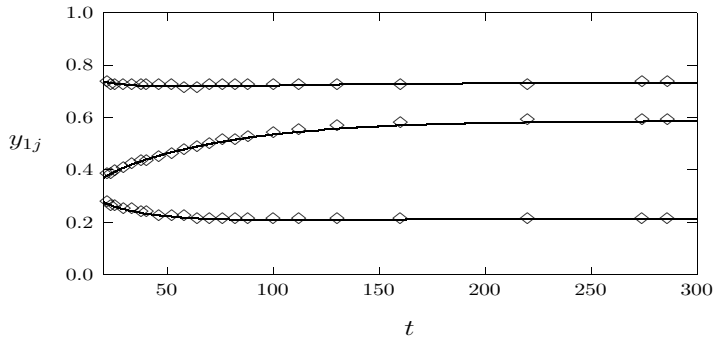

(f) $y_{j}$ vs. $t$

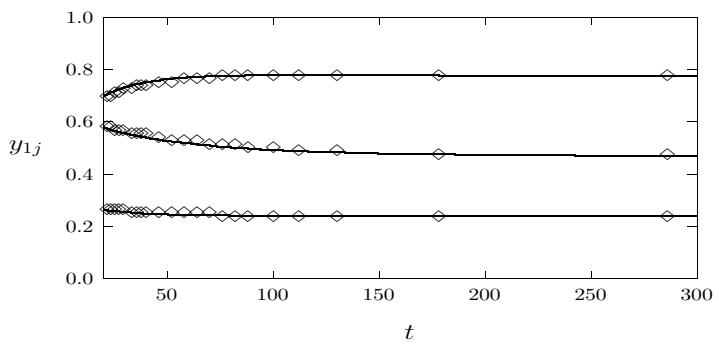

(h) $y_{j}$ vs. $t$

FiguRE 10. Comparison of the full numerical results for the two cartesian coordinates of the spot locations as computed numerically from (1.2) with corresponding results computed from the asymptotic DAE system (3.6) and (3.17) when $\Omega=$ $[0,1] \times[0,1], \varepsilon=0.02, D=0.1$, and $x_{1}=(0.3,0.3)$. The rows in this figure correspond to the rows in Fig. 9 . First Row: $a=15$ and $x_{2}=(0.5,0.8)$. Second Row: $a=18$ and $x_{2}=(0.5,0.8)$. The initial conditions for (3.17) calibrated from the full numerical results at $t \approx 40$. Third Row: $a=18$ and $x_{2}=(0.8,0.8)$. The initial conditions for $(3.17)$ calibrated from the full numerical results at $t \approx 20$. Fourth Row: $a=18$ and $x_{2}=(0.5,0.6)$. The initial conditions for $(3.17)$ calibrated from the full numerical results at $t \approx 20$. 

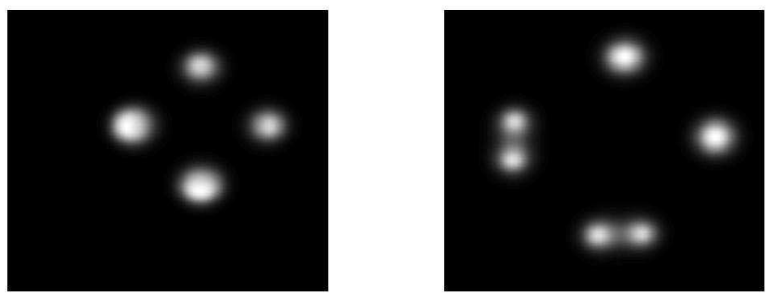

(a) $t=3.0$

(b) $t=64.3$

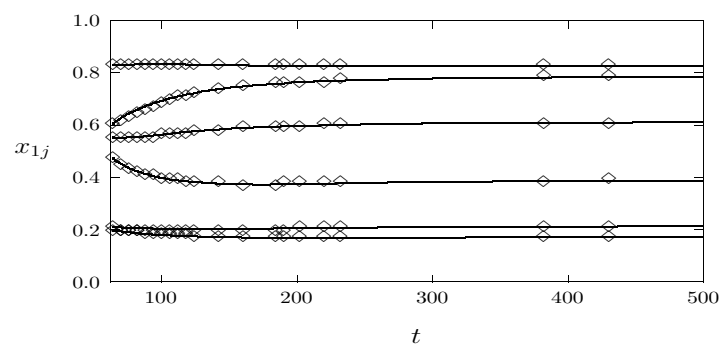

(e) $x_{j}$ vs. $t$
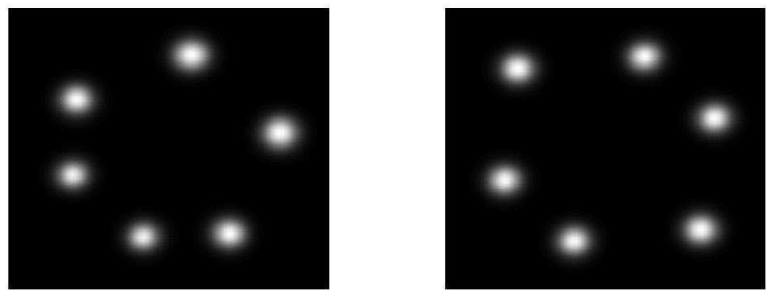

(c) $t=94.3$

(d) $t=430.3$

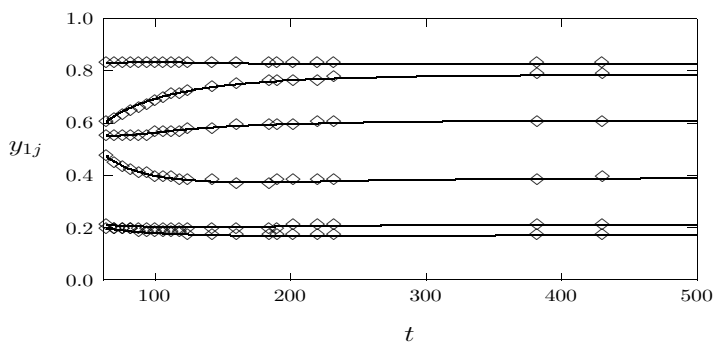

(f) $y_{j}$ vs. $t$

FiguRE 11. Grayscale plots of $v$ at different times together with the cartesian coordinates of the spot trajectories for an initial four-spot pattern with spots equi-distributed on a ring with center $(0.6,0.6)$ and radius $r_{c}=0.2$ in the unit square. The parameter values are $\varepsilon=0.02, a=28$, and $D=0.1$. Two of the spots split, leading to a six-spot equilibrium. The calibration between the asymptotic dynamics (3.17) (solid curves) and the full numerical results (discrete points) is done at $t \approx 64$.

$S_{2}=S_{3}>\Sigma_{2}$, we predict that only the spots at $x_{2}$ and $x_{3}$ will undergo self-replication. This is confirmed in Fig. 11 where we plot the numerical solution to (1.2) at different times, showing two splitting events and an eventual sixspot final equilibrium pattern. The six-spot pattern shown in Fig. 11(d) at $t \approx 430$, which closely approximates the equilibrium solution, is nearly hexagonal. In Fig. 11(e) and Fig. 11(f) we show a very favorable comparison, after the splitting has occurred, between the asymptotic spot trajectories computed from (3.6) and (3.17), and corresponding full numerical results computed from (1.2). The initial conditions for (3.17) were calibrated at $t \approx 64$ (see Fig. 11(b)).

Experiment 5 (An Initial Six-Spot Pattern): Our final example for the unit square concerns Fig. 1 of $\S 1$. We consider an initial six-spot pattern when $\varepsilon=0.02, a=51$, and $D=0.1$. The initial spots locations are equidistributed on a circle of radius $r_{c}=0.33$ centered at the midpoint $x_{c} \equiv(0.5,0.5)$ of the square. The initial spot locations $x_{j}$ for $j=1, \ldots, 6$, written as complex numbers, together with their corresponding source strengths as computed from (3.6), are

$$
x_{j}=x_{c}+r_{c} e^{i \pi(j-1) / 3}, \quad j=1, \ldots, 6 ; \quad S_{1}=S_{4} \approx 4.01, \quad S_{2}=S_{3}=S_{5}=S_{6} \approx 4.44 .
$$

Therefore, we predict that the spots located initially at $x_{2}, x_{3}, x_{5}$, and $x_{6}$ will split and that there will be a tenspot final equilibrium pattern. This is precisely what is observed in Fig. 1. From Fig. 4(a) the growth rate of the initial peanut-splitting instability is $\lambda_{0}(S, 2) \approx 0.025$ when $S \approx 4.44$. This agrees rather well with the time-scale for splitting as observed in the first row of Fig. 1. An interesting observation regarding Fig. 1 is that, although the initial six-spot quasi-equilibrium pattern is symmetric about the vertical line $x=0.5$ (see Fig. 1(a)), the final 
equilibrium state closely approximated by Fig. 1(f) does not have this symmetry. In Fig. 12 we show a very favorable comparison, after the splitting has occurred, between the asymptotic spot trajectories computed from (3.6) and (3.17) and corresponding results computed numerically from (1.2). The initial conditions for (3.17) were calibrated at $t \approx 70$.

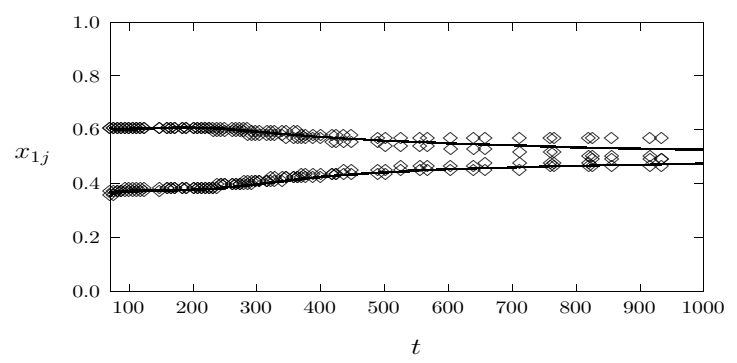

(a) $x_{j}$ vs. $t$

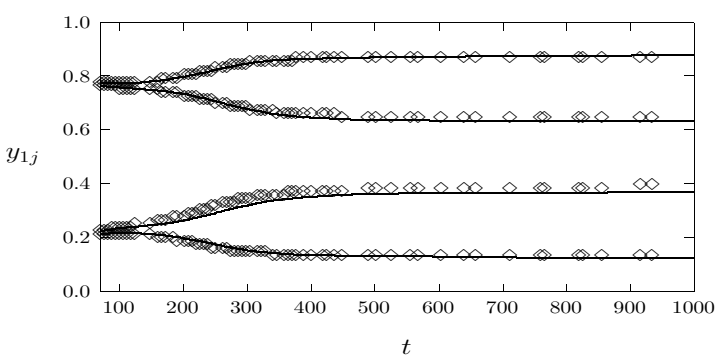

(b) $y_{j}$ vs. $t$

FiguRE 12. Comparison of the full numerical results computed numerically from (1.2) (discrete points) for the two cartesian coordinates of the spot locations in Fig. 1 with corresponding results (solid curves) computed from the asymptotic DAE system (3.6) and (3.17). The parameter values are as in Fig. 1 . The calibration is done at $t=70$. We only plot the trajectories of the four spots that have $x_{j}$ values closest to the center of the square.

\subsection{A One-Ring Pattern in the Unit Disk}

In this and the next subsection we consider two types of quasi-equilibrium spot patterns in the unit disk where the DAE system (3.6) and (3.17) can be studied analytically.

We first consider a quasi-equilibrium spot pattern in the unit disk where $K$ spots are equi-distributed on a ring of radius $r$ with $0<r<1$ at locations

$$
x_{j}=r e^{2 \pi i j / K}, \quad j=1, \ldots, K, \quad(\text { Pattern I }),
$$

where $i \equiv \sqrt{-1}$. For such a pattern the Green's matrix $\mathcal{G}$ is a symmetric circulant matrix with eigenvector $e=$ $(1, \ldots, 1)^{t}$ (see section 4 of $[\mathbf{2 6}]$ ). From Principal Result 3.3 above, the corresponding eigenvalue of $\mathcal{G}$ is $p_{K} / K$, where $p_{K} \equiv p_{K}(r)$ is given explicitly by (see Proposition 4.3 of $[\mathbf{2 6}]$ ),

$$
p_{K}(r) \equiv \sum_{j=1}^{K} \sum_{k=1}^{K} \mathcal{G}_{j, k}=\frac{1}{2 \pi}\left[-K \log \left(K r^{K-1}\right)-K \log \left(1-r^{2 K}\right)+r^{2} K^{2}-\frac{3 K^{2}}{4}\right] .
$$

The common spot strength is $S_{j}=S_{c}$, for $j=1, \ldots, K$, where $S_{c}$ is given in (3.12). For this type of ring pattern, the ODE system (3.17) can be readily written in gradient form as

$$
x_{j}^{\prime} \sim-\pi \varepsilon^{2} \gamma\left(S_{c}\right) S_{c} \nabla_{x_{j}} \mathcal{F}, \quad j=1, \ldots, K,
$$

where the function $\mathcal{F}\left(x_{1}, \ldots, x_{K}\right)$ is defined explicitly by

$$
\mathcal{F}\left(x_{1}, \ldots, x_{K}\right)=\sum_{i=1}^{K} R\left(x_{i} ; x_{i}\right)++\sum_{\substack{i=1 \\ i \neq j}}^{K} \sum_{j=1}^{K} G\left(x_{i} ; x_{j}\right) .
$$

In obtaining (5.5) from (5.6) and (3.17) we used the symmetry relations $G(x ; \xi)=G(\xi ; x)$ and $R(x ; \xi)=R(\xi ; x)$. 


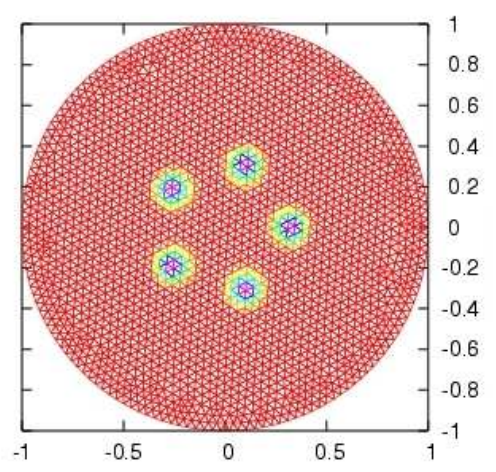

(a) $t=8.0$

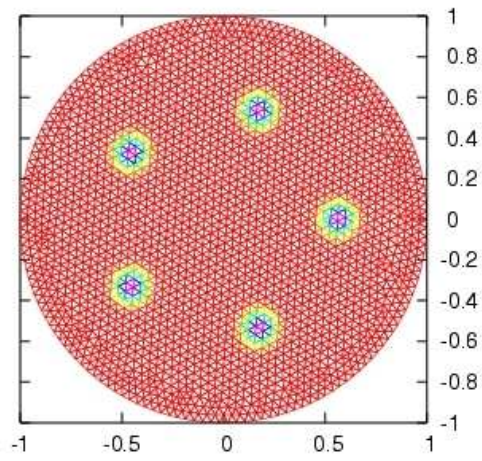

(b) $t=90.0$

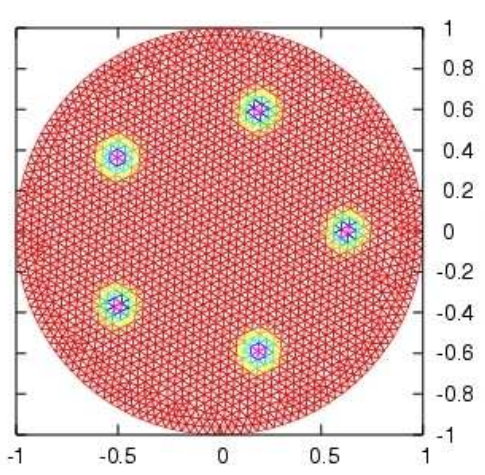

(c) $t=297.0$

FIGURE 13. Contour plot of $v$ computed numerically from (1.2) for an initial five-spot pattern on a ring inside the unit disk. The parameter values are $\varepsilon=0.02, a=35$, and $D=1$, and the initial ring radius is $r_{0}=0.3$. The spots remain on a ring of slowly increasing radius.

Finally, we use (5.6) and $x_{j}=r e^{2 \pi i j / K}$ to calculate

$$
\nabla_{x_{j}} \mathcal{F}\left(x_{1}, \ldots, x_{K}\right)=\frac{1}{K} p_{K}^{\prime}(r) e^{2 \pi i j / K}, \quad j=1, \ldots, K .
$$

Upon substituting (5.7) into (5.5), we obtain that the ring radius $r$ evolves slowly in time as

$$
r^{\prime}=-\left(\frac{\pi \varepsilon^{2}}{K}\right) \gamma\left(S_{c}\right) S_{c} p_{K}^{\prime}(r)
$$

where $p_{K}(r)$ is given in (5.4). Upon differentiating (5.4) with respect to $r$ we obtain the following main result:

Principal Result 5.1: Let $\Omega$ be the unit disk with center at $x=0$. At $t=0$ we assume that there are $K$ spots equi-distributed on a ring of radius $r_{0}$, with $0<r_{0}<1$, at the locations $x_{j}=r_{0} e^{2 \pi i j / K}$ for $j=1, \ldots, K$. Suppose that the common source strength $S_{c}=S_{j}$, for $j=1, \ldots, K$, as given in (3.12) satisfies $S_{c}<\Sigma_{2} \approx 4.3$. Then, under the DAE system (3.6) and (3.17), the $K$ spots remain equi-distributed on a ring of radius $r(t)$ for all time, where the slowly evolving ring radius satisfies the nonlinear first-order ODE

$$
r^{\prime}=-\varepsilon^{2} \gamma\left(S_{c}\right) S_{c}\left[-\frac{(K-1)}{2 r}+\frac{K r^{2 K-1}}{1-r^{2 K}}+r K\right],
$$

with $r(0)=r_{0}$. As $t \rightarrow \infty$, the ring radius approaches the unique minimum point $r_{e}$ of $p_{K}(r)$ given by the unique root in $0<r_{e}<1$ of the transcendental equation

$$
\frac{(K-1)}{2 K}-r^{2}=\frac{r^{2 K}}{1-r^{2 K}} .
$$

The roots of (5.10) are given explicitly in the second column of Table 1 of [26]. In particular, $r_{e}=0.5516$ when $K=3, r_{e}=0.6252$ when $K=5$, and $r_{e}=0.6604$ when $K=8$..

Experiment 6 (Equi-Distributed Spots on One Ring): For $\varepsilon=0.02, a=35$, and $D=1$, in Fig. 13 we show full numerical results computed from (1.2) for a five-ring pattern where the spots were initially equi-distributed on a ring of initial radius $r_{0}=0.3$. Since $S_{j}=3.5<\Sigma_{2}$ for $j=1, \ldots, 5$ from (3.6), the spots indeed remain equi-distributed on a slowly expanding ring for all time. In Fig. 14(a) we show a very favorable comparison between the asymptotic ring radius $r=r(t)$ as predicted by (5.9) and the results obtained from the full numerical solution of (1.2). In this figure, we also show a similar very favorable comparison between the asymptotic and full numerical results for either 


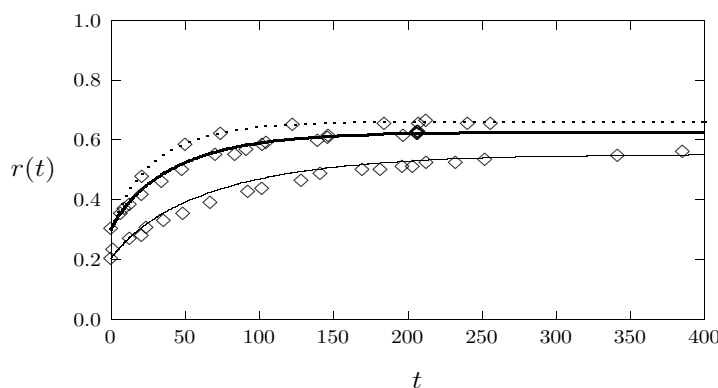

(a) Eight, Five, and Three Spots on a Ring

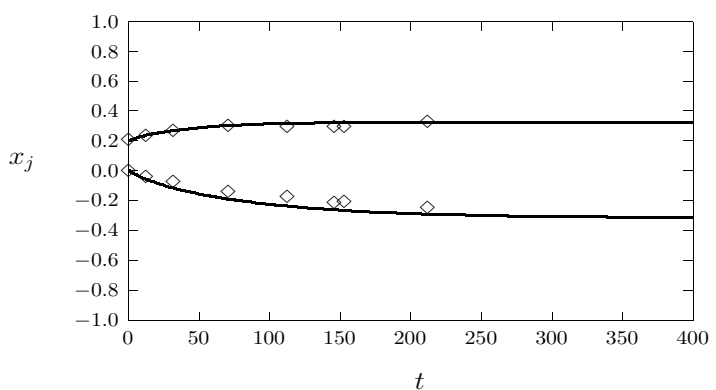

(b) Two Initial Non-Equi-Distributed Spots on a Ring

FiguRE 14. Left figure: the asymptotic ring radius vs. time from (5.9) for either eight spots (dashed line), five spots (heavy solid curve), or three spots (solid curve) equi-distributed on some initial ring inside the unit disk. The parameter values and initial ring radii are; $\varepsilon=0.02, a=54, D=1, r_{0}=0.3$, for the eight-spot pattern; $\varepsilon=0.02, a=35, D=1, r_{0}=0.3$, for the five-spot pattern; and $\varepsilon=0.02, a=20, D=1, r_{0}=0.2$, for the three-spot pattern. The discrete points are obtained from full numerical simulations of (1.2). Right figure: the $x$ cartesian coordinate of the two spot locations vs. $t$ for a two-spot pattern with initial spot locations $(0.2,0.0)$ and $(0.0,0.2)$, which are not initially equi-distributed on a ring. The spots remain on a slowly expanding ring for all time, and eventually become equi-distributed on the ring.

an eight-spot or a three-spot ring pattern. The parameter values chosen for these other examples are given in the caption of Fig. 14(a) and are such that the spot strengths $S_{j}$ are initially below the splitting threshold.

Experiment 7 (Non-Equi-Distributed Spots on One Ring): In Fig. 14(b) we plot the $x$-cartesian component of the spot locations for an initial two-spot pattern where the two spots are not equi-distributed on a ring. The parameter values are $\varepsilon=0.02, a=15, D=1$, and the initial spot locations are $x_{1}=(0.2,0.0)$ and $x_{2}=(0.0,0.2)$. For this parameter set $S_{j}=3.75<\Sigma_{2}$ at $t=0$ and the DAE system (3.6) and (3.17) predicts that $S_{j}$ remains below the spot-splitting threshold $\Sigma_{2}$ for all time. As $t$ increases, the two spots become equi-distributed on a slowly expanding ring. The ring radius approaches $r_{e} \approx 0.454$ as $t$ increases, which is consistent with (5.10).

Experiment 8 (Weak Instabilities for Spots on One-Ring): Next we show weak instabilities for an equidistributed one-ring pattern of spots when the number of spots on one ring exceeds some threshold. We first let nine spots be equi-distributed at $t=0$ on a ring of initial radius $r_{0}=0.3$ for the parameter values $\varepsilon=0.02, a=60$, and $D=1$. A small initial perturbation of the spot locations on the ring is then given to break the symmetry. The source strengths $S_{j}$ for $j=1, \ldots, 9$, as computed from the DAE system (3.6) and (3.17), are initially below and remain below the spot-splitting threshold $\Sigma_{2}$. In Fig. 15(a) we plot the distance to the origin as a function of time for each of the nine spots as computed from the DAE system. Initially the spots remain very close to a slowly expanding ring. However, as the nine-spot equilibrium radius $r_{e} \approx 0.666$ is approached, a weak instability in the DAE system is triggered and one of the spots is expelled from the ring. This spot then slowly drifts back to the center of the disk. The resulting equilibrium ring pattern has eight equi-distributed spots on a ring of radius $r_{e} \approx 0.709$ together with a spot at the center of the unit disk. This second type of ring pattern is constructed analytically in $\S 5.2$.

As a further illustration of this weak instability, we consider ten initial equi-distributed spots on a (slightly perturbed) ring of initial radius $r_{0}=0.3$ for the parameter values $\varepsilon=0.02, a=66$, and $D=1$. In Fig. 15 (b) we plot the distance to the origin as a function of time for each of the ten spots as computed from the DAE system (3.6) and (3.17). Although the ten spots remain very close to a slowly expanding ring for a long time, a weak instability 
is eventually triggered that eventually leads to an equi-distributed three-ring pattern with four spots on each of the two larger rings and two spots on the smaller ring.

It is beyond the scope of this paper to give a detailed analysis of this type of weak instability associated with equilibria of the DAE system (3.6) and (3.17). For a related, but considerably simpler, interacting particle system a similar type of small eigenvalue instability was studied analytically in [21].

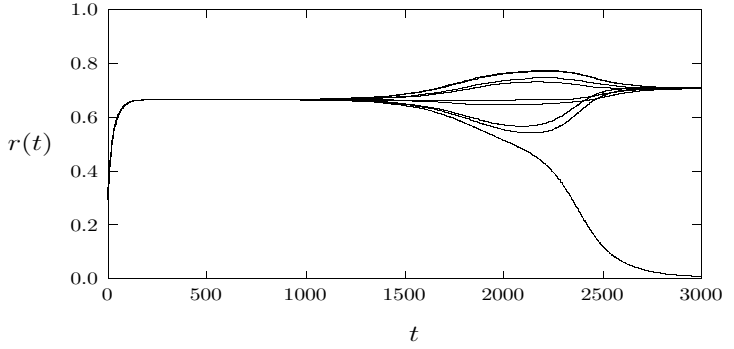

(a) $r_{j}$ vs. $t$

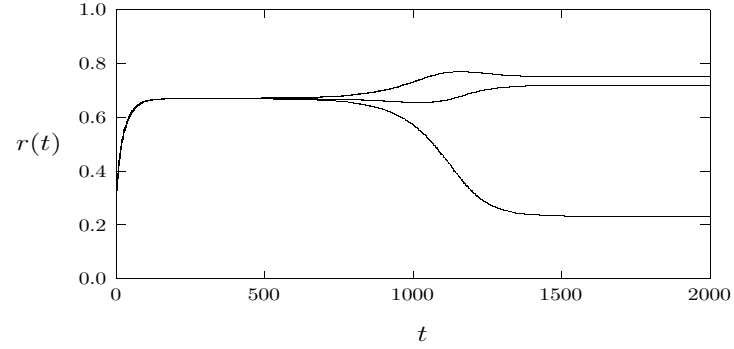

(b) $r_{j}$ vs. $t$

Figure 15. Asymptotic results for the unit disk, computed from the DAE system (3.6) and (3.17), for the distance of each spot to the center of the disk vs. time. Left figure: for $\varepsilon=0.02, a=60$, and $D=1$, the initial pattern has nine equi-distributed spots on a slightly perturbed ring of initial radius $r_{0}=0.3$. Initially the nine spots remain on a slowly expanding ring. However, the final equilibrium state has eight spots on a ring with a spot at the center of the disk. Right figure: for $\varepsilon=0.02, a=66$, and $D=1$, the initial pattern has ten equi-distributed spots on a slightly perturbed ring of initial radius $r_{0}=0.3$. The final equilibrium state is an equi-distributed three-ring pattern with four spots on each of the two larger rings and two spots on the smaller ring.

Experiment 9 (Spot-Splitting on One Ring): To illustrate spot-splitting behavior on a ring, we consider three spots that are initially equi-distributed on a ring of radius $r_{0}=0.3$ for the parameter values $\varepsilon=0.02, a=30$ and $D=1$. For this initial pattern, $S_{j}=5.0>\Sigma_{2}$ for $j=1, \ldots, 3$ from (3.6). Therefore, we predict that all of the spots will split simultaneously starting at $t=0$. This splitting process is shown in Fig. 16 where we plot full numerical solutions computed from (1.2). The spots are shown to split in a direction roughly tangential to the ring. The spots then become equi-distributed on a slowly expanding ring. The final six-spot pattern has a ring radius of $r \approx 0.642$, which is consistent with the equilibrium ring radius $r_{e}$ as predicted by (5.10).

\subsection{A One-Ring and Center Spot Pattern in the Unit Disk}

Next, we consider a quasi-equilibrium ring pattern in the unit disk with $K-1$ spots equi-distributed on a ring of radius $r$ and with an additional spot at the center of the disk. The points are labeled in complex form as

$$
x_{j}=r e^{2 \pi i j /(K-1)}, \quad j=1, \ldots, K-1, \quad x_{K}=0, \quad(\text { Pattern II }),
$$

where $i \equiv \sqrt{-1}$. The spots on the ring have a common source strength $S_{j}=S_{c}$ for $j=1, \ldots, K-1$, while the spot at the center has strength $S_{K}$.

We will assume that $K \geq 3$ so that there is at least two equi-distributed spots on the ring. For this case, (3.17) predicts that the center spot is stationary (i.e. $x_{K}^{\prime}=0$ ), while the $K-1$ spots on the ring satisfy

$$
x_{j}^{\prime}=-2 \pi \varepsilon^{2} \gamma\left(S_{c}\right)\left[\left(\frac{S_{c}}{2}\right) \nabla_{x_{j}} \mathcal{F}\left(x_{1}, \ldots, x_{K-1}\right)+S_{K} \nabla G\left(x_{j} ; 0\right)\right], \quad j=1 \ldots, K-1 .
$$




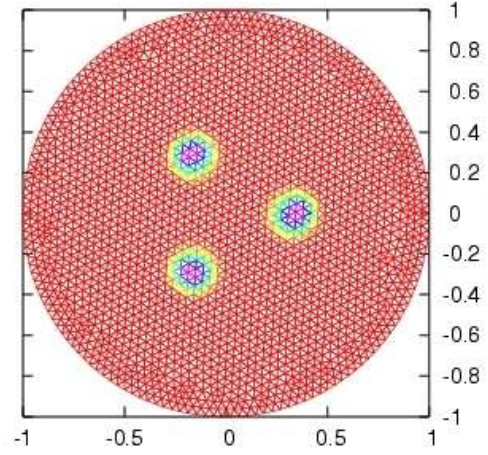

(a) $t=8.0$

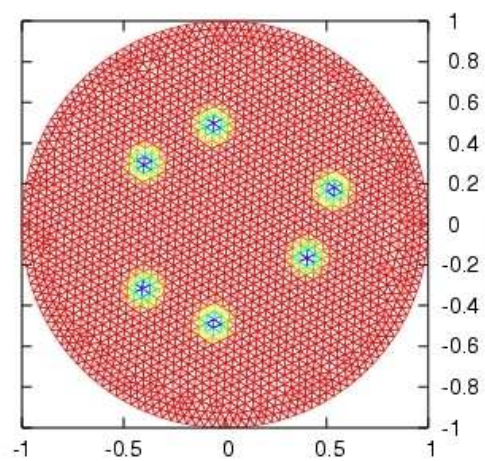

(d) $t=75.0$

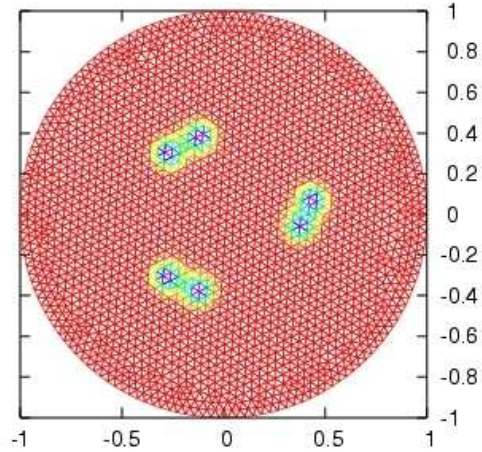

(b) $t=30.0$

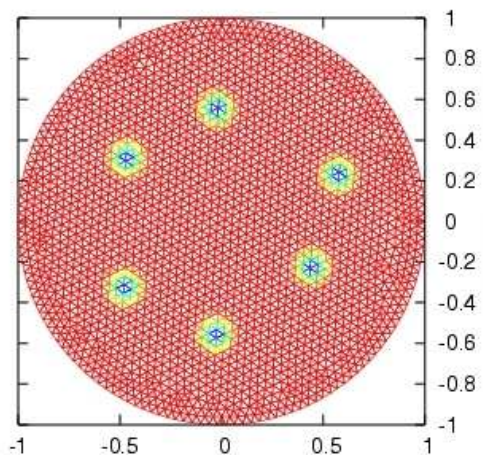

(e) $t=135.0$

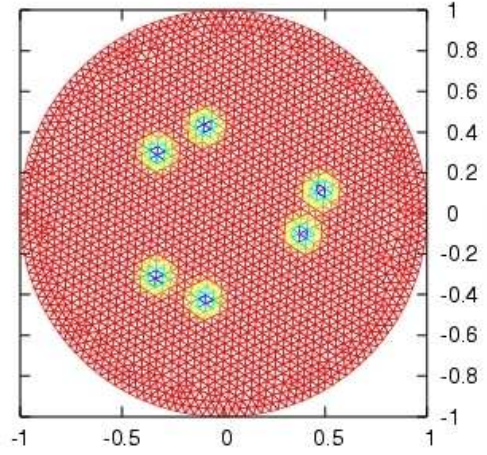

(c) $t=45.0$

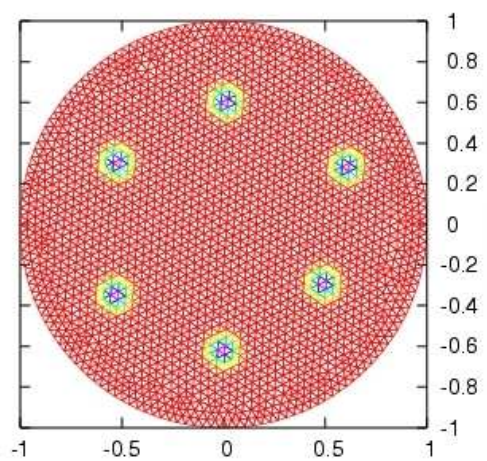

(f) $t=297.0$

Figure 16. Contour plot of $v$ computed from (1.2) for an initial three-spot pattern with spots equi-distributed on a ring of initial radius $r_{0}=0.3$. The parameter values are $\varepsilon=0.02, a=30$, and $D=1$. Each of the spots split leading to a six-spot pattern on a ring. The ring radius then slowly relaxes to its equilibrium value of $r_{e} \approx 0.642$ consistent with (5.10).

Here $\mathcal{F}\left(x_{1}, \ldots, x_{K}\right)$ was defined in (5.6). From (5.7) and (4.2) we calculate

$$
\nabla_{x_{j}} \mathcal{F}\left(x_{1}, \ldots, x_{K}\right)=\frac{1}{K-1} p_{K-1}^{\prime}(r) e^{2 \pi i j /(K-1)}, \quad j=1, \ldots, K-1, \quad \nabla G\left(x_{j} ; 0\right)=\frac{1}{2 \pi}\left(r-\frac{1}{r}\right) e^{2 \pi i j /(K-1)} .
$$

Upon substituting (5.13) into (5.12) we obtain an explicit ODE for the ring radius $r(t)$ in terms of the as yet unknown source strengths $S_{c}$ and $S_{K}$. The result for the asymptotic dynamics is given below in (5.17) of Principal Result 5.2.

The unknown source strengths $S_{c}$ and $S_{K}$ can be obtained from (3.7). Upon defining $S_{v}=\left(S_{c}, \ldots, S_{c}, S_{K}\right)^{t}$, and by using $(5.11)$ for $x_{j}$, we readily calculate the $j^{\text {th }}$ row, $\left(\mathcal{G} S_{v}\right)_{j}$, of the matrix vector product $\mathcal{G} S_{v}$ in $(3.7)$ as

$$
\left(\mathcal{G} S_{v}\right)_{j}=\frac{p_{K-1} S_{c}}{K-1}+\alpha S_{K}, \quad j=1, \ldots, K-1 ; \quad\left(\mathcal{G} S_{v}\right)_{K}=(K-1) \alpha S_{c}+\beta S_{K} .
$$

Here $p_{K-1}=p_{K-1}(r)$ is defined in (5.4), while $\alpha=\alpha(r)$ and $\beta=\beta(r)$ are defined in terms of the Green's function and its regular part by

$$
\alpha \equiv G(r ; 0)=-\frac{1}{2 \pi} \log r+\frac{r^{2}}{4 \pi}-\frac{3}{8 \pi}, \quad \beta \equiv R(0 ; 0)=-\frac{3}{8 \pi} .
$$


Then, by substituting (5.14) into (3.7), we readily derive the following two equations for $S_{c}$ and $S_{K}$ :

$$
\begin{gathered}
S_{c}-S_{K}+2 \pi \nu\left[\frac{p_{K-1} S_{c}}{K-1}-(K-1) \alpha S_{c}+(\alpha-\beta) S_{K}\right]+\nu\left[\chi\left(S_{c}\right)-\chi\left(S_{K}\right)\right]=0, \\
(K-1) S_{c}+S_{K}=\mu, \quad \mu \equiv \frac{a|\Omega|}{2 \pi \sqrt{D}} .
\end{gathered}
$$

By eliminating $S_{K}$ in (5.16) we obtain a nonlinear algebraic equation for $S_{c}$. The result is summarized as follows:

Principal Result 5.2: Let $\Omega$ be the unit disk with center at $x=0$. At $t=0$ we assume that $K-1$ spots, with $K \geq 3$, are equi-distributed on a ring of radius $r_{0}$, with $0<r_{0}<1$, at the locations $x_{j}=r_{0} e^{2 \pi i j /(K-1)}$ for $j=1, \ldots, K-1$, and that there is a spot at the center $x_{K}=0$ of the disk. Then, assuming that $S_{c}<\Sigma_{2} \approx 4.3$ and $S_{K}<\Sigma_{2}$ for each $t \geq 0$, as $t$ increases the center spot $x_{K}$ remains at the origin while the other $K-1$ spots remain equi-distributed on a ring of radius $r(t)$, where $r(t)$ with $r(0)=r_{0}$ satisfies the nonlinear first-order $O D E$

$$
r^{\prime}=-\varepsilon^{2} \gamma\left(S_{c}\right) S_{c}\left[-\frac{(K-2)}{2 r}+\frac{(K-1) r^{2 K-3}}{1-r^{2 K-2}}+r(K-1)+\frac{S_{K}}{S_{c}}\left(r-\frac{1}{r}\right)\right] .
$$

The equilibrium ring radius $r_{e}$ of (5.17), with $0<r_{e}<1$, is given by the root(s) of

$$
\frac{\left(S_{K} / S_{c}+(K-2) / 2\right)}{(K-1)+S_{K} / S_{c}}-r^{2}=\left(\frac{(K-1)}{(K-1)+S_{K} / S_{c}}\right) \frac{r^{2 K-2}}{1-r^{2 K-2}} .
$$

In (5.17), $S_{c}(r)$ is the common source strength for the spots on the ring (i.e. $S_{j}=S_{c}$ for $j=1, \ldots, K-1$ ), which is a root of the nonlinear algebraic equation

$$
S_{c}\left[1+\frac{2 \pi \nu}{K}\left(\frac{p_{K-1}}{K-1}+(K-1)(\beta-2 \alpha)\right)\right]+\frac{\mu}{K}[2 \pi \nu(\alpha-\beta)-1]=\frac{\nu}{K}\left[\chi\left(\mu-S_{c}(K-1)\right)-\chi\left(S_{c}\right)\right],
$$

on the interval $0<S_{c}<\mu /(K-1)$. In terms of $S_{c}$, the source strength $S_{K}=S_{K}(r)$ of the center spot is given by $S_{K}=\mu-(K-1) S_{c}$. Here $\nu=-1 / \log \varepsilon, \alpha=\alpha(r)$ and $\beta=\beta(r)$ are defined in (5.15), and $\mu$ is defined in (5.16b).

Principal Result 5.2 shows that the existence and dynamics of the quasi-equilibrium $K$-spot ring pattern (5.11) is reduced to the study of the coupled DAE system for the dynamics of the ring radius (5.17) and the single nonlinear algebraic equation (5.19). In Fig. 17 we plot the numerical solution to (5.19) for $S_{c}=S_{c}(r)$ and $S_{K}=S_{K}(r)$ as a function of $r$ for $\varepsilon=0.02, D=1$, but for several different values of $a$ and $K$. The results show that the common ring spot strength $S_{c}$ has a fold point behavior with respect to $r$. Hence, quasi-equilibrium ring patterns of the type (5.11) exhibit bistable phenomena and exist only when $r$ exceeds some fold point value $r_{f}$. For $r>r_{f}$, there are exactly two values of $S_{c}$ with $S_{c}=\mathcal{O}(1)$. Since $S_{K}=\mu-(K-1) S_{c}$, the upper (lower) branches for $S_{c}$ vs. $r$ in Fig. 17(a) and Fig. 17(c) correspond to the lower (upper) branches for $S_{K}$ vs. $r$ in Fig. 17(b) and Fig. 17(d), respectively. In Fig. 17 the equilibrium ring radius $r_{e}$ for the dynamics (5.17) is indicated. The crosses in Fig. 17 indicate those points where either $S_{c}$ or $S_{K}$ is at the spot-splitting threshold $\Sigma_{2} \approx 4.3$.

In Fig. 18(a) we plot the numerical solution $r$ vs. $t$ to the reduced DAE system (5.17) and (5.19) with initial value $r(0)=0.55$ corresponding to the lower branches of the solid curves for $S_{c}$ vs. $r$ in Fig. 17(a) and Fig. 17(c). The solid curve in Fig. 18(a) for the parameter set $\varepsilon=0.02, a=60, D=1$, and $K=9$, has an equilibrium ring radius of $r_{e} \approx 0.709$. This parameter set and equilibrium ring radius is precisely the value obtained from the full numerical solutions of (1.2) shown in Fig. 15(a) of Experiment 8 in $\S 5.1$.

Experiment 10 (Convergence to a Ring with Center Spot Pattern): Ring patterns of the type (5.11) can also arise as the steady-state limit of an arbitrary arrangement of initial spots locations in the unit disk. An example of this is shown in Fig. 19 for the parameter set $\varepsilon=0.02, a=36, D=1$, and $K=6$, with initial spot locations as 


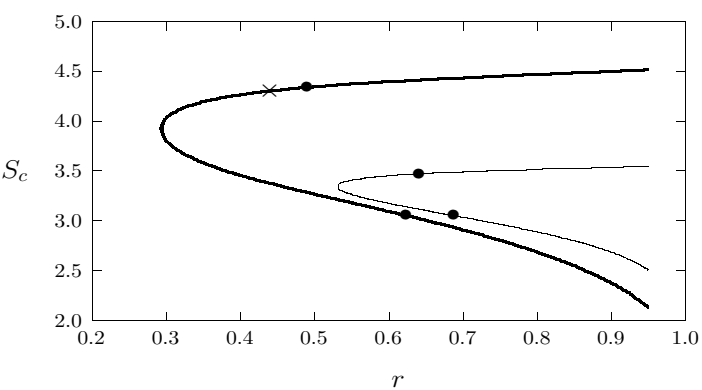

(a) $S_{c}$ vs. $r$

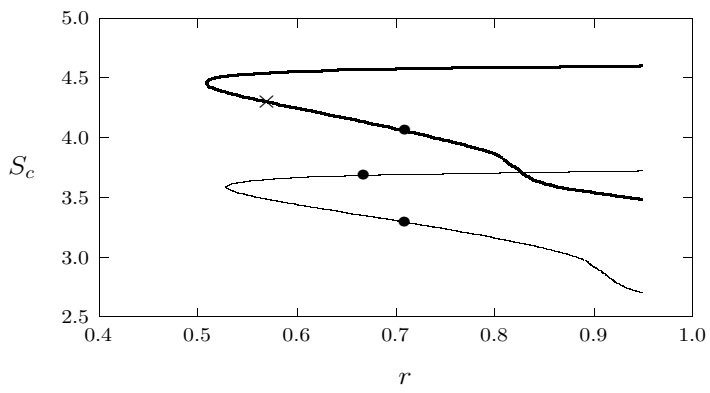

(c) $S_{c}$ vs. $r$

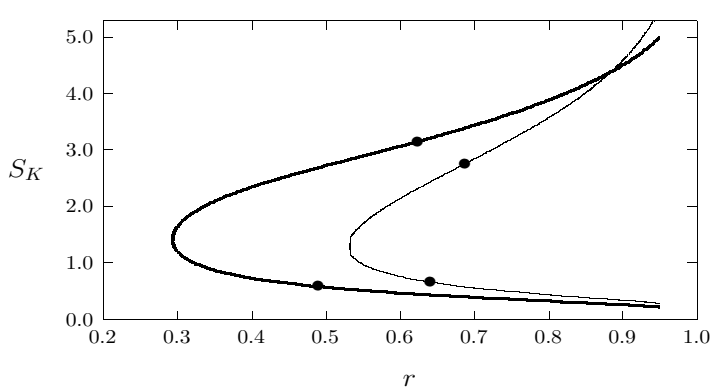

(b) $S_{K}$ vs. $r$

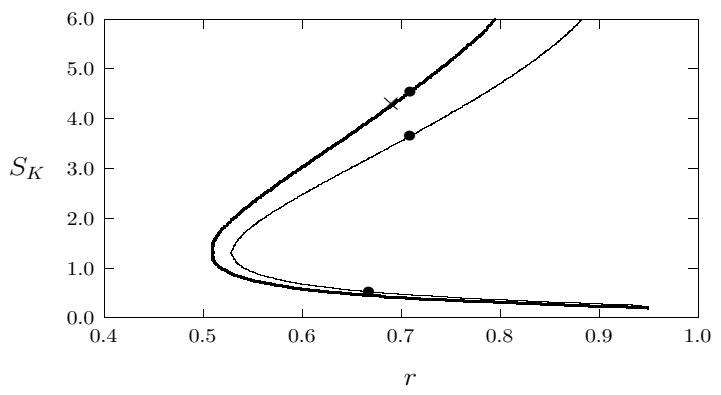

(d) $S_{K}$ vs. $r$

Figure 17. $S_{c}$ vs. $r$ (left figures) and $S_{K}$ vs. $r$ (right figures) for the ring pattern (5.11) in the unit disk calculated from (5.19) for $\varepsilon=0.02$ and $D=1$. Top row: $K=3$, and $a=18.5$ (heavy solid curves); $K=6$, and $a=36$ (solid curves). Bottom Row: $K=9$, and $a=74$ (heavy solid curves); $K=9$ and $a=60$ (solid curves). The bullets are the equilibrium points for the dynamics (5.17). The crosses indicate where either $S_{c}$ or $S_{K}$ is at the spot-splitting threshold $\Sigma_{2}$.

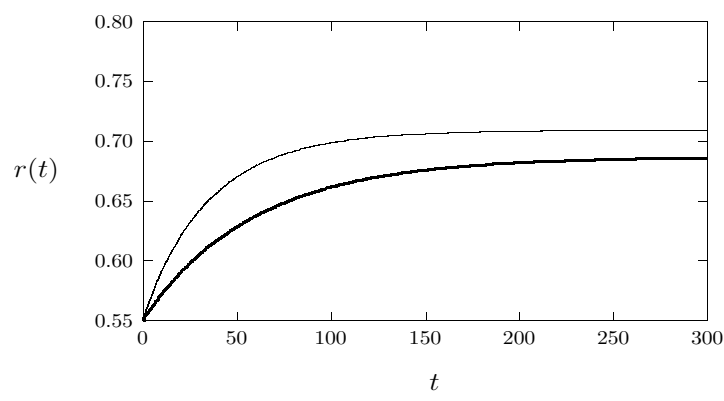

(a) $r$ vs. $t$

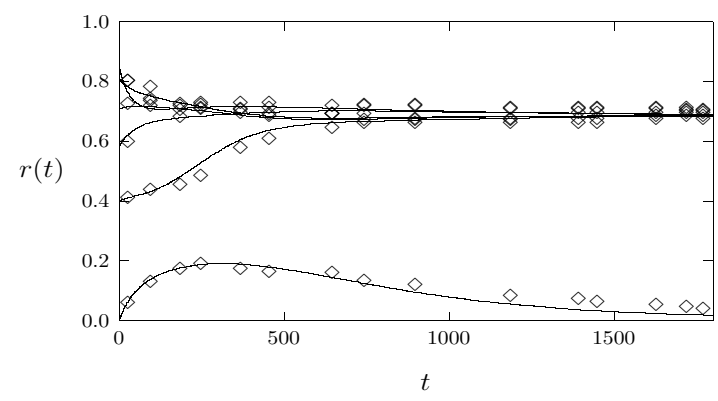

(b) $r_{j}$ vs. $t$

Figure 18. Left figure: asymptotic ring radius vs. $t$ with $r(0)=0.55, \varepsilon=0.02$ and $D=1$ for the ring pattern (5.11) in the unit disk computed from (5.17) and (5.19) for two lower solution branches in Fig. 17(a) and Fig. 17 (c); $a=36$ and $K=6$ (heavy solid curve); $a=60$ and $K=9$ (solid curve). Right figure: distances $r_{j}$ vs. $t$ for each of the six spots shown in Fig. 19 . The solid curves are obtained by solving the DAE system (3.6) and (3.17) numerically, while the discrete points are computed numerically from (1.2). As $t$ increases the ring radius approaches $r_{e} \approx 0.68$, which is consistent with the equilibrium point for the heavy solid curve in the left figure. 


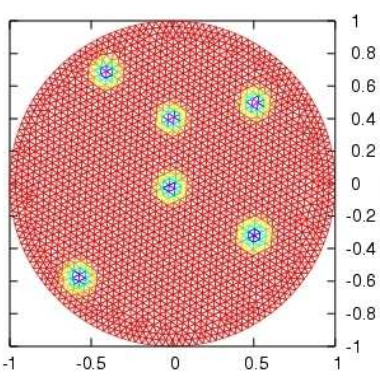

(a) $t=23.0$

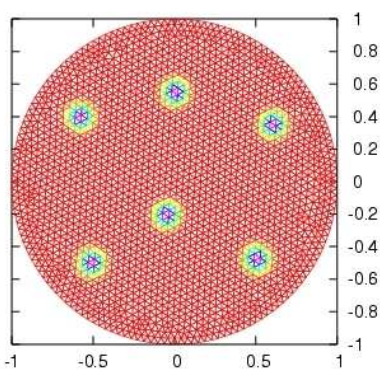

(b) $t=418.0$

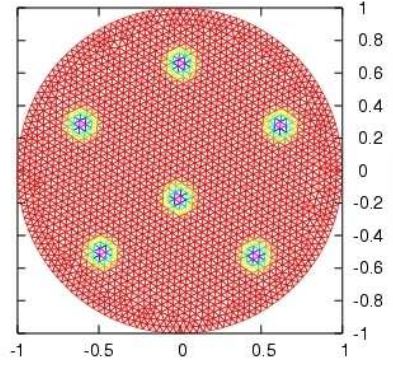

(c) $t=922.0$

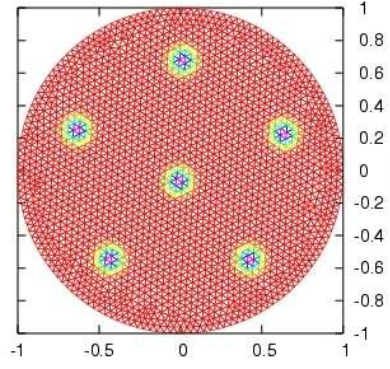

(d) $t=1880.0$

FiguRE 19. Numerical results computed from (1.2) in the unit disk for an initial six spot pattern with spot locations $x_{1}=(0,0)$, $x_{2}=(0.0,0.4), x_{3}=(-0.6,-0.6), x_{4}=(0.5,-0.3), x_{5}=(0.5,0.5)$, and $x_{6}=(-0.4,0.7)$. The parameter values are $\varepsilon=0.02$, $a=36$, and $D=1$. The final equilibrium state has five spots on a ring together with a spot at the center of the disk.

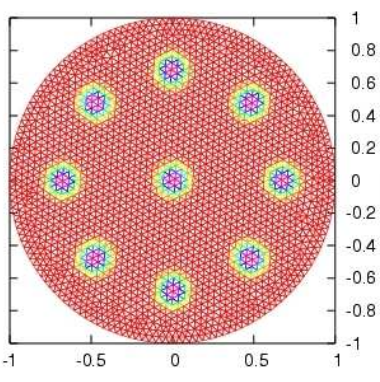

(a) $t=54.0$

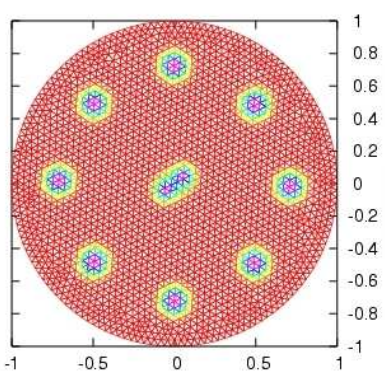

(b) $t=372.0$

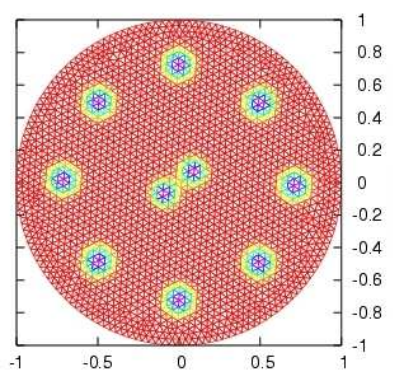

(c) $t=387.0$

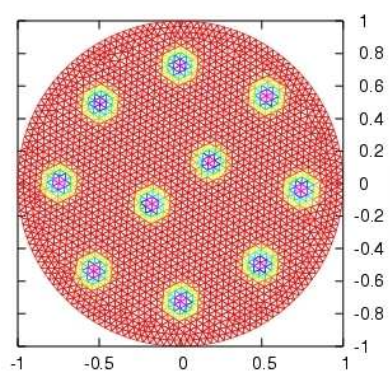

(d) $t=800.0$

FIGURE 20. Numerical results computed from (1.2) in the unit disk for an initial pattern with eight spots equi-distributed on a ring of initial radius $r_{0}=0.63$ together with a spot at the center of the disk. The parameter values are $\varepsilon=0.02, a=74$, and $D=1$. The ring slowly expands and the spot at the center undergoes a splitting process resulting in an equilibrium solution that has two equi-distributed spots on an inner ring and eight such spots on an outer ring.

given in the caption of Fig. 19. In Fig. 18(b) we compare the asymptotic results from the full DAE system (3.6) and (3.17) with corresponding numerical results computed from (1.2) for the distance of each of the spots to the center of the unit disk. The final equilibrium pattern has five spots equi-distributed on a ring with a spot at the center of the unit disk. The equilibrium ring radius $r_{e} \approx 0.68$ for this pattern is precisely the equilibrium value for the lower branch on the solid curve in Fig. 17(a). Therefore, as $t \rightarrow \infty$, the initial pattern in Fig. 19 approaches the equilibrium point on the lower branch of the solid curve in Fig. 17(a).

Finally, we use the plots of $S_{c}$ and $S_{K}$ versus $r$ in Fig. 17 to show that a ring pattern of the form (5.11) that is initially stable to spot-splitting at $t=0$ can become unstable to spot-splitting at a later time before the ring radius reaches its steady-state limiting value. This is referred to as a dynamically induced or triggered instability. Different types of dynamically induced instabilities are well-known to occur for spike solutions in one-spatial dimension (see [41]). To illustrate this behavior in our two-dimensional setting we now consider a specific example.

Experiment 11 (Dynamic Instability for a Ring with Center Spot Pattern): Consider the parameter set $\varepsilon=0.02, a=74, D=1$, and $K=9$, and let $r(0)=0.63$ be the initial ring radius. This gives an initial point on the lower branch of the $S_{c}$ versus $r$ (heavy solid) curve in Fig. 17(c) and, correspondingly, an initial point on 
the upper branch of the $S_{K}$ versus $r$ (heavy solid curve) in Fig. 17(d). At this initial ring radius $r(0)=0.63$, we calculate from (5.19) that $S_{c} \approx 4.19$ and $S_{K} \approx 3.45$, which are both below the spot-splitting threshold $\Sigma_{2} \approx 4.3$. As $t$ increases, the ring radius slowly expands and $S_{K}$ slowly increases on the upper branch of the heavy solid curve in Fig. 17(d). However, we calculate that $S_{K} \approx 4.54>\Sigma_{2}$ at the equilibrium value $r=r_{e} \approx 0.709$, and $S_{K} \approx 4.3$ when $r \approx 0.69$. Therefore, the source strength $S_{K}$ for the spot at the center exceeds the spot-splitting threshold $\Sigma_{2}$ before the equilibrium ring radius is attained. Theoretically, we then predict that the spot at the center will undergo a spot-splitting event through a dynamically triggered instability. In Fig. 20 we show a confirmation of this prediction from full numerical solution of (1.2).

A very similar argument shows that a dynamically triggered instability will occur for each spot on the ring for the parameter set $\varepsilon=0.02, a=18.5, D=1$, and $K=3$, with initial ring radius $r(0)=0.33$, corresponding to an initial point on the upper branch of the $S_{c}$ versus $r$ (heavy solid) curve in Fig. 17(a). At $t=0$ and $r(0)=0.33$, we compute from (5.19) that $S_{c} \approx 4.15$ and $S_{K} \approx 0.95$, and that $S_{c} \approx 4.35$ when $r=r_{e} \approx 0.489$. Therefore, $S_{c}$ exceeds the spot-splitting value for some $r$ in $r(0)<r<r_{e}$, leading to a dynamically triggered spot-splitting behavior for the two spots on the ring.

\section{The NLEP Regime: Stability Analysis for $D=\mathcal{O}\left(\nu^{-1}\right)$}

We now consider the limiting case $D \gg 1$ with $D=D_{0} / \nu$, where $\nu \equiv-1 / \log \varepsilon$ and $D_{0}$ is an $\mathcal{O}(1)$ parameter. In this regime, it was shown in [54] that the stability of a multi-spot pattern is determined by a certain nonlocal eigenvalue problem (NLEP). By applying stability results of [48] and [52] for this NLEP, it was proved in [54] that a $K$-spot pattern, with spots of equal amplitude, is stable when $K>1$ if and only if

$$
D_{0} \leq D_{0 K} \equiv \frac{a^{2}|\Omega|^{2}}{4 \pi^{2} K^{2} b_{0}}, \quad b_{0} \equiv \int_{0}^{\infty} \rho[w(\rho)]^{2} d \rho
$$

There is no stability threshold for a one-spot pattern when $D_{0}=\mathcal{O}(1)$. Here $w(\rho)$ is the unique positive radially symmetric ground-state solution of $\Delta y w-w+w^{2}=0$ in $\mathbb{R}^{2}$. In this section we show how to recover this result from the eigenvalue problem (3.26) and (3.20) of $\S 3$ in the regime $D=D_{0} / \nu$.

To do so, we first must construct approximate solutions to the core problems (3.2) and to the nonlinear system (3.6) for the source strengths when $D=D_{0} / \nu \gg 1$. The constraint in (3.6) indicates that $S_{j}=\mathcal{O}\left(\nu^{1 / 2}\right)$ when $D=\mathcal{O}\left(\nu^{-1}\right)$. This fact suggests that we must expand $S_{j}, U_{j}, V_{j}$, and $\chi_{j}$, in (3.2) as

$$
\left(\begin{array}{c}
S_{j} \\
V_{j}
\end{array}\right)=\nu^{1 / 2}\left[\left(\begin{array}{c}
S_{0 j} \\
V_{0 j}
\end{array}\right)+\nu\left(\begin{array}{c}
S_{1 j} \\
V_{1 j}
\end{array}\right)+\cdots\right], \quad\left(\begin{array}{c}
\chi_{j} \\
U_{j}
\end{array}\right)=\nu^{-1 / 2}\left[\left(\begin{array}{c}
\chi_{0 j} \\
U_{0 j}
\end{array}\right)+\nu\left(\begin{array}{c}
\chi_{1 j} \\
U_{1 j}
\end{array}\right)+\cdots\right] .
$$

Upon substituting (6.2) into (3.2), and collecting powers of $\nu$, we obtain that $U_{0 j}$ and $V_{0 j}$ satisfy

$$
\begin{gathered}
V_{0 j}^{\prime \prime}+\frac{1}{\rho} V_{0 j}^{\prime}-V_{0 j}+U_{0 j} V_{0 j}^{2}=0 ; \quad U_{0 j}^{\prime \prime}+\frac{1}{\rho} U_{0 j}^{\prime}=0, \quad 0 \leq \rho<\infty, \\
V_{0 j} \rightarrow 0, \quad U_{0 j} \rightarrow \chi_{0 j} \quad \text { as } \quad \rho \rightarrow \infty .
\end{gathered}
$$

At next order, $U_{1 j}$ and $V_{1 j}$ satisfy

$$
\begin{gathered}
V_{1 j}^{\prime \prime}+\frac{1}{\rho} V_{1 j}^{\prime}-V_{1 j}+2 U_{0 j} V_{0 j} V_{1 j}=-U_{1 j} V_{0 j}^{2} ; \quad U_{1 j}^{\prime \prime}+\frac{1}{\rho} U_{1 j}^{\prime}=U_{0 j} V_{0 j}^{2}, \quad 0 \leq \rho<\infty, \\
V_{1 j} \rightarrow 0, \quad U_{1 j} \rightarrow S_{0 j} \log \rho+\chi_{1 j} \quad \text { as } \quad \rho \rightarrow \infty .
\end{gathered}
$$


Then, at one higher order, we get that $U_{2 j}$ satisfies

$$
U_{2 j}^{\prime \prime}+\frac{1}{\rho} U_{2 j}^{\prime}=U_{1 j} V_{0 j}^{2}+2 U_{0 j} V_{0 j} V_{1 j}, \quad 0 \leq \rho<\infty ; \quad U_{2 j} \sim S_{1 j} \log \rho+\chi_{2 j} \quad \text { as } \quad \rho \rightarrow \infty .
$$

The solution to $(6.3 a)$ with far-field conditions $(6.3 b)$ is simply

$$
U_{0 j}=\chi_{0 j}, \quad V_{0 j}=w / \chi_{0 j} .
$$

Here $\chi_{0 j}$ is a constant to be found, and $w(\rho)$ is the radially symmetric ground-state solution of $\Delta_{y} w-w+w^{2}=0$. To determine $S_{0 j}$ we apply the Divergence theorem to the $U_{1 j}$ equation in $(6.3 c)$ to obtain

$$
S_{0 j}=\int_{0}^{\infty} \rho U_{0 j} V_{0 j}^{2} d \rho=\frac{b_{0}}{\chi_{0 j}}, \quad b_{0} \equiv \int_{0}^{\infty} \rho w^{2} d \rho
$$

It is then convenient to decompose $U_{1 j}$ and $V_{1 j}$ in terms of new variables $\hat{U}_{1}$ and $\hat{V}_{1}$ by

$$
U_{1 j}=\frac{1}{\chi_{0 j}}\left[\chi_{0 j} \chi_{1 j}+\hat{U}_{1}\right], \quad V_{1 j}=\frac{1}{\chi_{0 j}^{3}}\left[-\chi_{0 j} \chi_{1 j} w+\hat{V}_{1}\right] .
$$

Upon substituting (6.4), (6.5), and (6.6), into $(6.3 c)$ and $(6.3 d)$, and using the identity $\Delta_{y} w-w+2 w^{2}=w^{2}$, we readily obtain that $\hat{U}_{1}$ and $\hat{V}_{1}$ are radially symmetric solutions of

$$
\mathcal{L}_{0} \hat{V}_{1}=-\hat{U}_{1} w^{2}, \quad \hat{U}_{1}^{\prime \prime}+\frac{1}{\rho} \hat{U}_{1}^{\prime}=w^{2}, \quad 0 \leq \rho<\infty ; \quad \hat{V}_{1} \rightarrow 0, \quad \hat{U}_{1}-b_{0} \log \rho \rightarrow 0 \quad \text { as } \rho \rightarrow \infty,
$$

with $\hat{V}_{1}^{\prime}(0)=\hat{U}_{1}^{\prime}(0)=0$. Here we have defined the "local" operator $\mathcal{L}$ in terms of $w$ by

$$
\mathcal{L} \Phi \equiv \Phi^{\prime \prime}+\frac{1}{\rho} \Phi^{\prime}-\Phi+2 w \Phi
$$

By specifying that there is no $\mathcal{O}(1)$ term in the far-field asymptotics of $\hat{U}_{1}$ in (6.7), then $\hat{U}_{1}$ and $\hat{V}_{1}$ are independent of $j$ and can be uniquely determined. Finally, we use the Divergence theorem on the $U_{2 j}$ equation in (6.3e) to determine $S_{1 j}$, and we simplify the resulting expression using (6.4) and (6.6). In this way, we obtain for $S_{1 j}$ that

$$
S_{1 j}=-\chi_{1 j}\left(\frac{b_{0}}{\chi_{0 j}^{2}}\right)+\frac{b_{1}}{\chi_{0 j}^{3}}, \quad b_{1} \equiv \int_{0}^{\infty} \rho\left(\hat{U}_{1} w^{2}+2 w \hat{V}_{1}\right) d \rho .
$$

Equations (6.5) and (6.9) relate $S_{0 j}$ and $S_{1 j}$ to the as yet unknown constants $\chi_{0 j}$ and $\chi_{1 j}$. To obtain a closed system of equations, we substitute (6.2) into (3.6) and set $D=D_{0} / \nu$. Then, we expand $u_{c}$ in (3.6) as

$$
u_{c}=\nu^{-1 / 2}\left(u_{c 0}+\nu u_{c 1}+\cdots\right) .
$$

Upon collecting powers of $\nu$ in the resulting expressions, we obtain that

$$
\begin{array}{cl}
S_{0 j}+\chi_{0 j}=-2 \pi u_{c 0}, \quad j=1, \ldots, K ; \quad & \sum_{j=1}^{K} S_{0 j}=\frac{a|\Omega|}{2 \pi \sqrt{D_{0}}}, \\
S_{1 j}+\chi_{1 j}+2 \pi R_{j, j} S_{0 j}+2 \pi \sum_{\substack{i=1 \\
i \neq j}}^{K} G_{i, j} S_{0 i}=-2 \pi u_{c 1}, \quad j=1, \ldots, K ; \quad \sum_{j=1}^{K} S_{1 j}=0 .
\end{array}
$$

We look for a $K$-spot solution with spots of equal height. Therefore, upon recalling (6.5) for $\chi_{0 j}$, we can solve (6.11a) to obtain $u_{c 0}$ and the common values for $S_{0 j}$ and $\chi_{0 j}$ given by

$$
S_{0 j}=S_{0} \equiv \frac{a|\Omega|}{2 \pi K \sqrt{D_{0}}}, \quad u_{c 0}=-\frac{1}{2 \pi}\left(S_{0}+\frac{b_{0}}{S_{0}}\right), \quad \chi_{0 j}=\chi_{0} \equiv \frac{b_{0}}{S_{0}} .
$$


To determine $S_{1 j}, \chi_{1 j}$, and $u_{c 1}$, we first substitute (6.9) for $S_{1 j}$ into $(6.11 b)$, and then solve the resulting system for $\chi_{1 j}$ and $u_{c 1}$. A simple calculation yields a linear system for $\chi_{v 1} \equiv\left(\chi_{11}, \ldots, \chi_{1 K}\right)^{t}$ in the form

$$
(I-\mu \mathcal{E}) \chi_{v 1}=-\mu\left[\frac{b_{1}}{\chi_{0}^{3}} e+2 \pi S_{0}\left(\mathcal{G}-\frac{p}{K} I\right) e\right] .
$$

In terms of this solution, $u_{c 1}$ is given by

$$
u_{c 1}=-\frac{1}{2 \pi K}\left(e^{t} \chi_{v 1}+2 \pi S_{0} p\right)
$$

In $(6.13), \mathcal{G}$ is the Green's function matrix of $(3.8), e \equiv(1, \ldots, 1)^{t}$ and

$$
\mathcal{E} \equiv \frac{1}{K} e e^{t}, \quad p=p\left(x_{1}, \ldots, x_{K}\right) \equiv e^{t} \mathcal{G} e, \quad \mu^{-1} \equiv 1-b_{0} / \chi_{0}^{2} .
$$

Since $\mathcal{E}$ has rank one, together with $\mathcal{E} e=e$ and $\mu \neq 1$, then $(I-\mu \mathcal{E})^{-1}$ exists and is readily calculated with the Sherman-Woodbury-Morrison formula. Finally, in terms of the solution to $(6.13 a), S_{1 j}$ is given by (6.9). We remark that when the spot locations $x_{1}, \ldots, x_{K}$ are such that $e$ is an eigenvector of $\mathcal{G}$ (i.e. $\mathcal{G}$ is a circulant matrix as in $\S 5.1$ ), then $\chi_{v 1}$ is given by the common value $\chi_{v 1}=\mu b_{1} e /\left[\chi_{0}^{3}(\mu-1)\right]$ from $(6.13 a)$. This completes the derivation of the approximate solution to (3.2) and (3.6) when $D=D_{0} / \nu \gg \mathcal{O}(1)$ with $D_{0}=\mathcal{O}(1)$.

Next, we show that for $D=D_{0} / \nu$ the multi-spot stability problem (3.26) and (3.20) reduces to the NLEP problem of [54]. Since $V_{j} \sim \nu^{1 / 2} w / \chi_{0 j}$ and $U_{j} \sim \nu^{-1 / 2} \chi_{0 j}$ from (6.2) and (6.4), then (3.20) with $D=D_{0} / \nu$ reduces to

$$
\begin{gathered}
\mathcal{L} \Phi_{j}+\frac{D_{0} w^{2}}{\chi_{0 j}} N_{j}=\lambda \Phi_{j}, \quad N_{j}^{\prime \prime}+\frac{1}{\rho} N_{j}^{\prime}=\nu\left(\frac{w^{2}}{\chi_{0 j}} N_{j}+\frac{2 w}{D_{0}} \Phi_{j}\right), \quad 0 \leq \rho<\infty, \\
\Phi_{j} \rightarrow 0, \quad N_{j} \sim C_{j} \log \rho+B_{j} \quad \text { as } \quad \rho \rightarrow \infty .
\end{gathered}
$$

Here $\mathcal{L} \Phi_{j} \equiv \Phi_{j}^{\prime \prime}+\rho^{-1} \Phi_{j}^{\prime}-\Phi_{j}+2 w \Phi_{j}$. Therefore, for $\nu \ll 1$, we conclude that $C_{j}=\mathcal{O}(\nu)$ and that $B_{j}$ is arbitrary. This motivates the expansion

$$
B_{j}=B_{j 0}+\nu B_{j 1}+\mathcal{O}\left(\nu^{2}\right), \quad C_{j}=\nu C_{j 0}+\mathcal{O}\left(\nu^{2}\right), \quad N_{j}=B_{j 0}+\nu N_{j 1}+\mathcal{O}\left(\nu^{2}\right) .
$$

Upon substituting (6.15) into (6.14), and collecting linear terms in $\nu$, we find that $N_{j 1}$ satisfies

$$
N_{j 1}^{\prime \prime}+\frac{1}{\rho} N_{j 1}^{\prime}=\left(\frac{w^{2}}{\chi_{0 j}} B_{j 0}+\frac{2 w}{D_{0}} \Phi_{j}\right), \quad 0<\rho<\infty ; \quad N_{j 1} \sim C_{j 0} \log \rho+B_{j 1} \quad \text { as } \quad \rho \rightarrow \infty .
$$

The Divergence theorem on the $N_{j 1}$ equation then determines $C_{j 0}$ in terms of $B_{j 0}$ as

$$
C_{j 0}=\frac{b_{0}}{\chi_{0 j}} B_{j 0}+\frac{2}{D_{0}} \int_{0}^{\infty} \rho w \Phi_{j} d \rho, \quad b_{0} \equiv \int_{0}^{\infty} \rho w^{2} d \rho .
$$

Next, we substitute (6.15) into (3.26) to obtain to leading order in $\nu$ that

$$
\bar{\eta}=B_{j 0}+C_{j 0}, \quad j=1, \ldots, K ; \quad \sum_{j=1}^{K} C_{j 0}=0 .
$$

We solve (6.18) and use (6.17) to eliminate $C_{j 0}$. This yields $\bar{\eta}=K^{-1} \sum_{j=1}^{K} B_{j 0}$, where $B_{j 0}$ satisfies

$$
\left(1+\frac{b_{0}}{\chi_{0 j}^{2}}\right) B_{j 0}-\frac{1}{K} \sum_{j=1}^{K} B_{j 0}=-\frac{2}{D_{0}} \int_{0}^{\infty} \rho w \Phi_{j} d \rho, \quad j=1, \ldots, K .
$$

Then, by substituting (6.19) into the equation (6.14a) for $\Phi_{j}$, we obtain $K$ coupled nonlocal eigenvalue problems. 
In matrix form this system can be written for $\Phi_{v} \equiv\left(\Phi_{1}, \ldots, \Phi_{K}\right)^{t}$ as

$$
\mathcal{L} \Phi_{v}-2 w^{2}\left(\frac{\int_{0}^{\infty} \rho w \mathcal{H} \Phi_{v} d \rho}{\int_{0}^{\infty} \rho w^{2} d \rho}\right)=\lambda \Phi_{v}, \quad 0 \leq \rho<\infty,
$$

where the matrix $\mathcal{H}$ is defined in terms of a diagonal matrix $\mathcal{D}$ and the vector $e=(1, \ldots, 1)^{t}$ by

$$
\mathcal{H} \equiv \mathcal{D}(I+\mathcal{D}-\mathcal{E})^{-1}, \quad \mathcal{E}=\frac{1}{K} e e^{t} ; \quad \mathcal{D}_{j j}=b_{0} / \chi_{0 j}^{2}, \quad j=1, \ldots, K
$$

By diagonalizing (6.20) we recover the following result of [54]:

Principal Result 6.1: Let $\varepsilon \rightarrow 0$ and suppose that $D=D_{0} / \nu \gg 1$, where $\nu=-1 / \log \varepsilon$ with $D_{0}=\mathcal{O}(1)$. Then, the stability of a $K$-spot solution is determined by the spectrum of the nonlocal eigenvalue problems

$$
\mathcal{L} \psi-2 w^{2} \kappa_{i} \frac{\int_{0}^{\infty} \rho w \psi d \rho}{\int_{0}^{\infty} \rho w^{2} d \rho}=\lambda \psi, \quad 0 \leq \rho<\infty ; \quad \psi \rightarrow 0 \quad \text { as } \quad \rho \rightarrow \infty,
$$

where $\kappa_{i}$ for $i=1, \ldots, K$ are the eigenvalues of the matrix $\mathcal{H}$ defined in (6.20 b). For a one-spot solution we have stability (i.e. $\operatorname{Re}(\lambda)<0$ ) for any value of $D_{0}=\mathcal{O}(1)$. Alternatively, a symmetric $K$-spot pattern with $K>1$, for which $\chi_{0 j}=\chi_{0}$ for $j=1, \ldots, K$, is stable if and only if $D_{0}<D_{0 K}$, where the threshold $D_{0 K}$ was given in (6.1).

To prove this result, we calculate for a symmetric $K$-spot pattern that the eigenvalues of $\mathcal{H}$ are simply

$$
\kappa_{i}=\frac{b_{0}}{\chi_{0}^{2}}\left[1+\frac{b_{0}}{\chi_{0}^{2}}\right]^{-1}, \quad i=1, \ldots, K-1, \quad \kappa_{K}=1 .
$$

The stability criterion of Theorem 5.1 of $[\mathbf{4 8}]$ and of $[\mathbf{5 2}]$ proves that $\operatorname{Re}(\lambda)<0$ if and only if $\kappa_{i}>1 / 2$ for $i=1, \ldots, K$ (see also Appendix B of [54]). Therefore, since $\kappa_{K}=1$, a one-spot solution is always stable. For $K>1$, we use (6.22) for $\kappa_{i}$ to conclude that a $K$-spot pattern is stable if and only if $\chi_{0} / \sqrt{b}_{0}<1$. Finally, by using $(6.12)$ for $\chi_{0}$ we obtain the threshold value $D_{0 K}$ of (6.1). In principle by using a higher order expansion for the solutions to the quasi-equilibrium problem (3.2) and (3.6) and of the eigenvalue problem (3.26) and (3.20), we should be able to calculate an $\mathcal{O}\left(\nu^{1 / 2}\right)$ correction term to the threshold $D_{0 K}$.

\section{Discussion}

We have developed a formal asymptotic analysis to derive a DAE system (3.6) and (3.17) describing the dynamics of a collection of spots for the Schnakenburg model (1.2). By studying the stability properties of a single spot numerically, we have formulated a criterion for the occurrence of spot-splitting behavior in (1.2) in terms of the solution to the DAE system. By solving this DAE system numerically, and analytically for special ring-type patterns of spots, we have favorably compared our asymptotic results for spot dynamics with full numerical solutions of (1.2).

There are several open problems for the Schnakenburg model that warrant further study. The first, and most important, such problem is to provide a rigorous mathematical theory to supplement the formal asymptotic analysis presented here. Motivated by the bistable phenomena discovered in $\S 5.2$ for certain ring-type patterns of spots in the unit disk, a second key open problem is to perform a detailed bifurcation study of the equilibria of the DAE system (3.6) and (3.17) in order to characterize all possible equilibrium spot-type patterns in an arbitrary domain and to investigate how these solution branches depend on the parameters. A third important open problem is to derive correction terms to the leading-order NLEP theory of $\S 6$ to determine thresholds for spot stability when $D \gg \mathcal{O}(1)$, and to numerically study the matrix problem of (3.28) governing the stability of the core solution to locally radially symmetric perturbations when $D=\mathcal{O}(1)$. A fourth open problem is to numerically calculate the solution branch 
that bifurcates off of the radially symmetric core solution at the critical value where a peanut-splitting instability is initiated.

With regards to more general systems, a key open problem is to investigate whether the asymptotic methodology developed here can be readily extended to other multi-component reaction-diffusion systems in the semi-strong limit of small diffusivity of only one of the components. Such related systems include the Gierer-Meinhardt and Gray-Scott models. More specifically, for which class of reaction-diffusion models can one guarantee that the primary instability of the core solution is to a peanut-splitting instability that initiates a spot-splitting event? For such systems it should be possible to derive related DAE systems governing the dynamics of a collection of spots and to formulate a criterion for the initiation of spot-splitting. In contrast to the Schakenburg model studied in this paper in a finite domain, it should be possible to analyze spot patterns for these other reaction-diffusion models on infinite domains provided that the relevant Green's function associated with the inhibitor concentration decays at infinity. Finally, it would also be interesting to develop a similar asymptotic methodology to analyze various bifurcations associated with localized spot patterns on slowly growing domains.

\section{Acknowledgements}

T. K. and M. J. W. were supported by NSERC (Canada). Prof. Juncheng Wei was partially supported by an Earmarked Grant from RGC of Hong Kong. We are grateful to Prof. P. Zegeling for allowing us to use his code VLUGR2 to calculate full numerical solutions of the Schnakenburg model.

\section{References}

[1] E. Anderson et al. LAPACK User's Guide: Third Edition, SIAM Publications (1999).

[2] U. Ascher, R. Christiansen, R. Russell, Collocation Software for Boundary Value ODE's, Math. Comp., 33, (1979), pp. 659-679.

[3] Y. A. Astrov, H. G. Purwins, Spontaneous Division of Dissipative Solitions in a Planar Gas-Discharge System with High Ohmic Electrode, Phys. Lett. A, 358, No. 5-6, (2006), pp. 404-408.

[4] Y. A. Astrov, H. G. Purwins, Plasma Spots in a Gas Discharge System: Birth, Scattering and Formation of Molecules, Phys. Lett. A, 283, No. 3-4, (2001), pp. 349-354.

[5] I. Barrass, E. J. Crampin, P. K. Maini, Mode Transitions in a Model Reaction-Diffusion System Driven by Domain Growth and Noise, Bull. Math. Biol., 68, (2006), pp. 981-995.

[6] J. G. Blom, R. A. Trompert, J. G. Verwer, Algorithm 758: VLUGR2: A Vectorizable Adaptive-Grid Solver for PDEs in 2D, ACM Trans. Math. Softw., 22, No. 3, (1996), pp. 302-328.

[7] M. Bode, A. W. Liehr, C. P. Schenk, H. G. Purwins, Interactions of Dissipative Solitons: Particle-Like Behavior of Localized Structures in a Three Component Reaction-Diffusion System, Physica D 161, No. 1-2, (2002), pp. 45-66.

[8] P. C. Bressloff, B. E. Earnshaw, M. J. Ward, Diffusion of Protein Receptors on a Cylindrical Dendritic Membrane with Partially Absorbing Traps, submitted, SIAM J. Appl. Math., (2007).

[9] X. Chen, M. Kowalczyk, Dynamics of an Interior Spike in the Gierer-Meinhardt System, SIAM J. Math. Anal., 33, No. 1, (2001), pp. 172-193.

[10] E. J. Crampin, E. Gaffney, P. K. Maini, Reaction and Diffusion on Growing Domains: Scenarios for Robust Pattern Formation, Bull. Math. Biol., 61, (1999), pp. 1093-1120.

[11] P. W. Davis, P. Blanchedeau, E. Dullos, P. De Kepper, Dividing Blobs, Chemical Flowers, and Patterned Islands in a Reaction-Diffusion System, J. Phys. Chem. A, 102, No. 43, (1998), pp. 8236-8244.

[12] A. Doelman, R. A. Gardner, T. J. Kaper, Stability Analysis of Singular Patterns in the 1D Gray-Scott Model: A Matched Asymptotics Approach, Physica D, 122, No. 1-4, (1998), pp. 1-36.

[13] A. Doelman, T. J. Kaper, L. A. Peletier, Homoclinic Bifurcations at the Onset of Pulse Self-Replication, J. Differential Equations, 231, No. 1, (2006), pp. 359-423.

[14] E. W, Dynamics of Vortices in Ginzburg Landau Theories with Applications to Superconductivity, Physica D, 77, No. 4, (1994), pp. 383-404. 
[15] S. Ei, Y. Nishiura, K. Ueda, $2^{n}$ Splitting or Edge Splitting?: A Manner of Splitting in Dissipative Systems, Japan J. Indust. Appl. Math., 18, (2001), pp. 181-205.

[16] S. Ei, M. Mimura, M. Nagayama, Pulse-Pulse Interaction in Reaction-Diffusion Systems, Physica D, 165, No. 3-4, (2002), pp. $176-198$.

[17] S. Ei, M. Mimura, M. Nagayama, Interacting Spots in Reaction-Diffusion Systems, Discrete Contin. Dyn. Sys., 14, No. 1, (2006), pp. 31-62.

[18] S. Ei, J. Wei, Dynamics of Metastable Localized Patterns and its Application to the Interaction of Spike Solutions for the Gierer-Meinhardt System in Two Space Dimensions, Japan J. Indust. Appl. Math., 19, No. 2, (2002), pp. $181-226$.

[19] E. A. Gaffney, N. A. M. Monk, Gene Expression Time Delays and Turing Pattern Formation Systems, Bull. Math. Biol., 68, No. 1, (2006), pp. 99-130.

[20] I. M. Gradshteyn, I. M. Ryzhik, Table of Integrals, Series, and Products, corrected and enlarged edition, pp. 36-41, 1980, Academic Press.

[21] S. Gueron, I. Shafrir, On a Discrete Variational Problem Involving Interacting Particles, SIAM J. Appl. Math., 60, No. 1, (1999), pp. 1-17.

[22] S. Gustafson, I. M. Sigal, Effective Dynamics of Magnetic Vortices, Adv. Math., 109, No. 2, (2006), pp. $448-498$.

[23] D. Iron, J. Wei, M. Winter, Stability Analysis of Turing Patterns Generated by the Schnakenburg Model, J. Math. Biol., 49, No. 4, (2004), pp. 358-390.

[24] T. Kolokolnikov, M. J. Ward, Reduced Wave Green's Functions and Their Effect on the Dynamics of a Spike for the Gierer-Meinhardt Model, European J. Appl. Math., 14, No. 5, (2003), pp. 513-545.

[25] T. Kolokolnikov, M. Ward, J. Wei, The Stability of Spike Equilibria in the One-Dimensional Gray-Scott Model: The Pulse-Splitting Regime, Physica D, 202, No. 3-4, (2005), pp. 258-293.

[26] T. Kolokolnikov, M. S. Titcombe, M. J. Ward, Optimizing the Fundamental Neumann Eigenvalue for the Laplacian in a Domain with Small Traps, European J. Appl. Math., 16, No. 2, (2005), pp. 161-200.

[27] T. Koloklonikov, W. Sun, M. J. Ward, J. Wei, The Stability of a Stripe for the Gierer-Meinhardt Model and the Effect of Saturation, SIAM J. Appl. Dyn. Sys., 5, No. 2, (2006), pp. 313-363.

[28] K. J. Lee, W. D. McCormick, J. E. Pearson, H. L. Swinney, Experimental Observation of Self-Replicating Spots in a Reaction-Diffusion System, Nature, 369, (1994), pp. 215-218.

[29] C. S. Lin, W. M. Ni, I. Takagi, Large Amplitude Stationary Solutions to a Chemotaxis System, J. Differential Equations, 72, (1988), pp. 1-27.

[30] A. Madzvamuse, A. J. Wathen, P. K. Maini, A Moving Grid Finite Element Method Applied to a Model Biological Pattern Generator, J. Comput. Physics, 190, (2003), pp. 478-500.

[31] A. Madzvamuse, P. K. Maini, A. J. Wathen, A Moving Grid Finite Element Method for the Simulation of Pattern Generation by Turing Models on Growing Domains, J. Sci. Computing, 24, No. 2, (2005), pp. $247-262$.

[32] S. L. Marshall, A Rapidly Convergent Modified Green's Function for Laplace's Equation in a Rectangular Domain, Proc. Roy. Soc. London A, 455, (1999), pp. 1739-1766.

[33] R. C. McCann, R. D. Hazlett, D. K. Babu, Highly Accurate Approximations of Green's and Neumann Functions on Rectangular Domains, Proc. Roy. Soc. London A, 457, (2001), pp. 767-772.

[34] C. Muratov, V. V. Osipov, Static Spike Autosolitons in the Gray-Scott Model, J. Phys. A: Math Gen. 33, (2000), pp. 88938916.

[35] C. Muratov, V. V. Osipov, Spike Autosolitons and Pattern Formation Scenarios in the Two-Dimensional Gray-Scott Model, Eur. Phys. J. B. 22, (2001), pp. 213-221.

[36] S. Nasuno, Dancing "Atoms" and "Molecules" of Luminous Gas-Discharge Spots, Chaos, 13, No. 3, (2003), pp. 1010-1013.

[37] Y. Nishiura, D. Ueyama, A Skeleton Structure of Self-Replicating Dynamics, Physica D, 130, No. 1-2, (1999), pp. 73-104.

[38] Y. Nishiura, D. Ueyama, Spatio-Temporal Chaos for the Gray-Scott Model, Physica D, 150, No. 3-4, (2001), pp. 137-162.

[39] J. E. Pearson, Complex Patterns in a Simple System, Science, 216, (1993), pp. 189-192.

[40] R. Straube, M. J. Ward, M. Falcke, Reaction Rate of Small Diffusing Moelecules on a Cylindrical Membrane, to appear, J. Stat. Phys., (2008), (29 pages).

[41] W. Sun, M. J. Ward, R. Russell, The Slow Dynamics of Two-Spike Solutions for the Gray-Scott and Gierer-Meinhardt Systems: Competition and Oscillatory Instabilities, SIAM J. Appl. Dyn. Sys., 4, No. 4, (2005), pp. $904-953$.

[42] M. S. Titcombe, M. J. Ward, An Asymptotic Study of Oxygen Transport from Multiple Capillaries to Skeletal Muscle Tissue, SIAM J. Appl. Math., 60, No. 5, (2000), pp. 1767-1788.

[43] D. Ueyama, Dynamics of Self-Replicating Patterns in the One-Dimensional Gray-Scott Model, Hokkaido Math J., 28, No. 1, (1999), pp. 175-210.

[44] V. K. Vanag, I. R. Epstein, Localized Patterns in Reaction-Diffusion Systems, Chaos 17, No. 3, 037110, (2007).

[45] M. J. Ward, W. D. Henshaw, J. Keller, Summing Logarithmic Expansions for Singularly Perturbed Eigenvalue Problems, SIAM J. Appl. Math, 53, No. 3, (1993), pp. 799-828.

[46] M. J. Ward, D. McInerney, P. Houston, D. Gavaghan, P. Maini, The Dynamics and Pinning of a Spike for a ReactionDiffusion System, SIAM J. Appl. Math., 62, No. 4, (2002), pp. 1297-1328. 
[47] M. J. Ward, J. Wei, The Existence and Stability of Asymmetric Spike Patterns for the Schnakenburg Model, Studies in Appl. Math., 109, No. 3, (2002), pp. 229-264.

[48] J. Wei, Existence, Stability, and Metastability of Point Condensation Patterns Generated by the Gray-Scott System, Nonlinearity, 12, (1999), pp. 593-616.

[49] J. Wei, M. Winter, Spikes for the Two-Dimensional Gierer-Meinhardt System: the Weak Coupling Case, J. Nonlinear Sci., 11, No. 6, (2001), pp. 415-458.

[50] J. Wei, M. Winter, Spikes for the Two-Dimensional Gierer-Meinhardt System: the Strong Coupling Case, J. Differential Equations, 178, No. 2, (2002), pp. 478-518.

[51] J. Wei, Pattern Formations in Two-Dimensional Gray-Scott Model: Existence of Single-Spot Solutions and Their Stability, Physica D, 148, No. 1-2, (2001), pp. 20-48.

[52] J. Wei, M. Winter, Existence and Stability of Multiple Spot Solutions for the Gray-Scott Model in $\mathbb{R}^{2}$, Physica D, 176, No. 3-4, (2003), pp. 147-180.

[53] J. Wei, M. Winter, Asymmetric Spotty Patterns for the Gray-Scott Model in $R^{2}$, Studies in Appl. Math., 110, No. 1, (2003), pp. 63-102.

[54] J. Wei, M. Winter, Stationary Multiple Spots for Reaction-Diffusion Systems, to appear, J. Math. Biology, (2007). 